\title{
Change Is the only constant
}

Citation for published version (APA):

Liu, Y. (2021). Change Is the only constant: the dynamic nature of attentional bias for food. [Doctoral Thesis, Maastricht University]. Maastricht University. https://doi.org/10.26481/dis.20211122yl

Document status and date:

Published: 01/01/2021

DOI:

10.26481/dis.20211122yl

Document Version:

Publisher's PDF, also known as Version of record

\section{Please check the document version of this publication:}

- A submitted manuscript is the version of the article upon submission and before peer-review. There can be important differences between the submitted version and the official published version of record.

People interested in the research are advised to contact the author for the final version of the publication, or visit the DOI to the publisher's website.

- The final author version and the galley proof are versions of the publication after peer review.

- The final published version features the final layout of the paper including the volume, issue and page numbers.

Link to publication

\footnotetext{
General rights rights.

- You may freely distribute the URL identifying the publication in the public portal. please follow below link for the End User Agreement:

www.umlib.nl/taverne-license

Take down policy

If you believe that this document breaches copyright please contact us at:

repository@maastrichtuniversity.nl

providing details and we will investigate your claim.
}

Copyright and moral rights for the publications made accessible in the public portal are retained by the authors and/or other copyright owners and it is a condition of accessing publications that users recognise and abide by the legal requirements associated with these

- Users may download and print one copy of any publication from the public portal for the purpose of private study or research.

- You may not further distribute the material or use it for any profit-making activity or commercial gain

If the publication is distributed under the terms of Article $25 \mathrm{fa}$ of the Dutch Copyright Act, indicated by the "Taverne" license above, 
Change Is the Only Constant:

The Dynamic Nature of Attentional Bias for Food 
ISBN: 978-94-6423-457-2

Print: Gildeprint | www.gildeprint.nl

(C) Copyright 2021: Yu Liu

All rights reserved. No part of this publication may be reproduced, stored in a retrieval system, or mechanical, by photocopying, recording, or otherwise, without the prior written permission of the author. 


\section{Change Is the Only Constant: \\ The Dynamic Nature of Attentional Bias for Food}

\section{Dissertation}

to obtain the degree of Doctor at Maastricht University, on the authority of the Rector Magnificus, Prof.dr. Rianne M. Letschert in accordance with the decision of the Board of Deans, to be defended in public on Monday, 22 November, 2021 at 16:00

by

Yu Liu 


\section{Promotors}

Prof. Dr. C. Nederkoorn

Prof. Dr. A.J. Roefs

\section{Assessment Committee}

Prof. Dr. M.L. Peters (chair)

Prof. Dr. H.P. Otgaar

Prof. Dr. I.H.A. Franken (Erasmus University Rotterdam)

Dr. N.C. Jonker (University of Groningen)

Prof. Dr. P.E.H.M. Muris 


\section{Table of contents}

page

$\begin{array}{lll}\text { Chapter } 1 & \text { General introduction } & 7\end{array}$

Chapter 2 Dynamics of attentional bias for food in adults, children, 23 and restrained eaters

Chapter 3 Food palatability directs our eyes across contexts

Chapter 4 Dynamics of attentional bias for food in Dutch and 65 Chinese children and the role of executive control

Chapter 5 Fluctuations in attentional bias for food and the role of executive control

Chapter 6 Attentional capture by food distractors: the role of perceptual load

Chapter 7 General discussion

Valorization addendum

References

Acknowledge

Curriculum Vitae 


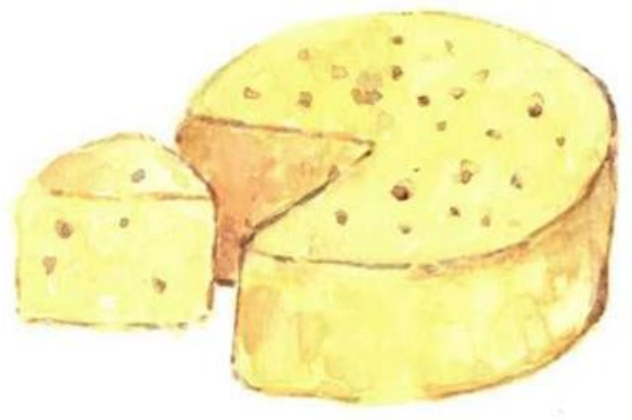


Chapter 1

General Introduction 


\section{Obesity epidemic, problematic food-rich environment, and attention}

Worldwide, the prevalence of obesity nearly tripled between 1975 and 2016 (World Health Organization, 2020), and forecasting models estimate that by 2030, up to $57.8 \%$ of the world's adult population (3.3 billion people) could be either overweight or obese (Kelly, Yang, Chen, Reynolds, \& He, 2005). Obesity has caused serious consequences, such as poorer physical and mental health and high economic burden (e.g., Kyrgiou et al., 2017; Smith, Campbell, \& Trollor, 2011; Tremmel, Gerdtham, Nilsson, \& Saha, 2017). Given the prevalence and detrimental effects of overweight and obesity, it is critical to identify and target the underlying mechanisms of overweight and obesity.

It is well known that the fundamental cause of overweight and obesity is an energy imbalance between calorie consumption and calorie expenditure. Nowadays, we live in an “obesogenic environment" (e.g., Hall, 2018; Lake, 2018), in which there is an abundance of high-calorie palatable foods, which are aggressively marketed and advertised (Brownell, 2002). This food-rich environment has promoted overeating in several ways, like increased affordability and accessibility of non-perishable, highly processed, and pre-packaged foods (Wright \& Aronne, 2012). People are therefore frequently exposed to high-calorie palatable foods or food-related stimuli, which might cause increased attention for food and subsequent food intake.

Attention to food might therefore play a role in the development and maintenance of overweight/obesity. From an evolutionary perspective, food is vital for people. It does not only provide nutrients needed to survive but is also a great source of reward. Searching for high-calorie foods is therefore an important function of the human brain (Drewnowski, 1997a, 1997b). As a consequence, it is argued that high-calorie palatable foods are generally salient and people are naturally attracted by high-calorie palatable foods (e.g., Davids et al., 2010; McSorley, Morriss, \& van Reekum, 2017), although empirical evidence is not entirely consistent (e.g., Hagan, Alasmar, Exum, Chinn, \& Forbush, 2020; Roefs, Houben, \& Werthmann, 2015). Incentive salience theory (Berridge, 2009; Berridge, Ho, Richard, \& DiFeliceantonio, 2010, Robinson \& Berridge, 1993) provides a cognitive model to explain the underlying mechanism of biased attention to food: The consumption of palatable foods leads to dopamine release in reward-related brain regions, which makes rewarding foods 
more salient. In addition, when food-related stimuli (i.e., viewing of (pictures) palatable foods) are repeatedly paired with actual food intake through Pavlovian conditioning, these stimuli can also gain incentive properties and become attention grabbing. It has been found that cue-conditioned overeating is quickly learned (Boggiano, Dorsey, Thomas, \& Murdaugh, 2009). Indeed, high-calorie palatable foods are very attractive, they are omnipresent in the food-rich environment, and we are prone to learn associations between food-related stimuli and hedonic eating in daily life. These factors may all contribute to problematic eating and weight gain.

\section{The stable trait-like view of attentional bias for food: increasing conflicts}

The "obesogenic environment" (e.g., Hall, 2018; Lake, 2018) does not influence everyone equally: some people struggle with increased food intake and weight problems, whereas others do not. A logical hypothesis seems that heightened attention to high-calorie palatable foods is related to increased food intake, higher BMI, and more struggles with weight. Field et al. (2016) stated that this view derives from incentive salience theory (Robinson \& Berridge, 1993) and was further developed in subsequent research. For example, hedonic reaction and reward-related motivation (reflected by a biased attention for food) are mostly "go" systems, of which satiety can merely tone down the intensity instead of completely "stop" them (Berridge, 2009). Overweight/obese individuals are linked to an enhanced attention towards high-calorie foods, which is associated with subjective ratings of food craving (Nijs \& Franken, 2012). It was proposed that attentional bias (AB) for food theoretically predicts obesity (Hendrikse et al., 2015). These statements all imply that an AB for food is a stable feature, which is more prominent in people with overweight/obesity and which can even cause obesity. However, there are several problems with this hypothesis, resulting from both empirical findings and from a theoretical perspective, which will be explained below.

\section{Empirical conflict}

The empirical conflict is clearly revealed by reviews and meta-analyses of $\mathrm{AB}$ for food in individuals with overweight/obesity, restrained eaters, and healthy-weight people (e.g., Doolan, Breslin, Hanna, \& Gallagher, 2015; Hagan et al., 2020; Werthmann, Jansen, \& Roefs, 2015). Some studies indeed support the hypothesis that people with overweight/obesity or 
restrained eaters show more biased attention to food as compared to a control group. For example, one study found that obese participants showed a reaction time (RT)-based AB for food in a food dot-probe task, whereas normal-weight participants did not show a significant food-relevant AB (Kemps, Tiggemann, \& Hollitt, 2014). Restrained eaters instead of unrestrained eaters responded faster to food stimuli than neutral stimuli in a flanker task (Meule, Vögele, \& Kübler, 2012). Using a word food Stroop task, another study reported that only children with obesity but not children with normal-weight were slower in naming the color of food words as compared to control words (Braet \& Crombez, 2003). In a Stroop task with a record of EEG activity, overweight women showed increased early attentional processes (increased right parietal P200 amplitude) and obese women showed increased maintained attentional processes (shortened right parietal P300 latency) of food stimuli as compared to normal-weight participants, whereas there was no group difference on the RTbased Stroop interference score (Hume, Howells, Rauch, Kroff, \& Lambert, 2015). To sum up, these studies suggest that people with overweight/obesity and restrained eaters show an increased $\mathrm{AB}$ for palatable foods as compared to control groups. However, it should be noted that it is quite debatable if the Stroop effect reflects $A B$ because it is unclear whether increased interference reflects attention towards or away from the stimuli (e.g., McNally, 2019; a more detailed explanation is presented on page 15).

Partly consistent with the stable trait-like view of $\mathrm{AB}$ for food, it was reported that in a food dot-probe task with eye-tracker, compared to healthy-weight participants, overweight/obese participants only biased their attention more to high-calorie food stimuli in early attentional processes (first fixation direction), but not in late attentional processes (total gaze duration), or RT-based AB for food (Werthmann et al., 2011). Another study also found that people with obesity allocated more early attention to food stimuli than to neutral stimuli (P200 component) (Nijs, Franken, \& Muris, 2010). However, all participants biased their attention to food words in late attentional processes (P300 component) and RT-based AB for food in a food Stroop task, with no significant group differences (Nijs, Franken, \& Muris, 2008). Moreover, results from a food dot-probe task showed an enhanced early attention towards food stimuli (stimuli were displayed for $100 \mathrm{~ms}$ ) in overweight/obese versus normalweight individuals, but only in a hungry state (Nijs, Muris, Euser, \& Franken, 2010). The P300 amplitude from this study showed that in the satiated state, AB to food was marginally 
larger in overweight/obese than in normal-weight women, whereas in the hungry state, AB to food was significantly larger in normal-weight than in overweight/obese participants. Taken together, these findings consistently revealed an early attention approach to food in people with overweight or obesity, whereas results of the late attention component were mixed. In addition, people's AB for food stimuli seems to be affected by their hunger level and the adopted paradigm to assess $\mathrm{AB}$ for food.

Contrary to the stable trait-like view of $\mathrm{AB}$ for food, other empirical studies found that $\mathrm{BMI}$ and dietary restraint were not significantly related to $\mathrm{AB}$ for food stimuli or were even negatively related to $\mathrm{AB}$ for food stimuli. Using a food dot-probe task with eye-tracker, one study found that all female participants expressed both greater RT-based and eyemovement based (gaze direction bias and total gaze duration bias) visual attention towards high-calorie food stimuli as compared to low-calorie food stimuli regardless of BMI status (Doolan, Breslin, Hanna, Murphy, \& Gallagher, 2014). Similarly, the eye-movement data from another study also revealed a biased gaze direction and total gaze duration to food stimuli in all participants regardless of weight and hunger status (Nijs, Muris et al., 2010). Another study focused on dietary restraint and they found that both restrained eaters and unrestrained eaters showed an RT-based and eye-movement-based AB for food stimuli in comparison to neutral stimuli (Werthmann et al., 2013). In addition, as compared to healthyweight participants, overweight/obese participants showed an attention away from food stimuli when paired stimuli presented either $200 \mathrm{~ms}$ or $2000 \mathrm{~ms}$ in a food dot-probe task (Fang et al., 2019). Merely low restrained eaters but not high restrained eaters marginally significantly biased their attention towards food stimuli in a food dot-probe task and significantly biased their attention towards food stimuli in a word food-Stroop task (Nathan et al., 2012). The above findings suggest that people with overweight/obesity and restrained eaters could either show an equal $A B$ to food stimuli or show a less $A B$ to food stimuli as compared to their respective control group.

Taken together, empirical evidence does not consistently support that $\mathrm{AB}$ for food is a stable trait-like feature of people with a high BMI or dietary restraint. On the contrary, the studies discussed above suggest that everyone, not specifically people with overweight/obesity or restrained eaters, may have an $\mathrm{AB}$ for food, but even this is not consistently observed. In addition, selective and sustained attention to food may be 
influenced by other factors, such as, which attentional subcomponent the study assessed (early versus late), hunger level, and the adopted paradigm to assess AB for food.

\section{Theoretical conflict}

Two factors that may affect $\mathrm{AB}$ for food have been largely ignored in research on $\mathrm{AB}$ for food so far. First, food is assumed to be only a source of reward, but high-calorie foods are also associated with weight gain. Second, it is mostly assumed that AB for food is stable over time, whereas it might actually be fluctuating. In addition, AB for food may depend on participants' executive control and attentional capacity.

First, palatable foods are not only a source of reward but are also a source of weight/health concerns (double-faceted nature of food, Roefs, Fransen, \& Jansen, 2018). Palatable foods can therefore also be seen as 'forbidden', wrong items that should be avoided or that even evoke fear (Miguet et al., 2018). This double-faceted nature of food is especially true in the Western "obesogenic environment" (e.g., Hall, 2018; Lake, 2018), in which not only high-calorie palatable foods are available almost everywhere, but also the popular thinideal body image, which is portrayed everywhere in the media (Johnson, Tobin, \& Steinberg, 1989). The assumption that palatable foods are always rewarding stimuli seems therefore an incorrect simplification.

The second assumption, that attention is a stable trait, seems also incorrect. When considering attention, it can be involuntarily captured by the salient stimuli (bottom-up). However, attention can also be voluntarily driven to stimuli with high priority to the observer (top-down) (Wolfe, 1994). In line with the double-faceted nature of food, appetitive motivation cannot be expected to be stable, but it is likely to fluctuate between food enjoyment and weight/health concerns. Correspondingly, attention allocation for food is not only automatically directed by the salient nature of food stimuli, if it is indeed salient, but also voluntarily directed by momentary food-related goals (top-down process) (Werthmann, Jansen, \& Roefs, 2015). That is, AB for food is also a reflection of the current appetitive motivation which reciprocally guides eating behavior through attention. Previous studies provide evidence to support this hypothesis. For example, in an online food choice task, health/diet-related banners were presented on the top of choice screens, which increased total lingering times on low-calorie products (van der Laan, Papies, Hooge, \& Smeets, 2017). 
Another study manipulated mindset (hedonic versus health), and found that an induced health mindset decreased RT-based AB for high-calorie food in participants with higher dietary restraint scores (Werthmann, Jansen, \& Roefs, 2016). In addition, participants who indicated that they did not permit themselves to eat chocolate in a so-called taste test that was part of the experiment showed significantly less craving and reduced total dwell time for chocolate stimuli than participants who permitted themselves to eat chocolate (Werthmann, Roefs, Nederkoorn, \& Jansen, 2013). Moreover, it was found that participants' total fixation duration was positively associated with both the amount of food eaten and the energy intake within a food category (Pedersen et al., 2021). So, previous studies may suggest that AB for food more likely fluctuates along with within-subject appetitive motivational states instead of more stable between-subject weight status (see Field et al., 2016 and Hardman et al., 2020 for a detailed review).

Building on this research, in the current dissertation it is hypothesized that such conflicting motivations can be expected to be larger for people who are more sensitive to both food-related reward and weight/health concerns, that is, people with overweight/obesity and high-restrained eaters (Stice, Spoor, Ng, \& Zald, 2009; Ahern, Field, Yokum, Bohon, \& Stice, 2010). In summary, if appetitive motivation fluctuates over time, AB for food, as a reflection of inner appetitive motivation, is also expected to fluctuate, and is unlikely to be a stable trait-like feature of these susceptible groups.

Executive control might affect the degree of AB fluctuation. First, it should be noted that food reward and weight/health concerns are two competing goals. Executive control is a top-down process that involves solving conflicting mindsets and helps with the pursuit of a goal (Engle, 2018). When there is a conflict between a long-term health goal and a short-term goal to enjoy palatable food, executive control is needed to inhibit impulsively reacting to rewarding foods and to generate a more considered response (Diamond, 2013). When people are high in executive control, the long-term goal to eat healthily might prevail, whereas the more impulsive goal to enjoy tasty food might dominate when executive control is low (e.g., Lapenta, Di Sierve, de Macedo, Fregni, \& Boggio, 2014; Lopez, Hofmann, Wagner, Kelley, $\&$ Heatherton, 2014; Lowe, Hall, \& Staines, 2014). In addition, executive control is also vital for sustained attention and task performance (Diamond, 2013). Therefore, executive control 
might also influence whether food-related stimuli are attended to or not and the extent of fluctuations in $\mathrm{AB}$ for food.

Finally, attentional capacity might play a role in the attentional capture by food as well. More specifically, our surroundings usually are filled with too much information, so that we cannot notice everything, because attention has limited capacity (Driver, 2001). Perceptually selecting the most relevant stimuli while filtering out distractors is the core function of our attention (Katsuki \& Constantinidis, 2014). Attention capacity can affect the filtering of information, and therefore may affect the attentional capture by food. Based on the limited capacity of attention, the perceptual load theory (Lavie, Hirst, De Fockert, \& Viding, 2004) proposed that increasing perceptual load in the relevant-task would leave no capacity to encode task-irrelevant distractors (e.g., Lavie, Lin, Zokaei, \& Thoma, 2009; Gupta, Hur, \& Lavie, 2016), unless the distractors were meaningful and familiar, for example when they are famous faces (e.g., Lavie, Ro, \& Russell, 2003; Ro, Friggel, \& Lavie, 2009; He \& Chen, 2010). Thus, the perceptual load theory suggests that $A B$ for food might also be influenced by the task load and available attentional capacity, although it is possible that food is so salient that it can escape a high perceptual load and still attracts attention.

In summary, it seems unjustified to assume that food is merely a source of reward, and instead, high-calorie foods can be rewarding, but can also elicit weight/health concerns. As a consequence, people with a high BMI and dietary restraint may frequently struggle with conflicting goals, and attention to food could alternate between approach and avoidance over time. In addition, whether food-related stimuli are attended to or not and the extent of fluctuations in attention might depend on executive control. Finally, perceptual capacity might also influence attentional capture by food. All in all, these factors all could affect individuals' $\mathrm{AB}$ for food and therefore, lead to fluctuations in $\mathrm{AB}$ food. These fluctuations in $\mathrm{AB}$ for food could happen across studies, but also within one study, within one participant. So, to correctly understand individuals' $\mathrm{AB}$ for food, on the one hand, it is important to manipulate the factors that we proposed (appetitive motivation, executive control, and attentional capacity) to test their influence on $\mathrm{AB}$ for food, and on the other hand, it is important to measure fluctuations in $\mathrm{AB}$ for food within one study within one participant. Next, it is also important to combine these two research goals and test how the various factors influence fluctuations in $\mathrm{AB}$ for food. Finally, as we stated before, empirical results seem to Page | 14 
suggest that the paradigm adopted to measure $A B$ for food may also affect $A B$ for food. So, it is important to adopt an appropriate paradigm to assess AB for food.

\section{Visual attention assessment and mean attentional bias for food}

Two paradigms have been most commonly used in previous studies to assess $\mathrm{AB}$ for food: food-Stroop task (Overduin, Jansen \& Louwerse, 1995) and food dot-probe task (MacLeod, Mathews, \& Tata, 1986). The food-Stroop task is used to measure the RT-based interference effect caused by food versus control stimuli. More specifically, colored food and non-food words are displayed in the task, and participants are required to respond to the color of the words as quickly and accurately as possible while ignoring the meaning of the words. The AB score for food (average interference from food) is computed by subtracting the mean RT on non-food trials from the mean RT on food trials. A positive score is taken to reflect attention towards food, whereas a negative score is taken to reflect attention away from food. However, in fact, both attention towards and avoidance of food can slow down color-naming (Williams, Mathews, \& MacLeod, 1996). That is, it is uncertain if a slower response latency reflects increased or decreased attention for the food word stimulus. In addition, the foodStroop task might measure the ability to inhibit interference from the meaning of food words (attention control), which is more likely to reflect late attentional processes (Phaf \& Kan, 2007). It is therefore hard to know which attentional mechanism the interference score, as obtained in this paradigm, reflects.

In the food dot-probe task, pairs of food and nonfood words or pictures are presented on the screen and followed by a probe, which is presented in the location of either the food (congruent trial) or nonfood stimulus (incongruent trial). Participants are required to indicate the location of the probe as quickly and accurately as possible. The AB score for food is calculated by subtracting the mean RT on congruent trials from the mean RT on incongruent trials. A faster response on congruent trials (positive $\mathrm{AB}$ score) is taken to reflect an $\mathrm{AB}$ toward food. One benefit of the food dot-probe task is that it is possible to track different attentional processes, early versus late attention processes, either by varying presentation times of the picture stimuli or by concurrently measuring eye movements. Previous research suggests that stimulus presentation times below $500 \mathrm{~ms}$ should be used to assess an early attention component, whereas presentation times above $500 \mathrm{~ms}$ should be used to assess a 
late attention component (Field \& Cox, 2008). The food dot-probe task with concurrent measurement of eye-movements directly reflects eye-movement trajectories and gives realtime insight into people's attention to food within one trial. So, compared to the food-Stroop task, the food dot-probe task has some advantages that it can measure attentional allocation and in addition, it possible to capture different attentional processes. The current dissertation, therefore, adopted the food dot-probe task to assess participants' AB for food.

\section{Variability of attentional bias for food}

Considering that $\mathrm{AB}$ may not be a fixed individual difference variable, but may fluctuate continuously over time, two research groups recently proposed relative new computing methods to capture the fluctuations of $\mathrm{AB}$ for emotion-related stimuli in the dot-probe task (Iacoviello et al., 2014; Zvielli, Bernstein, \& Koster, 2015). Although both methods aim to investigate within-subject fluctuations in $\mathrm{AB}$ for critical stimuli rather than between-group differences, these two methodologies applied different approaches to calculate $\mathrm{AB}$ variability (see below for further explanations). Note that these two approaches so far have only been validated in emotion-related research (e.g., Naim et al., 2015; Clarke et al., 2020).

\section{Attention bias variability}

Lacoviello and colleagues (2014) argued that intra-individual variability of AB for threatrelated stimuli may not only explain the inconsistent findings of $\mathrm{AB}$ for threat-related stimuli in people with posttraumatic stress disorder (PTSD), but it could also be a better predictor of impaired attention control in PTSD, as compared to mean AB score. To quantify variability in $\mathrm{AB}$, attention bias variability (ABV) was calculated (Iacoviello et al., 2014). More specifically, first, all dot-probe trials were split into several bins with the same number of sequential trials in each bin (for example 20 sequential trials in a bin was adopted in Lacoviello et al. (2014)'s seminal study), and then mean AB scores were calculated for each bin. $\mathrm{ABV}$ is the standard deviation $(S D)$ of the mean $\mathrm{AB}$ scores across the bins, divided by each individual's mean RT across trials (to correct for variance in RTs). From the ABV formula, it can be seen that ABV quantifies the intra-individual variability of bias in an intrasession manner, rather than quantifying trial-by-trial variability. This index has been validated in several studies. 
For example, when assessing $\mathrm{AB}$ for threat-related stimuli, the greatest $\mathrm{ABV}$ was found in the PTSD group as compared to both a trauma-exposed without PTSD group and a healthy control group (Iacoviello et al., 2014; Naim et al., 2015) and ABV was consistently related to PTSD symptom severity (Swick \& Ashley, 2017). In addition, ABV instead of mean $\mathrm{AB}$ successfully differentiated combat-exposed and nonexposed soldiers during deployment but not before deployment (Iacoviello et al., 2014). Another study investigated the relationship between $\mathrm{AB}$ for painful/happy facial expressions and post-traumatic stress symptoms (PTSS). It was found that veterans with chronic pain showed significantly enhanced fluctuations in $\mathrm{AB}$ for both painful and happy facial expressions as compared to the control group, and fluctuations in $\mathrm{AB}$ for both painful and happy facial expressions was correlated with PTSS among all participants (Mazidi, Vig, Ranjbar, Ebrahimi, \& Khatibi, 2019). In sum, these findings suggest that ABV is a valid index to quantify the degree of some emotion-related symptoms and as compared to mean $\mathrm{AB}$ scores, $\mathrm{ABV}$ is more related to emotion-related symptoms.

\section{Trial-level bias scores}

Another computing method directly targets trial-level-bias scores (TL-BS), reflecting the time-series of $\mathrm{AB}$, and represents fluctuations of $\mathrm{AB}$ in consecutive bias scores (Zvielli et al., 2015). TL-BS makes the most use of all trials to create a time-series of $A B$ scores over the course of the task. More specifically, it firstly matches each congruent trial with an in-time closest incongruent trial and keeps the inter-trial interval between matched trials within five trials away. Then, a long series of bias scores are produced by subtracting the RT of each of the congruent trial from its paired incongruent trial. The sequential bias scores indicate how attention is allocated between critical and non-critical stimuli over time, which can be presented in a plot to visually inspect the dynamic nature of AB fluctuations (an example can be seen in Figure 1, chapter 2, page 30). In addition, TL-BS variability can be computed to quantify the degree of fluctuations between attention toward and away from critical stimuli. This index is computed using the sum of absolute distances between sequential bias scores divided by the total number of bias scores.

TL-BS variability not only demonstrated a higher split-half reliability than mean bias scores (Caudek, Ceccarini, \& Sica, 2017; Zvielli et al., 2015) but also has been validated 
in several domains. For example, individuals with spider phobia and individuals with depression exhibited significantly greater TL-BS variability but not larger mean AB scores, as compared to healthy control participants (Zvielli, Vrijsen, Koster, \& Bernstein, 2016; Zvielli et al., 2015). TL-BS variability was related to anxiety symptoms (Clarke et al., 2020) and it was significantly reduced after adults with social anxiety disorder accepted cognitivebehavioral therapy (CBT) (Bardeen, Daniel, Hinnant, \& Orcutt, 2017). All these empirical studies suggest that TL-BS variability can trustfully index the severity of some emotionrelated symptoms.

Comparing the ABV and TL-BS formula, it seems that TL-BS can better express momentary changes of $\mathrm{AB}$ for critical stimuli than $\mathrm{ABV}$. In addition, TL-BS provides more information, because it not only uses TL-BS variability to quantify the extent of attention towards and away from critical stimuli but also presents momentary AB bias scores in a plot to show the dynamic nature of $\mathrm{AB}$ for critical stimuli. Considering the benefits of TL-BS, this computing method was adopted to express the dynamic nature of $\mathrm{AB}$ for food in the current dissertation.

\section{Moving forward with caution}

Kruijt, Field, and Fox (2016) argued that one should be cautious when applying these two new computing methods. They manipulated the mean RT, SD in RT, and the magnitude of $\mathrm{AB}$ in randomly generated datasets to assess the properties of $\mathrm{ABV}$ and TL-BS indices. It was found that although both ABV and TL-BS variability were sensitive to the extent of fluctuations in $\mathrm{AB}$ for critical stimuli, they were also sensitive to the extent of fluctuations in RTs. So, they concluded that it is impossible to know whether ABV and TL-BS variability reflect fluctuations in $\mathrm{AB}$ or in general responding (reaction times). Therefore, $\mathrm{AB}$ variability may be a by-product of executive control or other measurement errors instead of bias variability. It should be noted that the data used in Kruijt et al. (2016) was generated by a computer and was normally distributed, which is different from real experimental data. One anonymous reviewer of Kruijt et al. (2016) argued that such normally distributed data allowed them to treat RT and standard deviation as independent whereas they are not in typically RT data. In addition, even if ABV and TL-BS variability were sensitive to the extent of fluctuations in RTs, it does not mean that they cannot predict unique variance in symptoms, 
and it is still unclear why ABV and TL-BS variability are related to some emotion-related symptoms so far.

In summary, it is too early to draw conclusions about the usefulness of these two methods at this moment. More empirical studies are worthwhile to conduct in broad domains to validate $\mathrm{ABV}$ and TL-BS to resolve the raised concern, and to detect which cognitive process they capture. In short, both methodologies seem promising to help explain and converge the inconsistent results of $\mathrm{AB}$ for food in individuals with overweight/obesity or restraint eaters. However, the application in the food domain still needs to be tested. In addition, it is also important to address the concern raised by Kruijt et al. (2016). For example, in the food domain, we can use real experimental data to further test whether TL-BS variability for food can significantly explain more variance after controlling for general reaction times and whether BMI is specifically related to TL-BS variability for food instead of for other nonfood stimuli. Finally, the underlying mechanisms behind increased TL-BS variability for food and enhanced BMI, if they are indeed significantly related to each other, are worthwhile to further explore.

\section{Outline and hypothesis of the current dissertation}

In sum, people's attention sometimes is attracted by palatable foods. However, the attentional capture by food might not be stable and $\mathrm{AB}$ for food appears not to be a trait-like characteristic of certain groups, such as people with overweight. Instead, it is proposed that $\mathrm{AB}$ for food is a dynamic process and depends on many factors, such as food-related motivations, executive control ability, and attentional capacity. The fluctuations in AB for food could occur both across studies and within one study within one participant, which could explain the conflicting results on $\mathrm{AB}$ for food in the literature. As fluctuations in $\mathrm{AB}$ for food have rarely been investigated, the main aim of the current dissertation is to explore this dynamic nature of $\mathrm{AB}$ for food. Therefore, we apply the food dot-probe task while concurrently measuring eye-movements, and use the TL-BS computing method to measure and quantify the fluctuations in $\mathrm{AB}$ for food. In addition, we study whether food captures attention more than neutral nonfood stimuli and investigate the role of attentional capacity on the attentional capture by food. Our work mainly focuses on the following four research goals. 
First, this dissertation tested the relationship between mean $\mathrm{AB}$ for food and BMI/dietary restraint in Chapters 2 to 5. Although the overarching aim of the current dissertation is to investigate the dynamic nature of $\mathrm{AB}$ for food, to verify there is indeed no consistent $\mathrm{AB}$ for food in people with a high $\mathrm{BMI}$ or dietary restraint, as it was suggested by previous research (e.g., Nijs, Muris et al., 2010; Hagan et al., 2020; Roefs et al., 2015), mean $\mathrm{AB}$ for food was also investigated. In addition, measuring mean $\mathrm{AB}$ scores also benefits to test whether TL-BS variability food has any added value to BMI/dietary restraint after controlling for mean $\mathrm{AB}$ for food.

Second, we explored the intra-individual fluctuations in $\mathrm{AB}$ for food across participants with Western and Eastern backgrounds (Chapters 2 to 5). Because there was no previous study investigating the dynamic nature of $\mathrm{AB}$ for food within one study, to have a first impression about it, we adopted the TL-BS method (Zvielli et al., 2015) to reanalyze RT-based and eye movement-based (dwell time bias) variability of AB for food from three published earlier studies (Werthmann et al., 2015; Werthmann et al., 2011, 2013) in Chapter 2. The target groups in these studies were overweight/obese adults, obese children, and healthy-weight restrained eaters. So, we could assess the relationship between TL-BS variability for food and BMI, and the relationship between TL-BS variability for food and dietary restraint. In addition, to broadly test the intra-individual fluctuations in AB for food, in Chapter 4, we assessed TL-BS variability for food in both Western (Dutch children) and Eastern (Chinese children) groups. Moreover, studies described in Chapters 3 and 5 also tested the second research question, along with additional research questions. It was assumed that TL-BS variability for food would be positively related to BMI and dietary restraint across participants.

As a third research goal, we tried to reveal the underlying mechanism behind the possibly significant and positive association between TL-BS variability for food and BMI (Chapters 3 to 5). As it was discussed before, we hypothesized that two factors may lead to the significant and positive association between TL-BS variability for food and BMI: appetitive motivation and executive control. In Chapter 3, we tested the influence of different appetitive motivations on TL-BS variability for food. Theoretically, fluctuations in AB for food might reflect the alternating focus on food enjoyment versus weight/health concerns when confronted with palatable food. So, it was expected that people, especially restrained 
eaters who frequently experience conflict between food enjoyment and weight concerns (Stroebe, van Koningsbruggen, Papies, \& Aarts, 2013), might have more and more consistent $\mathrm{AB}$ for food when one of their food-related goals was experimentally activated (either hedonic or heath goal) as compared to in a neutral state. To examine our hypotheses, we successively primed female participants with a hedonic, healthy, and neutral context (in balanced order) in a blocked fashion embedded in a food dot-probe task with concurrent measurement of eye-movements, and examined the effect of context priming on both average and TL-BS variability for food and the role of dietary restraint.

Another factor that was hypothesized to explain the enhanced intra-individual fluctuations in $\mathrm{AB}$ for food in people with a high BMI is executive control, and this was tested in Chapters 4 and 5. Studies included in Chapter 4 tested the moderating effect of attention control, measured by the attention network task (Posner \& Petersen, 1990), on the relationship between TL-BS variability for food and BMI among Dutch and Chinese children. It was hypothesized that attention control would be negatively related to BMI and would moderate the relationship between TL-BS variability for food and BMI across Dutch and Chinese children: the relationship between TL-BS variability for food and BMI would be stronger in people with less attention control than people with more attention control. Because it was found that BMI was related to executive control and TL-BS variability for emotion-related stimuli was also related to executive control, in Chapter 5, it was expected that weak executive control, measured by stop-signal task, would be associated with both increased attention variability for food and BMI, which might, at least partly, lead to the significant association between TL-BS variability for food and BMI.

The final research goal was to test whether food stimuli are special in the sense that they can still be attention grabbing in a condition of a high perceptual load (Chapter 6). More specifically, perceptual load theory (Lavie, Hirst, De Fockert, \& Viding, 2004) predicts that a high perceptual load of a concurrent task can reduce interference from task-irrelevant distractors, because all perceptual capacity is then used to process task-related information, which leaves no capacity to attend to distractors. Salient cues might be 'special', in the sense that they may still capture attention even when the perceptual load is high. Palatable foods might be such salient stimuli. So, it was hypothesized that palatable food distractors would still attract attention with a high perceptual load, and with a low perceptual load, both food 
and non-food neutral distractors would equally interfere with the concurrent task as compared to the no-distractor condition.

Finally, a discussion and a summary of the main results from all the included studies are presented in Chapter 7, along with directions for future research. 


\section{Chapter 2}

\section{Dynamics of Attentional Bias for Food in Adults, Children, and Restrained Eaters}

Published as:

Liu, Y., Roefs, A., Werthmann, J., \& Nederkoorn, C. (2019). Dynamics of attentional bias for food in adults, children, and restrained eaters. Appetite, 135, 86-92.

https://doi.org/10.1016/j.appet.2019.01.004 


\section{Abstract}

Objective: Empirical evidence of attentional bias (AB) for food in obese and restrained eaters is contradictory. It has been suggested that $\mathrm{AB}$ for food in obese people might reflect both food craving and food-related concerns. Thus, AB for food may be a dynamic process. Methods: A new computational methodology (Zvielli, Bernstein, \& Koster, 2015) was adopted to reanalyze the reaction time (RT) and dwell time of three food-related dot-probe task with eye-tracking studies (Werthmann et al., 2015; Werthmann et al., 2011, 2013). This new computing method uses a sequence of bias scores to express the dynamic changes of AB. Moreover, the variability of RT on filler trials was also calculated. The critical groups in these studies were overweight/obese adults, obese children, and healthy-weight restrained eaters. Results: Both the variability of AB for food stimuli and the variability of RT on filler could significantly predict the variance in body mass index (BMI). When controlling for the variability of RT on filler trials and mean $\mathrm{AB}$ score, larger variability of $\mathrm{AB}$ for food stimuli still existed in obese children and aggregated dataset. The variability of $\mathrm{AB}$ for food stimuli demonstrated no significant correlation with restrained eating scores and dwell time variability. Conclusions: Overweight/obese individuals are characterized by more variability in attention, and this variability is mainly unspecific. It probably reflects less effective executive control ability.

Keywords: attentional bias, dynamics, executive control, obese, restrained eaters 


\section{Introduction}

Currently, people are surrounded by high-caloric palatable foods (Lake and Townshend, 2006) possibly attracting their attention (McSorley, Morriss, \& van Reekum, 2017). According to incentive salience model, such food-related attentional bias (AB) can subsequently result in craving for food, and it might be especially true for overweight and obese people (Roefs, Houben, \& Werthmann, 2015; Frankort et al., 2012; Berridge, Ho, Richard, \& DiFeliceantonio, 2010).

Quite some studies have examined AB for food in individuals of different body weights (e.g., obese, overweight and normal-weight) by using dot-probe task which has most often been used to measure AB. However, empirical evidence for food-related AB in overweight and obese is contradictory (e.g., Roefs et al., 2015; Werthmann, Jansen, \& Roefs, 2015). Briefly, overweight and obese participants showed more, equal or less attention for food cues, compared to lean participants (e.g., Deluchi, Costa, Friedman, Goncalves, \& Bizarro, 2017; Doolan, Breslin, Hanna, \& Gallagher, 2015; Nummenmaa, Hietanen, Calvo, \& Hyona, 2011). The reason for this inconsistency may be partly attributed to the inadequate internal reliability and retest reliability of mean attentional bias scores (Ataya et al., 2012; Schmukle, 2005). Besides, mean AB scores only provide a central tendency of AB but do not provide information at different time points. However, using eye tracking and ERP, two studies found an attentional approach-avoidance pattern in overweight people (Werthmann et al., 2011; Nijs, Franken, \& Muris, 2010). This approach-avoidance pattern might reflect two conflicting goals: eating enjoyment and weight control (Stroebe, van Koningsbruggen, Papies, \& Aarts, 2013), possibly leading dieters to alternate between directing their attention towards and away from food. Given all that, it may be more fitting to consider AB for food as dynamic, reflecting the current motivational state, rather than a stable characteristic (Field et al., 2016, Werthmann, Jansen, \& Roefs, 2016). Mean AB scores may have disguised or canceled out group differences in previous studies.

Zvielli, Bernstein, and Koster (2015) have proposed a new computational methodology to capture the proposed dynamic nature of AB: trial level bias scores (TL-BS). TL-BS consists of a time-series of AB measured over the course of the task. More specifically, in the dot-probe task, each congruent trial is matched with an incongruent trial that is as close 
as possible in time, no further than five trials away (before or after) from the congruent trial. Then, the $\mathrm{AB}$ is calculated by subtracting the response time of each of the congruent trials from its paired incongruent trials, resulting in a time-series of $\mathrm{AB}$ scores. To capture the dynamic nature of AB, TL-BS variability was computed as the mean absolute distance across the whole sequence of TL-BSs, which reflects the extent of fluctuation both toward and away from stimuli over time. Some research found that compared with mean AB scores, TL-BS variability better predicted symptoms in depressed and spider phobic (Zvielli, Vrijsen, Koster, \& Bernstein, 2016; Zvielli et al., 2015). In addition, those with emotion dysregulation exhibited greater TL-BS variability when they were faced with threat-related pictures (Bardeen, Daniel, Hinnant, \& Orcutt, 2017). However, other researchers have critiqued the TL-BS methodology and showed that TL-BS parameters are sensitive to increasing the standard deviation $(S D)$ of reaction time $(\mathrm{RT})$ in the absence of a bias (Kruijt, Field, \& Fox, 2016). According to Kruijt and colleagues (2016), a higher $S D$ may reflect error variance and the TL-BS parameters cannot differentiate measurement error from bias variability. So, TLBS may capture the variability in general responding, and not in threat-related attention. It is important to distinguish whether the variability is specifically for emotion or food-related bias or a more general variability.

Given the potential benefits of the TL-BS, we used it to reanalyze the AB data of three studies (Werthmann et al., 2015; Werthmann et al., 2011, 2013). Because we want to focus on the dynamic nature of attentional bias for food, TL-BS variability was chosen, as it reflects the degree of fluctuation in attention for food across time. The included studies used the dot-probe task to measure RT as well as eye-tracking variables. The studies compared obese and healthy-weight adults (Werthmann et al., 2011), overweight/obese and healthyweight children (Werthmann et al., 2015), and high and low-restrained eaters matched on body mass index (BMI) (Werthmann et al., 2013). Whereas one of these studies did find evidence that overweight/obese adults directed their first gaze more often toward food pictures (Werthmann et al., 2011), no effect was found for the RT-based measure of AB in any of the studies.

The primary purpose was to test whether the variability of $\mathrm{AB}$ (as measured with TL-BS) for food is associated with BMI and restrained eating scores. The second aim was to 
test whether the variability is specific for food stimuli or is observed on filler trials as well. So, the variability of RT on filler trials (mean $S D /$ mean RT) and mean RT on filler trials were selected to express the variability and aggregation in general responding. The third aim was to test whether the variability of $\mathrm{AB}$ for food is related to the variability of eye-tracking variable, dwell time bias, which reflects the maintenance of attention on critical stimuli (Mogg, Field, \& Bradley, 2005). So, the trial-level computing method was used again to calculate the dwell time variability. The final aim was to test whether TL-BS variability can explain more variance than mean $\mathrm{AB}$ scores and the variability of RT on filler trials.

For all the three studies, it was hypothesized that: (1) The variability of AB for food, but not the variability of RT on filler trials would significantly correlate with BMI and restrained eating scores; (2) The variability of $\mathrm{AB}$ for food would be associated with the dwell time variability. (3) The variability of $A B$ for food would explain the variance in BMI and restrained eating scores on top of mean $\mathrm{AB}$ scores and the variability of RT on filler trials.

\section{Analysis 1: TL-BS in overweight/obese and healthy-weight adults}

Analysis 1 reanalyzed reaction time (RT) and gaze dwell time data in the dot-probe task administered to overweight/obese and healthy-weight females (Werthmann et al., 2011). In this study, RT, eye movements, BMI, restrained eating scores, and the consumption of food were recorded. All methods are described in detail in Werthmann et al. (2011), and here a summary of the most relevant information is provided. All the studies received ethical approval.

\section{Method}

\section{Participants}

A total 56 participants female students (age $M=19.45, S D=1.67$; BMI $M=23.90, S D=$ 4.35) took part in this study, including 22 overweight/obese and 34 healthy-weight.

Visual Probe task 
AB was measured with the dot-probe task (MacLeod, Mathews, \& Tata, 1986) while eye movements were recorded. Each trial started with a fixation cross, followed by a pair of pictures, presented side-by-side for $2000 \mathrm{~ms}$. Then, one of the stimuli was replaced by the probe $(*)$, which randomly appeared on the left or right side of the screen, and the probe was presented until the participant pressed the response key. Participants were instructed to focus their gaze first on the fixation cross and then respond to the probe as quickly as possible. This task included incongruent trials (ITs), congruent trials (CTs) and filler trials. On ITs, the probe appeared in the location of the non-food stimulus, whereas on CTs the probe appeared in the location of the food stimuli. Filler trials included two non-food pictures, and the probe appeared in the location of one of them.

The task included 120 trials: 80 critical trials (ITs and CTs) and 40 filler trials. The critical trials consisted of 20 pairs of food and instrument stimuli and filler trials included 10 pairs of nonfood stimuli. All pairs of stimuli were each presented four times.

\section{Taste test}

A bogus taste test was conducted to measure participants' food intake, which was computed by weighing the highly palatable foods before and after the taste test.

\section{Data reduction}

The exclusion criteria of RT were the same as in Werthmann et al. $(2011 ; 2.3 \%$ of data were excluded). Three participants were excluded only from the analyses of consumption as they deviated more than $2 S D s$ from their group mean consumption; three participants were excluded only from the analyses of dwell time as they did not move their eyes on a sufficient proportion $(50 \%)$ of trials (The number of excluded participants is different from Werthmann et al. 2011. They excluded participants with an outlying value on any of the measured dependent variables (i.e., food consumption, eye-tracking variables, RT), whereas we only excluded participants with an outlying value when the variables were used).

\section{The computation of attentional indexes}


Mean AB scores were computed by subtracting the mean RT of congruent from the mean RT of incongruent trials. For the calculation of trial-level bias scores (TL-BS), each congruent trial was matched with an incongruent trial that was as close as possible in time, no further than five trials away. For each pair, the RT or dwell time of the congruent trial was subtracted from the incongruent trial. So, a positive bias score can be interpreted as attention toward food and a negative bias score as attention away from food. TL-BS variability was computed as the sum of absolute distances between sequential TL-BSs on critical trials divided by the total number of TL-BSs (Zvielli et al., 2015). Similar to TL-BS variability, the calculation of dwell time variability was the sum of all absolute distances between sequential trial-by-trial dwell times divided by the total number of dwell times. The variability of RT on filler trials was the mean $S D$ on filler trials divided by the mean RT on filler trials. In our study, the mean number of IT-CT pairs was 56.07 ( $S D=2.05$, range from 52 to 62$)$ and the mean distance between each IT and CT was 1.93 trials $(S D=0.18$, range from 1.5 to 2.32 ) in this study.

\section{Statistical analyses}

The BMI in study 1 had a unimodal distribution, so it was considered as continuous data. First, several zero-order correlations were conducted to test the associations between all attentional variables, BMI, restrained eating scores, and food consumption. Then the hierarchical linear regressions were performed to determine whether TL-BS variability could explain more variance than the variability of RT on filler trials, and mean AB scores for food. It was tested whether the control variables age and food consumption were related to the dependent variable (BMI), in which case they would need to be added to the model. However, age, and food consumption were not related ( $r=.20$ and $.11, p=.13$ and .42$)$. Tests for multicollinearity indicated that a low level of multicollinearity was present (VIF $=1.04$ to 2.17). The independent variables were $\mathrm{z}$-transformed before entering into the statistical model.

\section{Results}

Attentional indexes, BMI, restrained eating, and consumption 
An example of mean AB scores and trial-level bias scores of an overweight and a healthyweight adult was showed in Figure 1. The results of correlations demonstrated that TL-BS variability was significantly related to BMI $(r=.27, p<.05)$, but not to restrained eating and consumption ( $r=.16$ and $.02, p=.25$ and .89 ). The variability of RT on filler trials was significantly related to BMI $(r=.29, p<.05)$, but not to restrained eating or consumption as well ( $r=.17$ and $.02, p=.22$ and .88). The other measures of attention (mean AB scores, mean RT on filler trials, and dwell time variability) were not significantly related to BMI, restrained eating and consumption $(r=-.24$ to $.21, p s=.10$ to .94$)$. TL-BS variability was not significantly related to dwell time variability $(r=.23, p=.10)$, but the two indices of variability, TL-BS variability and the variability of RT on filler trials, were significantly related to each other, $r=.73, p<.01$.

Figure 1. Mean AB scores and trial-level bias scores for one overweight adult and one healthy-weight control adult.

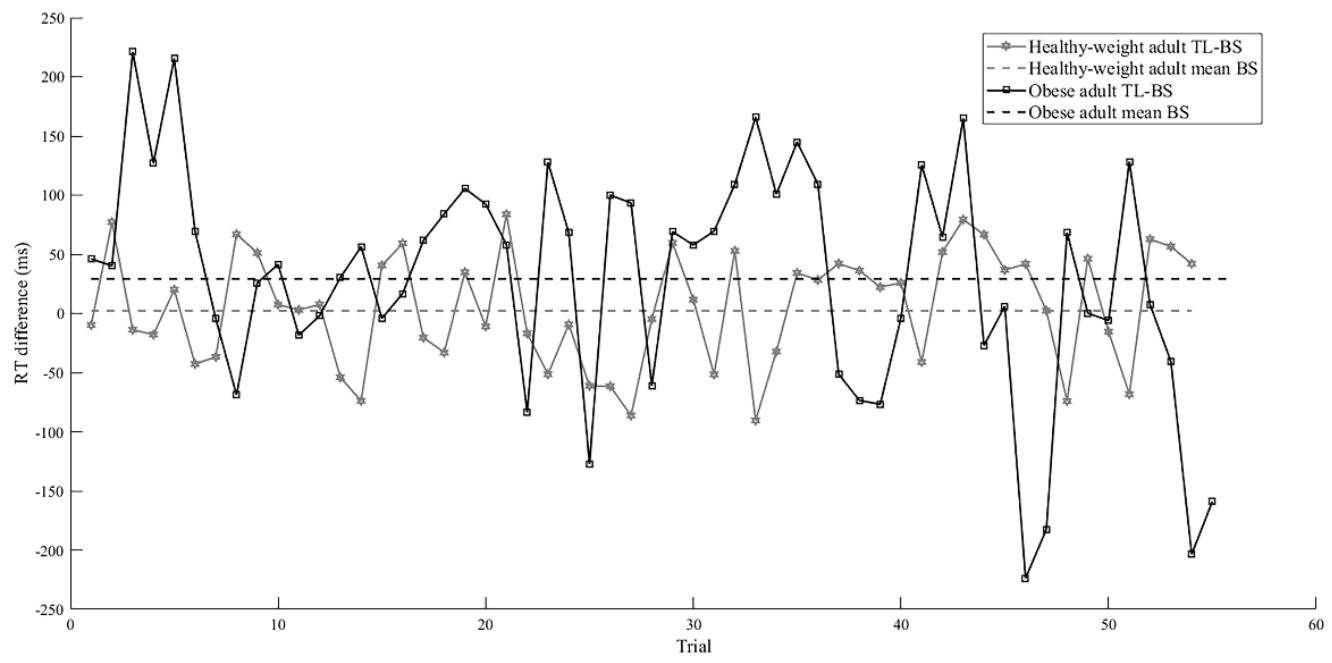

Note. $\mathrm{RT}=$ reaction time; $\mathrm{TL}-\mathrm{BS}=$ trial level bias scores; mean $\mathrm{AB}$ scores $=$ mean attentional bias scores.

\section{Hierarchical regressions}

The results of hierarchical linear regressions demonstrated that the model including only standardized mean $\mathrm{AB}$ scores did not significantly explain variance in BMI. Introducing the Page | 30 
standardized variability of RT on filler trials explained an additional $7.6 \%$ of variation in BMI and this change in $R^{2}$ was significant but adding the standardized TL-BS variability did not significantly improve the model further. See Table 1 for details.

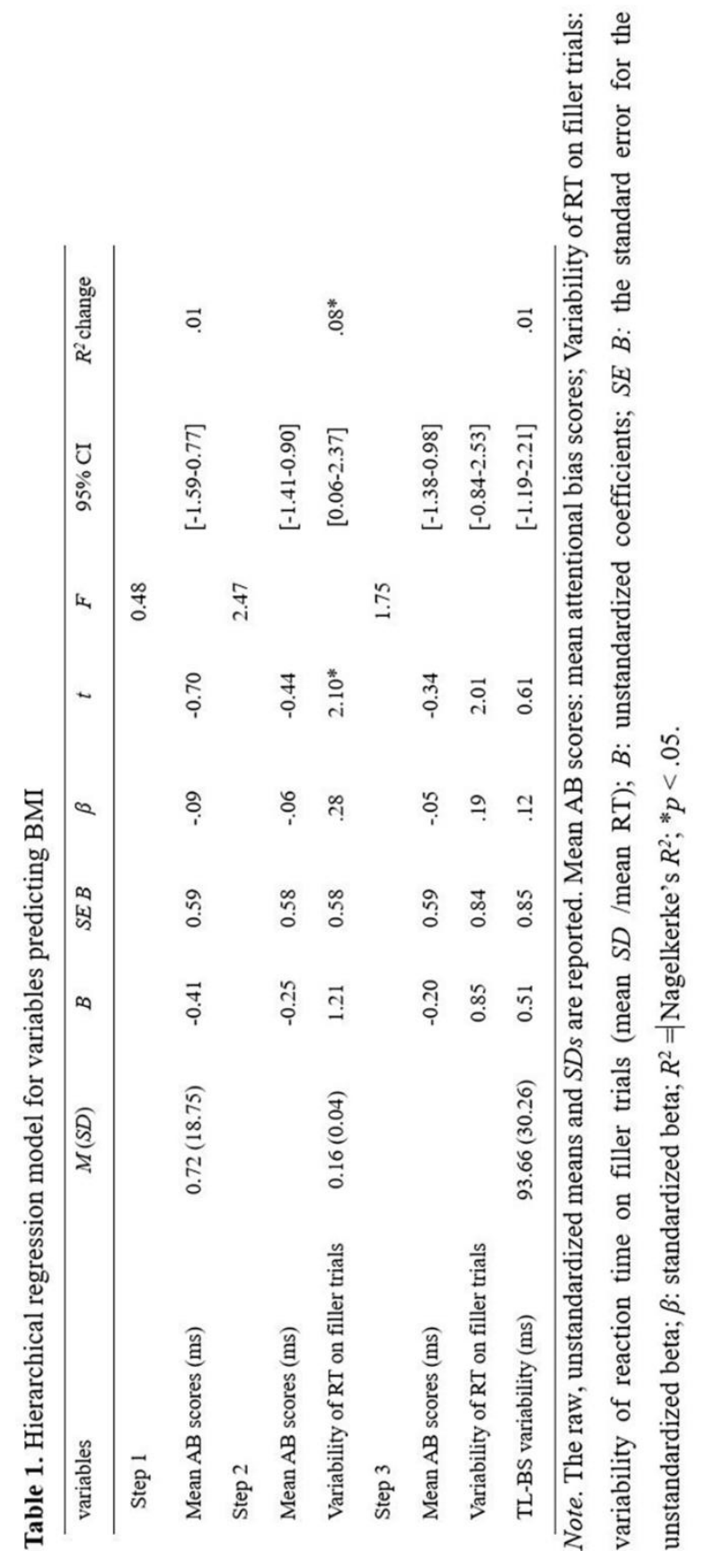




\section{Discussion}

In analysis 1, TL-BS, a measure of variability in attentional bias was used to reanalyze the dot-probe task data in overweight/obese and normal-weight female students (Werthmann et al., 2011). The results showed that TL-BS variability was related to BMI. However, the variability appeared not specific for food stimuli, as the variability of RT on filler trials was also related to BMI. Moreover, TL-BS variability could not explain a significant proportion of variance in $\mathrm{BMI}$ above the mean $\mathrm{AB}$ scores together with variability of $\mathrm{RT}$ on filler trials. So, this suggests that participants with higher BMI, show more fluctuations in their attention to both food and general stimuli. As TL-BS variability showed no significant relation to dwell time variability on food stimuli, it may be concluded that the fluctuations in responding are not specifically related to attention towards or away from food.

\section{Analysis 2: TL-BS in obese and healthy-weight children}

In analysis 2, reaction time (RT) and dwell time data of the dot-probe task administered to obese and healthy-weight children (Werthmann et al., 2015) were reanalyzed. In this study, RT, eye movements and adjusted BMI \% (BMI divided by the national (Dutch) norm BMI (Van Winckel \& Van Mil, 2001), adjusted for sex and age, $\times 100$.) were recorded. A summary of the most relevant information about the method is provided. See Werthmann et al. (2015) for additional details.

\section{Method}

\section{Participants}

34 obese and 36 normal-weight children were recruited. After individual matching on age and sex, 30 obese (age $M=11.91, S D=2.93$; adjusted BMI $\% M=176.05, S D=$ 23.05)/healthy-weight (age $M=11.82, S D=2.99$; adjusted BMI $\% M=97.49, S D=8.95$ ) children pairs were included in the analyses.

Visual probe task 
Each trial started with a central fixation cross (100 ms), and subsequently, the image pair was presented $(3000 \mathrm{~ms})$. Then the probe was presented until the participant responded. The task included two blocks of 40 trials ( 80 trials in total): 64 critical trials and 16 filler trials. The critical trials consisted of 16 pairs of food and animal pictures and filler trials included four pairs. All pairs of pictures were presented 4 times.

\section{Data reduction and the computation of attentional indexes}

The exclusion criteria of RT were the same as in Werthmann et al. (2015; $4 \%$ of data were excluded). The calculation of mean AB scores ${ }^{1}$, TL-BS, TL-BS variability, dwell time variability and the variability of RT on filler trials were the same as analysis 1 . The mean number of matched IT-CT pairs was $44.90(S D=2.34$, range from 39 to 50) and the mean distance between each IT and CT was 1.75 trials ( $S D=0.21$, range from 1.33 to 2.38).

\section{Statistical analyses}

Because of the bimodal distribution of adjusted BMI, independent samples $t$-tests were conducted first to test the effect of weight-status (obese and healthy-weight) on mean $\mathrm{AB}$ scores, TL-BS variability, dwell time variability, mean RT on filler trials, and variability of RT on filler trials separately. Then we used binary logistic hierarchical regressions to detect whether TL-BS variability could explain additional variance above and beyond mean $\mathrm{AB}$ scores and variability of RT on filler trials. All predictors were z-standardized and they demonstrated a low level of multicollinearity, VIF $=1.02$ to 1.59 . Rank-order correlations were computed to detect whether age and sex were related to adjusted BMI. As the correlations were not significant, age and sex were not included in the regression model $(r=$ -.11 and $-.03, p=.39$ and .80$)$.

\section{Results}

\footnotetext{
${ }^{1}$ The computation method of the mean BS in analyses 2 and 3 is slightly different from Werthmann et al. (2013) and Werthmann et al. (2015), in which they divided response time into five different conditions (congruent dot left, congruent dot right, incongruent dot left, incongruent dot right and filler) and then calculated the BS.
} 


\section{Attentional indexes and group status}

The results of $t$-tests showed that mean AB scores did not significant differ between obese and healthy weight children, $t(1,58)=1.60, p=.12$. Obese children did show larger TL-BS variability than healthy-weight children, $t(1,58)=3.77, p<.01$. The variability of RT on filler trials was marginally significantly larger in the obese children than in the healthyweight children, $t(1,58)=1.89, p=.06$. There was no significant difference between the weight groups in dwell time variability $t(1,58)=0.84, p=.40$, and in mean RT on the filler trials, $t(1,57)=0.67, p=.51$. TL-BS variability was significantly related to the variability of RT on filler trials $(r=.60, p<.01)$ but not to dwell time variability $(r=-.06, p=.64)$.

\section{Hierarchical regressions}

The results demonstrated that adding the standardized mean AB scores in the first step did not significantly predict weight status. The variability of RT on filler trials in the second step significantly increased the explained variance of weight-status. Finally, the standardized TLBS variability was added in the third step, and it significantly increased the proportion of explained variance further. See Table 2. 


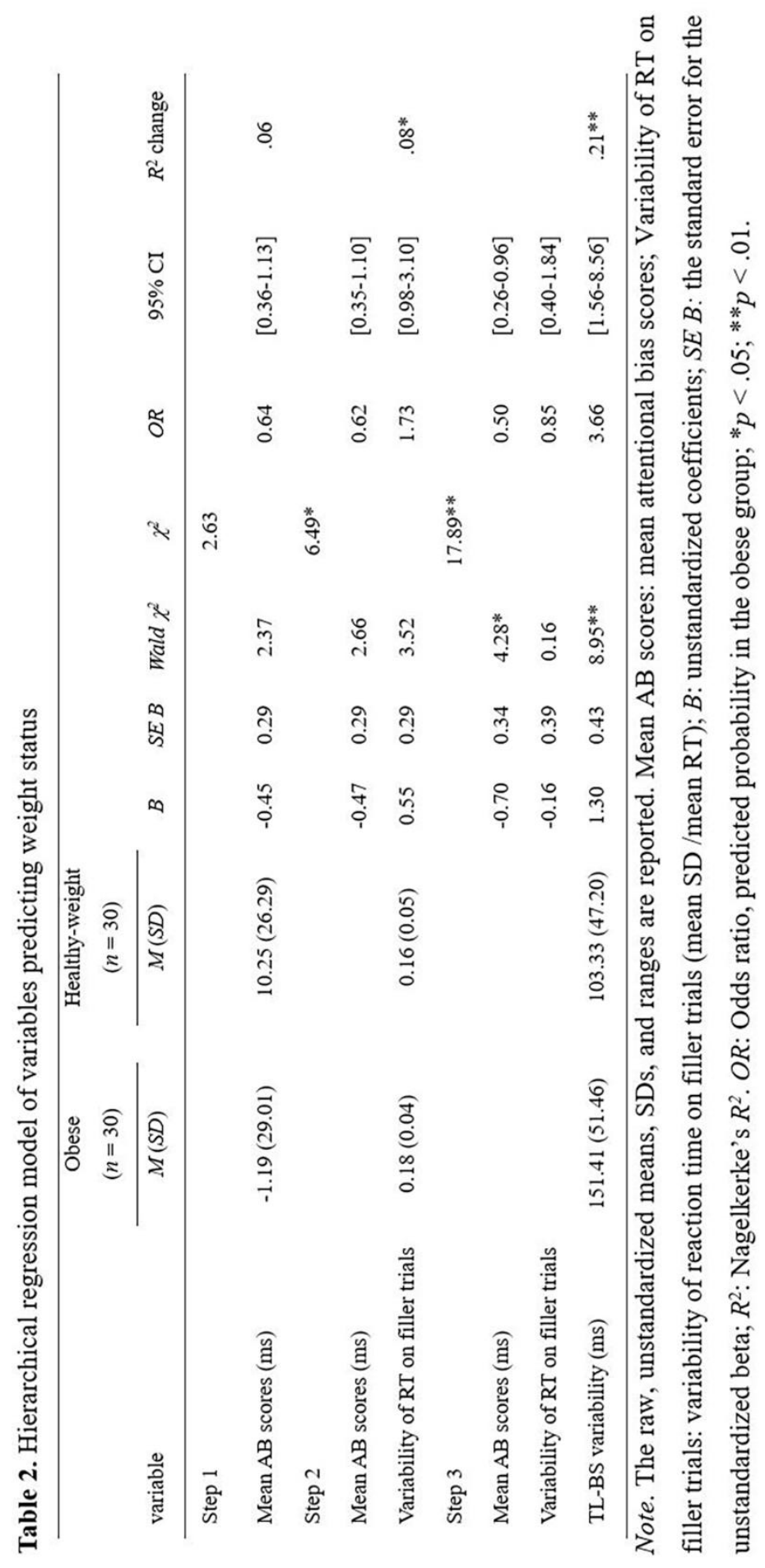




\section{Discussion}

In analysis 2, we used TL-BS to reanalyze the dot-probe task data in obese and normal-weight children. In line with our hypotheses, the results indicated that obese children demonstrated more variability of attentional bias for food (TL-BS variability) than healthy-weight children and the variability of attentional bias for food significantly predicted weight status when controlling for the variability of RT on filler trials and mean AB scores. Inconsistent with our hypothesis, the variability of RT on filler trials also explained marginally more variance above mean AB scores. In addition, dwell time variability showed no significant association with TL-BS variability and was not different between the two weight groups. Possibly, TLBS based on reaction time may capture different aspects of attention than those reflected in dwell time. The results of analysis 2 are partly in line with the results of analysis 1 , in that both weight groups did not differ on dwell time variability, and both overweight/obese adults and obese children showed increased variability on $\mathrm{AB}$ for food and on general reaction time. However, for obese children, specifically the variability of attentional bias for food was a better predictor of weight status than the variability of general responding.

\section{Analysis 3: TL-BS in restrained and unrestrained eaters}

Analysis 3 reanalyzed reaction time (RT) and dwell time data of dot-probe task administered to healthy-weight restrained and healthy-weight unrestrained eaters (Werthmann et al., 2013). In this study, RT, eye movements, restrained eating scores, and BMI were recorded. All methods are described in detail in Werthmann et al. (2013), and a summary of the most relevant information is provided.

\section{Method}

\section{Participants}

45 female participants were recruited (age $M=21.67, S D=2.05$; BMI $M=21.46, S D=1.61$ ) and their restrained eating scores $(M=11.76, S D=5.50)$ were rated by Restraint Scale (Herman \& Polivy, 1980). The groups were matched on BMI. 
Visual probe task

The description of the dot-probe task was the same as for analysis 1 .

Data reduction and the computation of attentional indexes

The exclusion criteria of RT were the same as in Werthmann et al. (2013; $2.3 \%$ of data were excluded.) The calculations of all the attentional indexes were the same as analysis 1 . The mean number of matched IT-CT pairs was 56.11 ( $S D=1.98$, range from 52 to 60) and the mean distance between each IT and CT was 1.92 trials $(S D=0.18$ range from 1.59 to 2.44) in this study.

\section{Statistical Analyses}

The distribution of restrained eating scores was unimodal, so we first conducted zero-order correlations and then proceded with hierarchical linear regressions, with z-standardized variables, in which we added the mean $\mathrm{AB}$ scores in the first step, the variability of RT on filler trials in the second step, and the TL-BS variability in the final step. A low level of multicollinearity was present in this study $(\mathrm{VIF}=1.00$ to 4.30$)$. (VIF $=1.00$ to 4.30$)$. The control variables age and hunger were not related to the restrained eating scores and were therefore not included in the hierarchical regressions ( $r=-.04$ and $.12, p=.80$ and .43 ).

\section{Results}

\section{Attentional indexes and restrained eating}

The restrained eating scores were not correlated significantly with TL-BS variability, mean $\mathrm{AB}$ scores, dwell time variability, mean RT on filler trials, and the variability of RT on filler trials ( $r=-.23$ to $.21, p s=.14$ to .56 ). In addition, dwell time variability did not correlate significantly with TL-BS variability $(r=.12, p=.43)$. However, TL-BS variability was related significantly to the variability of RT on filler trials $(r=.88, p<.01)$.

\section{Hierarchical regressions}

Results showed that the mean $A B$ scores did not significantly explain variance in restrained eating. Adding the variability of RT on filler trials and TL-BS variability did not significantly 
increase the proportion of explained variance in restrained eating scores. See Table 3 for details.

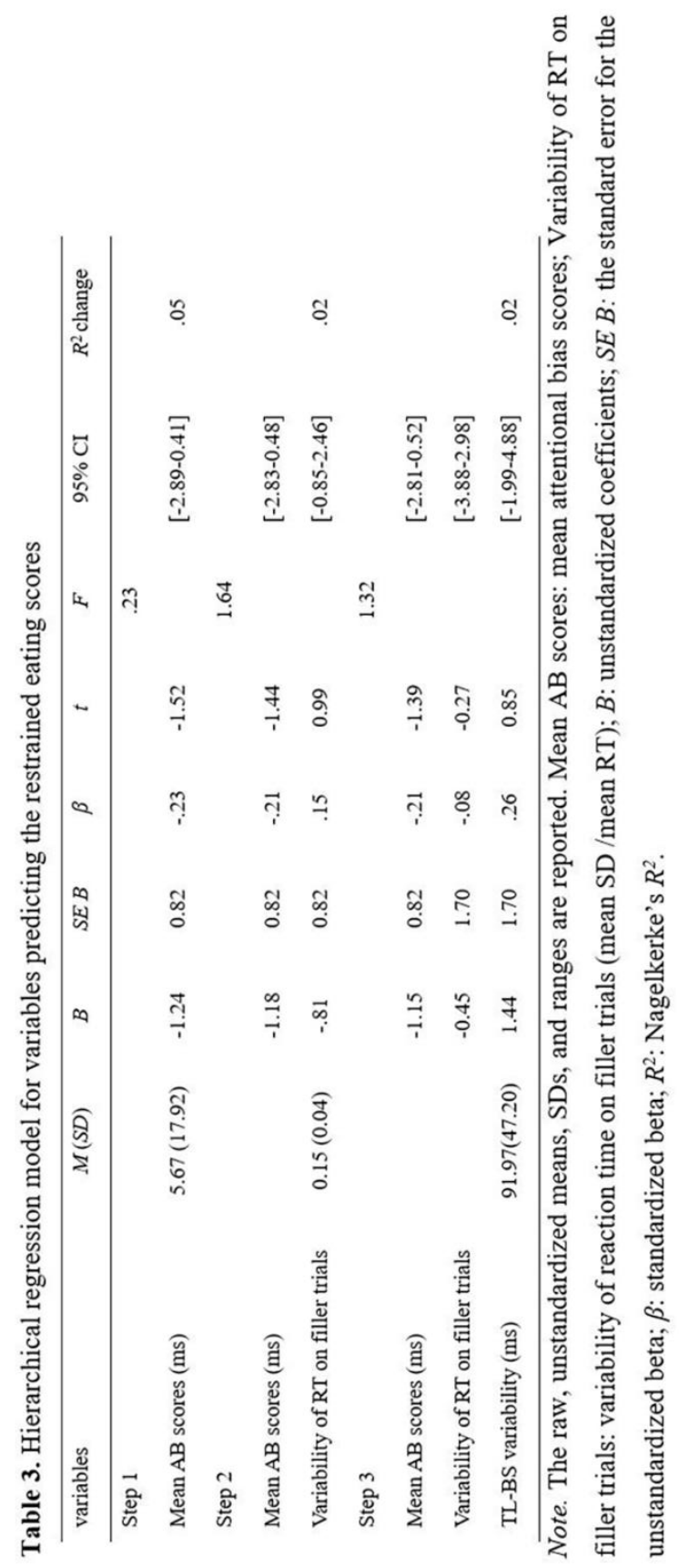

Page | 38 


\section{Discussion}

In analysis 3, we used the TL-BS computational method to reanalyze the data in normalweight restrained and unrestrained eaters. Different from our prediction, the results indicated that (1) TL-BS variability was not related significantly to restrained eating scores and could not explain additional variance in restrained eating as well. (2) Dwell time variability was not correlated significantly with TL-BS variability. The results suggest that there is no significantly relation between restrained eating and food-related attentional dynamics. This possibly means restrained eaters are indeed not characterized by dynamic changes in foodrelated $\mathrm{AB}$ or TL-BS is not an adequate way to measure this. Comparing with the results of study 1 and study 2, it seems that BMI instead of restrained eating scores is more likely associated with the variability of $\mathrm{AB}$ for food or general responding. Again, the TL-BS variability did not show any association with dwell time variability, so it is necessary to reconsider what TL-BS reflects.

\section{Meta-analysis}

As all the three studies only have modest sample sizes, lack of sufficient power might be a problem. To increase the power, we aggregated the data of three studies into one dataset. Our purposes were in line with the above studies. However, we do not have restrained eating scores in study 2 and from the results of our three studies, it seems TL-BS variability is more likely related to BMI instead of restrained eating scores, so we did not present the results including restrained eating scores here (the relevant information can be found in the supplement).

As we calculated the percentage adjusted BMI of children according to Dutch norm BMI, to create one dataset, we calculated the adjusted BMI \% for the adults in study 1 and study 3 as well as study 2 . Two outliers ( $3 S D$ above the mean) in the adjusted BMI \% were replaced by the nearest adjusted BMI $\%$ of the whole sample (see Wilcox, 2001) ${ }^{2}$. After aggregated the datasets, 161 participants (158 for dwell time variability) were included in the

\footnotetext{
2 The analyses with unchanged values or exclusion of these values showed the same patterns and would lead to the same conclusions.
} 
analysis (age $M=17.24, S D=4.83$; adjusted BMI $M=116.61, S D=32.21$ ), including 30 males and 131 females.

Because of the unimodal distribution of the adjusted BMI \%, we first conducted the correlations and then used hierarchical linear regressions with z-standardized variables. As age was related to the adjusted BMI \% $(r=-.43, p<.01)$, age was controlled in both correlations and regressions. Tests for multicollinearity indicated a low level of multicollinearity (VIF $=1.01$ to 2.38$)$.

The results of partial correlations demonstrated that TL-BS variability $(\mathrm{r}=.31, p$ $<.01)$, mean $\mathrm{AB}$ scores $(\mathrm{r}=-.18, p<.05)$, dwell time variability $(\mathrm{r}=.19, p<.05)$, and the variability of $\mathrm{RT}$ on filler trials $(\mathrm{r}=.21, p<.01)$ were all significantly correlated with adjusted BMI \%. In addition, TL-BS variability was positively related to the variability of RT on filler trials $(r=.68, p<.01)$, but not related to dwell time variability $(r=-.01, p=.95)$. For the hierarchical linear regressions, the model including only standardized mean AB scores significantly explained variance in BMI after controlling for age. Introducing the variability of RT on filler trials significantly improved the model. Finally, adding the standardized TLBS variability in the last step explained an additional $4.1 \%$ of the variation in BMI and this change in $R^{2}$ was significant. See table 4.

According to the results of mega-analysis, we can conclude that there was a significant associated between TL-BS variability and adjusted BMI \%, and such associations also exist in the variability of RT on filler trials. However, TL-BS variability for food was the best predictor of adjusted BMI \% and could significantly explain variance in \% BMI after controlling for age, mean AB and variability of RT on filler trials. Again, TL-BS was not related to variability in dwell time, which suggests that TL-BS might not reflect attention towards and away from food. 


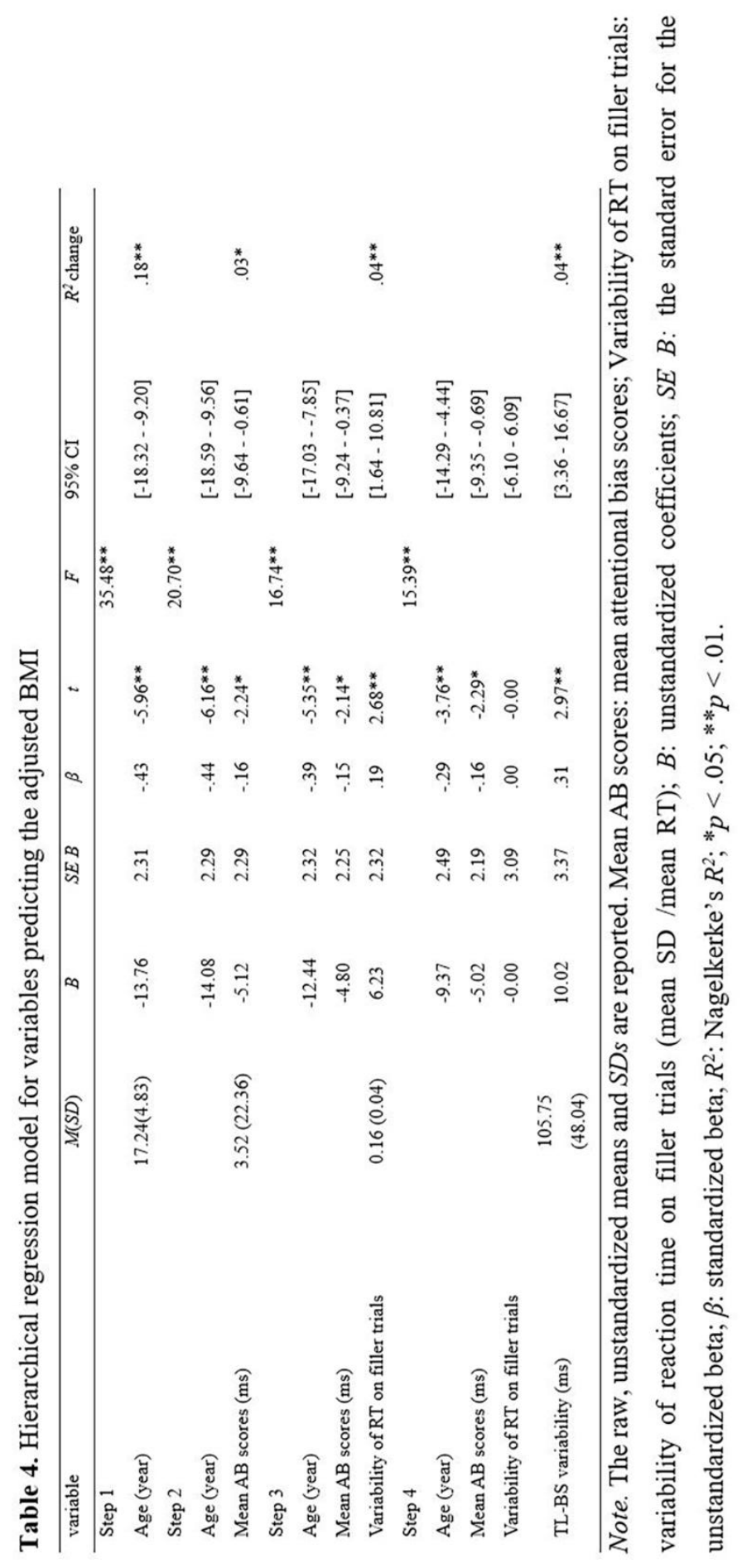




\section{General discussion}

Previous research on food-related AB did not produce consistent results (e.g., Roefs et al., 2015; Werthmann et al., 2015). Recently, several studies used a new computational method, TL-BS, to calculate variability of the attentional bias (AB) data in emotion-related dot-probe tasks and produced more consistent results (Zvielli et al., 2016; Zvielli et al., 2015). However, this TL-BS approach has not been taken yet in research on biased attention for food.

In the current paper, we used this novel computational method to reanalyze $\mathrm{AB}$ data in three different groups, overweight/obese adults, obese children, and normal-weight restrained eaters, all compared to control groups. We also chose the variability of RT on filler trials (mean SD /mean RT) to measure the variability on general responding. The main findings were (1) Overall, people with higher BMI demonstrated larger TL-BS variability for food (study 1, 2 and meta-analysis). (2) When controlling for the variability of RT on filler trials and mean AB scores, TL-BS variability could still significantly predict the variance in BMI (study 2 and meta-analysis, although not in study 1). (3) Both overweight/obese adults and obese children demonstrated larger variability of RT on filler trials (study 1, 2 and metaanalysis). (4) The variability of attentional bias for food was not correlated significantly with dwell time variability and restrained eating scores (all studies).

From all the studies, we can conclude that overweight/obese women and obese children all are characterized by higher variability of attentional bias for food and general responding. The variability of food-related attentional bias and general responding may due to the executive control ability, as overweight/obese people showed impairments of executive function (Yang, Shields, Guo, \& Liu, 2018). The relation between restrained eating and executive functioning is less clear, with inconsistent results in the literature (Meule, Lukito, Vögele, \& Kübler, 2011; Nederkoorn et al., 2004) and together with the present results this might suggest that restrained eating is not related to diminished executive functioning. However, some research suggested that executive functioning might be specifically related to uncontrolled eating or unsuccessful dietary restrained and not to restrained eating in general (Martin, Davidson \& McCrory, 2018; Van Koningsbrugge, Stroebe \& Aarts, 2013). In future research, it should be tested if unsuccessful dieting, instead of general restrained, is related to increased variability in food-related attentional bias or to general responding. 
The results of study 2 and mega-analysis showed that the variability of AB for food could predict additional variance of BMI above the variability of general responding. It demonstrates the necessity to consider the role of time when measuring attentional bias for food and merely focus on the aggregated mean scores cannot draw the whole picture of attentional bias for food. It also provides us with the evidence that people with higher BMI may have more approach-avoidance towards food. However, the reanalyzes all revealed that dwell time variability was not correlated with TL-BS variability. Dwell time is defined as the maintenance of attention on critical stimuli (Mogg et al., 2005). The bigger dwell time represents participants lingering longer on critical stimuli (towards target) and vice versa. It seems therefore reasonable to consider that TL-BS variability may not link to attention towards or away from food. Hence, it is difficult to indicate what the variability of attentional bias for food (TL-BS variability) reflects in our current paper. More research is needed in which TL-BS is validated, for instance by relating it to more other measures of goal conflict. The underlying mechanism on the variability of attentional bias is unclear, but these results do show TL-BS can predict the variance in BMI, so this new direction is interesting.

Several limitations should be mentioned. First, TL-BS variability can only capture the degree of fluctuation, but it cannot provide us with the frequency of bias switches, which is also a critical index to weigh the dynamic changes of AB. It still needs great efforts to develop a better method to capture the dynamic nature of AB. Second, our participants in study 1 and study 3 only included female participants, so the results cannot be generalized to males.

In summary, the present findings show that the variability of attention in both obese/overweight women and children is not specific for food stimuli but is also apparent for non-food stimuli, whereas the larger variability of $\mathrm{AB}$ for food seems to characterize obese people, especially in obese children, better than the variability of general responding. The variability of $\mathrm{AB}$ for food showed no significant correlation with dwell time variability and restrained eating scores. These findings suggested that TL-BS can partly predict the variance in BMI, but it is not clear what it actually reflects, casting doubt on the validity of the measure. More research is needed to test the relation between general attention, attention for food, food intake, and overweight/obesity. 


\section{supplementary materials}

\section{Mega-analysis for restrained eating}

The data of study 1 and study 3 was aggregated to increase the power of analysis related to restrained eating. Finally, 101 female participants were included in the analysis (age $M=$ 20.44, $S D=2.15$; restrained eating scores $M=11.56, S D=5.31)$. As the unimodal distribution of restrained eating scores, it was considered as continuous data. The potential confounder age was not related to the restrained eating scores $(r=.13, p=.20)$, therefore there was no need to control it. The results of correlations showed that restrained eating scores were not significantly related to mean AB scores, TL-BS variability, dwell time variability, mean RT on filler trials, and the variability of RT on filler trials $(r=-.06$ to $.19, p s=.06$ to .68). Then the hierarchical linear regressions were conducted using $\mathrm{z}$-standardized variables and test for multicollinearity indicated a low level of multicollinearity (VIF = 1.02 to 3.79). Results demonstrated that the mean $\mathrm{AB}$ scores did not significantly explain the variance in restrained eating. Adding variability of RT on filler trials and TL-BS variability did not significantly increase the proportion of explained variance in restrained eating. See table $1 \mathrm{~s}$. 


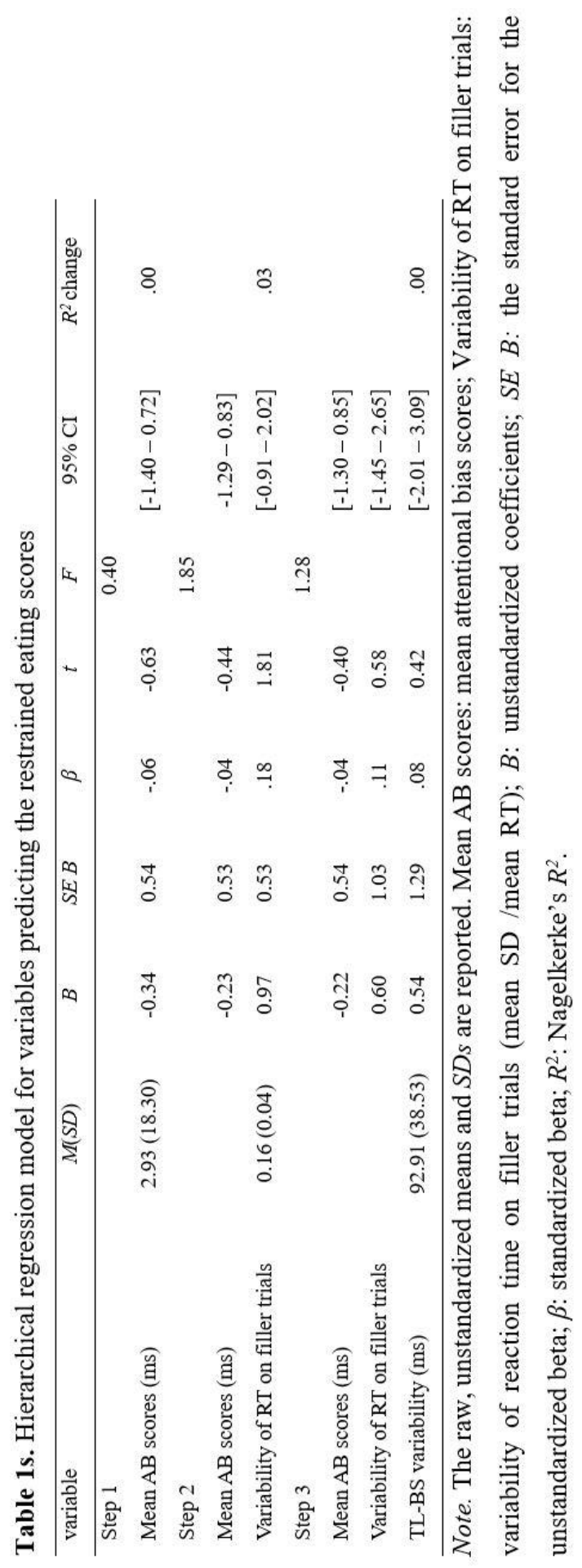




\section{Chapter 3}

\section{Food Palatability Directs Our Eyes Across Contexts}

Published as:

Liu, Y., Roefs, A., \& Nederkoorn, C. (2021). Food Palatability Directs Our Eyes Across Contexts. Frontiers in Psychology, 12, 1655. https://doi.org/10.3389/fpsyg.2021.664893 


\begin{abstract}
It is often believed that attentional bias (AB) for food is a stable trait of certain groups, like restrained eaters. However, empirical evidence from this domain is inconsistent. High-calorie foods are double-faceted, as they are both a source of reward and of weight/health concern. Their meaning might depend on the food-related context (i.e., focus on health or on enjoyment), which in turn could affect $\mathrm{AB}$ for food. This study primed 85 females with a hedonic, healthy, and neutral context successively, and examined whether food-related context affected $\mathrm{AB}$ for food and if effects were moderated by dietary restraint. Both the mean tendencies of $\mathrm{AB}$ for food and variability of $\mathrm{AB}$ for food were assessed in a food dotprobe task with a recording of both reaction times and eye movements. Contrary to our hypotheses, $\mathrm{AB}$ for food was not significantly affected by either context or the interaction between context and dietary restraint. Instead, liking of the presented food stimuli was related to longer initial fixations and longer dwell time on the food stimuli. In addition, in line with prior research, $\mathrm{BMI}$ was correlated with variability of $\mathrm{AB}$ for food instead of mean $\mathrm{AB}$ for food. In conclusion, this study did not find any support that $\mathrm{AB}$ for food is dependent on food-related context, but interestingly, reaction time-based variability of $\mathrm{AB}$ for food seems to relate to $\mathrm{BMI}$ and eye movement-based mean $\mathrm{AB}$ seems to relate to appetitive motivation.
\end{abstract}

Keywords: attentional bias; dynamics; context; priming; restrained eating 


\section{Introduction}

In general, people are naturally attracted by high-calorie foods (e.g., McSorley, Morriss, \& van Reekum, 2017). In the Western food-rich environment, the abundant presence of high caloric palatable foods represents salient cues that can induce food craving (Hill \& Peters, 1998), subsequent food intake, and ultimately weight gain (Boswell \& Kober, 2016). However, in daily life, there are also moments that weight control thoughts or weight/healthrelated cues can lead to food avoidance. High-calorie foods are frequently craved but are also often a source of worry and weight concern. This is also referred to as the double-facetted nature of food (Roefs, Fransen, \& Jansen, 2018), in other words, a conflict between food enjoyment and weight concern. The current study investigates if inducing a hedonic versus a health context affects attentional bias $(\mathrm{AB})$ for food and if this effect is moderated by dietary restraint.

$\mathrm{AB}$ refers to an enhanced attention to salient or relevant stimuli (Drobes, Oliver, Correa, \& Evans, 2019). In previous studies, AB for food was mostly considered as a traitlike characteristic of both restrained eaters (REs) and people with overweight/obese. This popular belief is based on the incentive salience model: a reward stimulus, like palatable food, can lead to a craving for it, which reflects on a biased attention to the rewarding food and such attention-grabbing should be especially true for REs and people with overweight/obese (e.g., Berridge, 2009; Nijs \& Franken, 2012). However, the empirical evidence for foodrelated $\mathrm{AB}$ in REs and people with overweight/obesity is inconsistent, which has been repeatedly revealed by reviews and meta-analyses (e.g., Doolan, Breslin, Hanna, \& Gallagher, 2015; Hagan, Alasmar, Exum, Chinn, \& Forbush, 2020; Hardman et al., 2020; Roefs, Houben, \& Werthmann, 2015; Werthmann, Jansen, \& Roefs, 2015). Briefly, some studies indeed found that people high in BMI or dietary restraint biased their attention more to food stimuli than the control group (e.g., Hume, Howells, Rauch, Kroff, \& Lambert, 2015; Kemps, Tiggemann, \& Hollitt, 2014; Meule, Vögele, \& Kübler, 2012), while other studies found that people high in BMI or dietary restraint showed equal (e.g., Doolan, Breslin, Hanna, Murphy, \& Gallagher. 2014; Hodge, Han, \& McBride, 2020; Werthmann et al., 2013) or even less (e.g., Fang et al., 2019; Nathan et al., 2012) attention to food cues as compared to their counterparts. Interestingly, some studies even revealed an attention approach-avoidance pattern in people high in BMI and dietary restraint (e.g., Hollitt, Kemps, Tiggemann, Smeets, 
\& Mills, 2010; Werthmann et al., 2011). This inconsistency might partly be explained by the fact that diverse measurements were used to capture individuals' AB for food (e.g., different paradigms and materials used to assess $\mathrm{AB}$ for food). It could also be due to some studies being underpowered, which possibly could have led to biased results. However, we believe that $\mathrm{AB}$ for food is not a stable trait-like feature in people with overweight/obesity and REs.

Apart from the inconsistent empirical evidence, linking enhanced $\mathrm{AB}$ for food to people high in BMI or dietary restraint also conveys two incorrect beliefs: (1) food is only a source of reward; (2) attention is stable over time, either towards or away from food. As we stated before, palatable foods can both a source of reward and weight gain/health concern (Roefs et al., 2018). Relevant for the second incorrect belief is that attention is also a reflection of the current motivation (top-down) (Connor, Egeth, \& Yantis, 2004). Therefore, theoretically, whether an individual focuses on the enjoyment facet or the health facet of food could direct attention towards or away from food, which might have contributed to the inconsistent results across studies. Previous studies indeed provided evidence that it is possible to influence individuals' AB for high-calorie foods and subsequent food intake by manipulating the context. For example, in an online supermarket eye-tracking study, priming health/diet cues (recipe banners containing health and dieting words were presented during food choice) increased low-calorie food choices, decreased high-calorie food choices, and increased total dwell times on low-calorie products (van der Laan, Papies, Hooge, \& Smeets, 2017). Papies and Hamstra (2010) found that priming dieting cues (a weekly recipe that was "good for a slim figure" and low in calories was attached to the door of the butcher's store) decreased food consumption in REs, but not in unrestrained in unrestrained eaters (uREs). In line with this, a dieting context (participants were asked to choose a one-day menu from a healthy menu card to their best friend who wants to lose weight) decreased mean AB scores for high-caloric food only in participants with higher dietary restraint scores (Werthmann, Jansen, \& Roefs, 2016). All in all, evidence supports the idea that individuals' AB for food is not stable and is influenced by food-related contexts, especially in high REs.

In the tasks used to measure $\mathrm{AB}$, an aggregated mean $\mathrm{AB}$ score has been widely adopted to characterize $\mathrm{AB}$ for food, which reflects an overall, stable tendency of $\mathrm{AB}$ during the task. However, this mean AB score does not do justice to the potentially dynamic nature of $\mathrm{AB}$ for food, as attention to food might alternate between approach and avoidance, even 
within one study within one participant. Interestingly, another method of computing AB was introduced: trial-level-bias scores (TL-BS; Zvielli, Bernstein, \& Koster, 2015), which specifically acknowledges that $\mathrm{AB}$ might not be a fixed characteristic, but may instead fluctuate over the course of an experiment. This method focuses on the degree of fluctuation in $\mathrm{AB}$, and how this fluctuation is related to certain traits. TL-BS has shown added value in several domains. That is, studies revealed that TL-BS variability, one of the parameters to measure the variability of AB for critical stimuli based on the TL-BS, could better predict BMI, depression, and spider phobia than corresponding mean AB scores (Liu, Nederkoorn, Roefs, 2019; Liu, Roefs, Werthmann, Nederkoorn, 2019; Zvielli et al., 2015; Zvielli, Vrijsen, Koster, \& Bernstein, 2016). Specifically, for AB for food, it has been shown that people with a higher BMI do not have significantly more $\mathrm{AB}$ for food than healthy-weight people, but are characterized by more TL-BS variability for food (Liu, Nederkoorn et al., 2019; Liu, Roefs et al., 2019).

Altogether, both empirical and theoretical evidence support that individuals' AB for food is not a stable trait but fluctuates over time. Attention fluctuations might reflect the momentary inner conflict between food enjoyment and weight/health concern when confronted with palatable food, which possibly can be shaped by manipulating food-related contexts. Moreover, individuals with higher dietary restraint are more sensitive to both foodrelated reward and punishment (Ahern, Field, Yokum, Bohon, \& Stice, 2010), and as we mentioned before, it was found that the influence of context on food intake and $\mathrm{AB}$ for food only happened in participants high in dietary restraint (Papies \& Hamstra, 2010; Werthmann et al., 2016). Therefore, it might be that high REs who frequently experience conflicts between food enjoyment and weight concern (Stroebe, van Koningsbruggen, Papies, \& Aarts, 2013), have more fluctuations in AB for food when in a 'neutral state', reflecting what might typically occur in daily life, fluctuating between craving and weight concern when confronted with palatable food. These fluctuations might reduce when the context either strongly emphasizes enjoyment or health. Specifically, people may have more and more consistent $\mathrm{AB}$ towards high-caloric food when in a hedonic context, and more and more consistent $\mathrm{AB}$ away from high caloric food when in a health context. Relevant to mention here is that mindset or context was not manipulated in previous studies that used TL-BS AB 
scores. It would therefore be valuable to see how the food-related contexts influence the variability of $\mathrm{AB}$ for food.

The current study examined the effect of context priming on both the average $\mathrm{AB}$ for food and the variability of $\mathrm{AB}$ for food. Context (hedonic, health, and neutral) was manipulated in a within-subject design. It was hypothesized that (1) in the hedonic context as compared to the health context, participants would show a larger mean $\mathrm{AB}$ towards food, and have longer first fixations and dwell time on food stimuli, whereas the neutral context was expected to fall in between the hedonic and health context; (2) compared with the neutral context, participants would show fewer RT-based and EM-based fluctuations on food in both the hedonic and the health context; (3) contexts would affect both the average and the fluctuations of $\mathrm{AB}$ for food more for participants scoring higher on dietary restraint.

\section{Materials and Methods}

The current study was approved by the Ethical Committee of the Faculty of Psychology and Neuroscience, Maastricht University, and all participants signed the informed consent, in which they were informed about the procedure, storage of data and their right to withdraw from participation without any consequence. This study was pre-registered at AsPredicted (https://aspredicted.org/96j53.pdf).

\section{Participants}

91 female participants were recruited via posters on the university campus (Maastricht University, the Netherlands) or on the Facebook, or via an online recruitment system. To disguise the true purpose of this study and avoid influencing participants' behavior, it was informed on the poster that this study aims to investigate "attention patterns for different objects". People who signed up for the study received a screening questionnaire with questions on sex, age, and vision. Females with a normal or correct to normal vision and above 16 years old were invited for participation. Because food pictures depicting meat were included in the study, after the participant finished the experiment, she was asked whether she adhere to a vegetarian or vegan diet. Six participants were excluded from analyses because of either a vegetarian or vegan diet. So, 85 participants were included in the analyses. Participants' characteristics can be found in Table 1 . The sample size is adequate to detect a 
medium effect size for the main aim of the study, which is to test the effect of priming condition (within-subject) on all measures of $\mathrm{AB}$ scores, thus repeated measures of MANOVAs were conducted. When using Repeated measures of MANOVAs (number of groups $=1$, number of measurements $=3$, medium effect size $\mathrm{f}=0.25$, alpha $=0.05$, power $=0.95)$, the estimated required sample size was 45 .

Table 1. Participant Characteristics.

\begin{tabular}{lccc}
\hline variables & $M$ & $S D$ & Range \\
\hline RS score & 13.80 & 5.20 & $1.00-28.00$ \\
Age & 21.48 & 2.96 & $17.00-31.00$ \\
BMI & 22.02 & 3.01 & $16.81-31.25$ \\
Hunger & 33.40 & 23.74 & $0.00-80.00$ \\
Food liking & 57.12 & 19.91 & $6.50-92.50$
\end{tabular}

Note. RS: restraint scale (Herman \& Polivy, 1980); BMI: body mass index; hunger: hunger level of participants (0-100 VAS); food liking: average food liking score for the high-calorie food stimuli presented in the food-dot probe task (0-100 VAS)

\section{Measurements}

\section{Dietary Restraint}

The Restraint Scale (RS, Herman \& Polivy, 1980) was used to measure restrained eating. The $\mathrm{RS}$ is an 11-item self-report scale that is used to assess chronic dieting. In this study, Cronbach's alpha was 0.76 .

\section{Hunger Level}

Participants' hunger level was measured on an online 100-mm visual analogue scale (VAS) by asking "How hungry are you right now?", with 0 indicating absolute lack of hunger and 100 indicating extreme hunger.

\section{Liking of the food images}


Liking of the high-calorie food images that were presented in the food-dot probe task (20 images) was measured on an online 100-ms VAS, with 0 indicating a lack of liking and 100 indicating extreme liking.

\section{Food Dot-probe Task}

AB for high caloric foods was measured using the food dot-probe task with a recording of both reaction times and eye movements. The task was presented using Experiment Builder (SR Research, Ontario, Canada).

Trial procedure. Priming context was manipulated in a blocked fashion, and in each block, one type of priming picture (hedonic or health or neutral) was presented in each trial. Each trial began with a central fixation dot, which disappeared directly after participants fixated on it. Subsequently, a prime image was presented for $1000 \mathrm{~ms}$. After presentation of another central fixation dot $(500 \mathrm{~ms})$, two images were simultaneously presented side-by-side for $2000 \mathrm{~ms}$. Next, one of the images was replaced by the probe $(*)$, which randomly and equally often appeared on the left or right side of the screen. The probe was presented until the participant's response or for a maximum of $2000 \mathrm{~ms}$. Participants were instructed to focus on the central fixation and respond to the probe as quickly as possible.

Trial types. Three different types of trials were included: food-incongruent trials (ITs), food-congruent trials (CTs), and neutral-neutral trials (filler trials). On the ITs and CTs, a high calorie-food and a musical instrument picture were presented, whereas on the filler trials, two neutral nonfood pictures (e.g., office supplies) were presented. During ITs, the probe appeared in the location of the musical instrument picture, whereas during CTs, the probe appeared in the location of the food picture. In the filler trials, the probe appeared randomly and equally often on the left and right side.

Block types. The task included one practice block, two buffer blocks, and three priming blocks. The 3 different trial types were evenly and randomly distributed across the priming blocks (40 CTs, 40 ITs, and 40 filler trials for each priming block). Buffer blocks (40 filler trials for each buffer block) only included filler trials and served to neutralize the participants' mindset. Each priming block included 20 different prime pictures, 10 different food-musical instrument pairs, and 10 different neutral-neutral pairs. Each buffer block 
included 5 different neutral prime pictures and 10 different neutral-neutral pairs. The buffer blocks were presented between two priming blocks to reduce the interference from the prime pictures of the previous block. The order of the priming blocks was counterbalanced across participants.

Stimuli in the food dot-probe task. Most of the palatable food stimuli and musical instrument stimuli in the current study were from Werthmann et al. (2011). All image pairs were subjectively matched as closely as possible with regard to the shape, color, brightness, and size of the depicted object. Each picture appeared equally often on the left and on the right side of the screen. All the food stimuli were rated by participants in the final online questionnaire according to how much they like the food.

Prime pictures. 90 candidate prime pictures, with 30 depicting eating enjoyment context (e.g., wedding), 30 depicting health-related context (e.g., sports), and 30 depicting food-unrelated context (e.g., street view) were rated on how much food indulgence and how many healthy food choices they elicited on a 100-mm VAS by asking "How much would you like to indulge in tasty food after viewing the above picture?", and "How inclined are you to choose healthy food after viewing the above picture?", with 0 reflecting 'not at all' and 100 reflecting 'very much'. Ratings were provided by 37 women $\left(M_{\text {age }}=21, S D\right.$ age $=3.20$; no participants of the current study). The 20 eating enjoyment-related pictures with the highest ratings on food indulgence were selected as the hedonic primes; the 20 health-related pictures with the highest ratings on healthy food choices were selected as the health primes; the 20 food-unrelated pictures, with lower ratings on both food indulgence (hedonic primes vs neutral primes: $t(19)=41.26, p<.001$ ) and healthy food choices (healthy primes vs neutral primes: $t(19)=21.49, p<.001)$ were selected as the neutral primes. The prime pictures in the buffer blocks were landscape pictures. For the average priming pictures rating scores per priming condition, see Table 2.

Table 2. Priming Pictures Rating Scores Per Priming Condition.

\begin{tabular}{|c|c|c|c|c|c|c|}
\hline \multirow[b]{2}{*}{ Variables } & \multicolumn{2}{|c|}{ Hedonic primes } & \multicolumn{2}{|c|}{ Health primes } & \multicolumn{2}{|c|}{ Neutral primes } \\
\hline & $M$ & $S D$ & $M$ & $S D$ & $M$ & $S D$ \\
\hline Indulgence & 64.24 & 2.32 & 36.05 & 2.60 & 37.59 & 2.17 \\
\hline Healthy food choices & 39.58 & 6.15 & 60.33 & 3.21 & 36.57 & 4.11 \\
\hline
\end{tabular}

\section{Eye Movement Measurements}


Participant's eye-movement data was collected via a desktop mounted EyeLink 1000 system. All stimuli were presented on a 24 -inch computer screen at a viewing distance of about 57 cm. Using DataViewer software (SR Research, Canada), saccades and fixations were extracted. The display screen was divided into three interest areas: the middle section (represented the location of the fixation cross), and the left and right section (represented the location of the stimuli). The width of the middle-interest area was decided by a given visual angle:2 horizontal degrees (Amir, Zvielli, \& Bernstein, 2016). Fixations located in the middle-interest area, and fixation durations below $60 \mathrm{~ms}$ were discarded (Amir et al., 2016).

\section{Manipulation Check}

The manipulation check was conducted at the end of the experiment, to test whether the three types of prime pictures influenced participants' desire to indulge in palatable foods differently. All prime pictures used in the task were successively and randomly displayed on the screen in three blocks with the same category of prime pictures in one block. Each picture was presented for $1000 \mathrm{~ms}$ (same display time as in the food dot-probe task). After viewing one block, participants were asked to indicate how much they would like to indulge in tasty food right now on a 100-mm VAS. The three blocks were presented in random order.

\section{Procedure}

All participants were tested individually in a laboratory at Maastricht University after $10 \mathrm{am}$. The order of the priming context was counterbalanced across participants. First, the participant's hunger level was measured on a 100-mm VAS together with several filler questions $^{3}$ via a Qualtrics online survey. Then after a 9-point calibration (calibration for proper gaze recording by the system) with subsequent validation (validation for gaze position accuracy achieved by the current calibration) procedure, the food dot-probe task was administered. Next, the manipulation check, the RS, liking of the food pictures, and selfreported height and weight were assessed in another Qualtrics online survey. Finally, we

\footnotetext{
${ }^{3}$ Filler questions: How many glasses of alcohol did you drink yesterday?; How many cups of coffee did you drink yesterday?; How many cigarettes did you smoke yesterday?; How many cups of water did you drink yesterday?
} 
explained the real purpose of the current study to the participant, and the participant was thanked for her participation and received a small reward (either 1.5 hour-course credits or 10 euro in gift-voucher). The whole procedure lasted about 1.5 hours.

\section{Data Reduction and Analysis}

All AB scores were computed separately for each priming condition for each participant.

\section{Reaction Time-Based Attentional Bias for Food}

Mean AB for food was calculated by subtracting the mean RTs of CTs from the mean RTs of ITs. So, a positive value reflects an $\mathrm{AB}$ towards food, and a negative value an $\mathrm{AB}$ away from food. To obtain sequential TL-BSs, first, each CT was paired with an IT that was as close as possible in time and no further than five trials away. Next, the CT in each pair was subtracted from the IT in that pair. TL-BS variability for food was computed using the sum of absolute distances between sequential TL-BSs divided by the total number of TL-BSs (Zvielli et al., 2015).

\section{Eye Movement-Based Attentional Bias for Food}

Based on eye-movement data, two average AB for food scores were computed: mean initial fixation duration bias on food and mean dwell time bias on food. The initial fixation duration represents the duration of the first fixation that remains on one of the picture stimuli. Initial fixation durations were firstly averaged across trials per participant, separately for the two categories (food/non-food). Then, per participant, the mean initial fixation duration bias on food was calculated by subtracting the averaged initial fixation duration in the interest area containing a musical instrument stimulus from the averaged initial fixation duration in the interest area containing a food stimulus.

The dwell time is the total time that a gaze remained at each stimulus during the $2000 \mathrm{~ms}$ presentation time. Mean dwell time bias was calculated by subtracting the mean dwell time in the interest area with a musical instrument stimulus from the mean dwell time in the interest area with a food stimulus. So, positive values reflect relatively more attention for food than for musical instruments. The EM-based dynamic changes of $\mathrm{AB}$ for food were operationalized as the standard deviation $(S D)$ of the initial fixation duration bias on food, 
the $S D$ of dwell time bias on food, and as the number of switches between the food and nonfood stimulus within each trial.

\section{Data reduction}

Firstly, buffer blocks were excluded from analyses, and then trials were excluded from analyses if they contained error responses, were faster than $200 \mathrm{~ms}$, slower than $2000 \mathrm{~ms}$, and after that if they deviated more or less than three SDs from each participant's mean RT (Werthmann et al, 2011; 2.20\% of the RT data were excluded). In addition, it was checked if participants moved their eyes on a sufficient proportion (50\%) of trials (Bradley, Mogg, \& Millar, 2000), and this led to no participant exclusion. Then both RT-based and EM-based $\mathrm{AB}$ scores per priming context were calculated separately, after that under each priming context, it was checked whether any $\mathrm{AB}$ score deviated more or less than three SDs from the respective mean of the whole sample (outliers). Finally, 29 outlier AB scores (the percentage of these outliers was $1.62 \%$ ) were replaced by the respective nearest score of the whole sample (Wilcox, 2001) ${ }^{4}$.

\section{Analysis plan}

First, the manipulation check regarding priming context was conducted by comparing the scores on the manipulation question between hedonic/health context and neutral context in a repeated-measures of ANOVA. Then, two repeated-measures of MANOVAs were conducted to test the effects of different priming contexts and the interaction between priming contexts and dietary restraint (mean-centered) on both AB fluctuation scores (TL-BS variability, SD of initial fixation duration bias, SD of dwell time bias, and number of switches between the food and nonfood stimulus) and mean $\mathrm{AB}$ scores (mean $\mathrm{AB}$ scores, mean initial fixation duration bias, and mean dwell time bias). According to previous studies (e.g., Hardman et al., 2020; Tapper, Pothos, \& Lawrence, 2010), BMI, hunger level, and food liking scores might influence $\mathrm{AB}$ scores, last, correlations between $\mathrm{AB}$ fluctuation scores/mean $\mathrm{AB}$ scores and

\footnotetext{
${ }^{4}$ The analyses with unchanged values or exclusion of these values showed the same pattern of results and led to the same conclusions.
} 
these variables were conducted as exploratory analyses. Because of the large number of statistical tests, alphas were adjusted using the Bonferroni method.

\section{Results}

\section{Manipulation Check}

Data were analyzed in a repeated-measures ANOVA with priming condition as the factor, and scores on the manipulation check question "how much would you like to indulge in tasty food after viewing the above video" as the dependent variable. Scores on the manipulation check question differed significantly between priming conditions, $F(2,168)=83.50, p<.001$. Post hoc tests using the Bonferroni correction (adjusted alpha $=0.025$ ) revealed that ratings were higher for the hedonic condition, $M=63.88, S D=2.80$, than for both the health, $M=$ $36.44, S D=2.71$, and the neutral condition, $M=35.04, S D=2.84$; $p s<.001$. The health condition did not differ significantly from the neutral condition, $p=1.00$. So, the hedonic context was successfully induced, but the health context was not.

\section{Average Performance on Tasks}

Participants' average performance on the task is displayed in Table 3.

Table 3. Measures of AB Per Priming Condition.

\begin{tabular}{|c|c|c|c|c|c|c|}
\hline \multirow{2}{*}{ variables } & \multicolumn{2}{|c|}{ Hedonic Priming } & \multicolumn{2}{|c|}{ Healthy Priming } & \multicolumn{2}{|c|}{ Neutral Priming } \\
\hline & $M$ & $S D$ & $M$ & $S D$ & $M$ & $S D$ \\
\hline TL-BS variability & 132.15 & 49.65 & 128.77 & 46.88 & 136.74 & 55.97 \\
\hline Mean AB & 9.64 & 25.75 & 7.83 & 24.54 & 8.83 & 25.65 \\
\hline Mean initial fixation duration bias & 39.08 & 73.51 & 35.98 & 67.11 & 46.39 & 73.26 \\
\hline Mean dwell time bias & 48.80 & 295.95 & 15.56 & 242.53 & 50.89 & 211.06 \\
\hline SD of initial fixation duration bias & 359.02 & 152.65 & 375.95 & 173.08 & 376.39 & 186.10 \\
\hline SD of dwell time bias & 894.42 & 345.52 & 911.58 & 373.72 & 894.07 & 363.02 \\
\hline Number of switches & 1.84 & 0.54 & 1.80 & 0.66 & 1.83 & 0.59 \\
\hline
\end{tabular}


Note. TL-BS: trial-level bias scores; AB: attentional bias; SD: standard deviation.

\section{Effects of priming condition and restrained eating on attentional bias for food}

\section{Mean Attentional Bias for food}

The results from MANOVA showed that there was no statistically significant main effect of priming contexts, $F(6,78)=0.81, p=.57$, Wilks' $\Lambda=.94$, partial $\eta^{2}=.06$, on the combined dependent variables, mean $\mathrm{AB}$ scores. The interaction effect between priming contexts and dietary restraint on the combined dependent variables was also not statistically significant, $F(6,78)=1.87, p=.10$, Wilks' $\Lambda=.87$, partial $\eta^{2}=.13$.

\section{Fluctuations in Attentional Bias for food}

There was no statistically significant difference between the priming contexts on the combined dependent variables, $\mathrm{AB}$ fluctuation scores, $F(8,76)=1.20, p=.31$, Wilks' $\Lambda=.89$, partial $\eta^{2}=.11$. The interaction effect between priming contexts and dietary restraint on the combined dependent variables was also not statistically significant, $F(8,76)=0.73, p=.66$, Wilks' $\Lambda=.93$, partial $\eta^{2}=.07$.

\section{Correlational analyses}

\section{Mean Attentional Bias for food}

The results of the correlations (adjusted alpha $=0.0167$ ) showed that food liking was positively related to mean initial fixation duration bias and mean dwell time bias. Apart from that, BMI, hunger level, and food liking were not related to any other mean measures of $\mathrm{AB}$ scores, see Table 4.

\section{Fluctuations in Attentional Bias for food}

As for the AB fluctuation scores, only BMI was significantly related to the TL-BS variability: participants with a higher BMI showed more variability in AB for food (adjusted alpha = 0.0125). For more information, see Table 5. 
Table 4. Correlations Between BMI, Hunger Level, Food Liking, and Mean AB Indexes.

\begin{tabular}{lllllll}
\hline$N=85$ & 1 & 2 & 3 & 4 & 5 & 6 \\
\hline 1.Mean AB & 1 & $.35^{*}$ & $.50^{*}$ & .001 & -.03 & .21 \\
2.Mean initial fixation duration bias & & 1 & $.85^{*}$ & -.0003 & .25 & $.35^{*}$ \\
3.Mean dwell time bias & & 1 & .01 & .19 & $.46^{*}$ \\
4.BMI & & & 1 & -.08 & -.23 \\
5.Hunger & & & & 1 & $.29^{*}$ \\
6.Food liking & & & & & 1 \\
\hline
\end{tabular}

Note. AB: attentional bias; BMI: body mass index; hunger: hunger level of participants (0100 VAS); food liking: average food liking score for the high-calorie food stimuli presented in the food-dot probe task (0-100 VAS); ${ }^{*} p<.0167$.

Table 5. Correlations Between BMI, Hunger Level, Food Liking, and AB Fluctuation Indexes.

\begin{tabular}{llllllll}
\hline$N=85$ & 1 & 2 & 3 & 4 & 5 & 6 & 7 \\
\hline 1. TL-BS variability & 1 & -.12 & -.12 & .02 & $.27^{*}$ & -.0002 & .14 \\
2.SD of initial fixation duration bias & & 1 & $.66^{*}$ & $-.57^{*}$ & .001 & .14 & .13 \\
3.SD of dwell time bias & & & & $-.65^{*}$ & -.06 & .05 & .10 \\
4.Number of switches & & & 1 & .07 & -.11 & -.17 \\
5.BMI & & & & 1 & -.08 & -.23 \\
6.Hunger & & & & & & \\
7.Food liking & & & & & & & \\
\end{tabular}

Note. TL-BS: trial-level bias scores; SD: standard deviation; BMI: body mass index; hunger: hunger level of participants (0-100 VAS); food liking: average food liking score for the highcalorie food stimuli presented in the food-dot probe task $(0-100 \mathrm{VAS}){ }^{*} p<.0125$. 


\section{Discussion}

This study examined whether hedonic and health priming conditions influence AB for food, and how this effect would be moderated by dietary restraint. Both reaction time (RT) -based and eye movements (EMs)-based mean tendencies and variability of attentional bias (AB) for high-calorie foods were measured. Unexpectedly, it was found that both the priming condition and the interaction between the priming condition and dietary restraint did not significantly affect AB for food. However, BMI was positively related to TL-BS variability, which is in line with previous studies (Liu et al., 2019a, b). In addition, food liking was positively related to initial fixation duration bias and dwell time bias. So, participants who reported higher liking of the presented food stimuli looked at the food stimuli longer.

The results of the manipulation check demonstrated that after experiencing hedonic priming pictures, participants reported that they wanted to indulge in high-calorie foods more as compared to both the neutral and the health priming context, which means the hedonic priming context indeed induced a hedonic goal. However, the priming manipulation was not entirely successful, as there was no significant difference between the health and neutral priming context. Thus, it can be concluded that even though the hedonic priming was successful, it did not translate to effects on $\mathrm{AB}$ for food. It contradicts a previous study (Werthmann et al., 2016), which found that a health mindset as compared to a palatability mindset decreased RT-based AB for high-calorie foods in participants with higher dietary restraint. However, it should be noted that in Werthmann et al. (2016), the two mindset conditions (health vs hedonic) only differed significantly on the rated importance of health, but not on the rated importance of palatability, and the design did not include a neutral mindset condition. So, less AB for high-calorie foods in a health mindset than in a palatability mindset should likely be attributed to an increased importance of health in the health mindset condition. In addition, to the best of our knowledge, previous study only found a significant influence of context on $\mathrm{AB}$ for food primed participants with a dieting-related context instead of a hedonic context (van der Laan et al., 2017). So, maybe health/weight concerns more easily reduce $\mathrm{AB}$ for high caloric foods than that a hedonic focus increases $\mathrm{AB}$ for food. Therefore, the unsuccessful manipulation in the health condition in our study might explain why our results are not in line with previous studies. To induce weight concern, more salient cues should be included in future studies, like the scales and weight/dieting-related 
information instead of the exercise and healthy food pictures used in the current study. It also suggests that mild health cues in real life might not be enough to influence attention, especially when hedonic cues are presented at the same time. Morerover, the current study used a within-subjects design, whereas previous studies (e.g., Werthmann et al., 2016) used a between-subjects design. So, it might be that the buffer blocks were not sufficient to avoid spill-over between the different priming conditions. The current study only investigated the effect of context priming on $\mathrm{AB}$ for palatable foods. It would be interesting to investigate how different contexts affect $\mathrm{AB}$ for healthy food stimuli in future studies.

Dietary restraint and BMI are frequently believed to be related to $\mathrm{AB}$ for high caloric palatable food and this has been tested in many studies (e.g., Castellanos et al., 2009; Meule et al., 2012). However, the empirical evidence does not support this claim (e.g., Doolan et al., 2015; Hagan et al., 2020; Hardman et al., 2020; Roefs et al., 2015; Werthmann et al., 2015). The current study also did not find any significant relationship between dietary restraint or $\mathrm{BMI}$ and mean $\mathrm{AB}$ for food, not even in the hedonic priming condition. So, the results from the current study question the notion that $\mathrm{AB}$ for high-caloric palatable food is a trait-like feature of people with a high BMI or scoring high on dietary restraint.

Interestingly, the current study did show a positive association between BMI and TL-BS variability for food, which is in accordance with previous studies (Liu et al., 2019a, 2019b), which included a range of participants (normal-weight females and children, but also included normal-weight children, overweight/obese children, and overweight females). Notably, in one of these studies (Liu et al., 2019b) it was found that this relation between BMI and TL-BS variability does not hold if attention control is high and another study directly found that the relationship between anxiety and the TL-BS variability for anxietyrelated stimuli was significantly mediated by attention control (Clarke et al., 2020). Therefore, the variability of $\mathrm{AB}$ for food might reflect weaker executive control. In detail, weaker executive control might make people less likely to have a consistent, prolonged attention to food stimuli during the task, therefore, causing more variability in AB for food.

The current study also found positive associations between participants' food liking and EM-based mean AB for food (both mean initial fixation duration bias and mean dwell time bias), which is consistent with previous studies (e.g., Kemps \& Tiggemann, 2009). The 
incentive salience model (Berridge, 2009) proposed that $A B$ for food reflects appetitive motivation, and except for the influence of momentary motivation on $\mathrm{AB}$ for food, the relatively stable trait, food liking, should be also closely related to AB for food. However, the average food liking score was relatively low in the current study, although the food stimuli used in the current study included only widely liked food items, like chocolate, and crisps. Tailoring food stimuli to the individual participant should be considered in future studies.

Except for the within-subject design and relatively low food liking score, there are several other limitations of the current study that need to be mentioned here. First, the participants included in this study were fairly homogeneous. They are all females and most of them were students. Future studies are needed to confirm the effect of context priming on $\mathrm{AB}$ for food in diverse populations. Second, because BMI is not our main focus, we used self-reported BMI instead of measure it, which could have caused inaccuracy and therefore have affected the results regarding to BMI.

\section{Conclusion}

The present study assessed the effect of context on $\mathrm{AB}$ for food. The situational context was manipulated by priming participants with hedonic, healthy, and neutral pictures. Contrary to our hypothesis, the current results did not provide evidence for the influence of hedonic or health-related context on individuals' AB for food. A positive association between BMI and TL-BS variability for food was found, which replicates results from previous studies (Liu et al., 2019a; Liu et al., 2019b). Food liking was positively related to AB for food, which is in line with the idea that $\mathrm{AB}$ for food reflects appetitive motivation (Field et al., 2016). Finally, adding to the inconsistency in the field (e.g., Doolan et al., 2015; Hagan et al., 2020; Hardman et al., 2020; Roefs et al., 2015; Werthmann et al., 2015), this study failed to show any significant relationship between dietary restraint or BMI and average AB for food. 
Chapter 4

Dynamics of Attentional Bias for Food in Dutch and Chinese Children and the Role of Executive Control

Published as:

Liu, Y., Nederkoorn, C., \& Roefs, A. (2019). Dynamics of attentional bias for food in Dutch and Chinese children and the role of executive control. Appetite, 143, 104421. https://doi.org/10.1016/j.appet.2019.104421 


\section{Abstract}

The current study examined the relationships among dynamics of attentional bias for food, executive control and adjusted BMI\% among children. 106 Dutch (8-12 years old, 56.60\% female) and 99 Chinese children (9-13 years old, $44.44 \%$ female) participated in the study. All participants first completed a self-report hunger questionnaire, and then the food dotprobe task and attention network task were administered. Both mean attentional bias and the variability of attentional bias were computed as people often experience the opposite goals to approach and avoid palatable food. We hypothesized that only the variability of attentional bias for food would be positively related to adjusted BMI\%. In addition, such association would be moderated by the executive control, which would be also negatively related to adjusted $\mathrm{BMI} \%$. The results showed that adjusted BMI\% was only positively associated with the variability of attentional bias for food in Dutch children. However, such association did not exist in Chinese children and executive control was not a significant moderator of the relation between the variability of attentional bias for food and adjusted BMI\% in both Dutch and Chinese children. Unexpected, compared with Dutch children, Chinese children had stronger executive control, which was directly related to a lower adjusted BMI\%. This study emphasized both TL-BS variability for food and executive control might have an effect on adjusted $\mathrm{BMI} \%$, in addition, culture possibly serve as a reason for the inconsistent results between Dutch and Chinese children.

Keywords: attentional bias; variability; executive control; children 


\section{Introduction}

Worldwide, over 340 million children and adolescents were overweight or obesity in 2016 (WHO, 2016). It is well known that a prolonged positive energy imbalance, that is, consuming more calories than expending, to obesity and overweight (Wood, 2006), but sticking to a healthy diet and exercise plan is difficult for most people. We live in an "obesogenic" environment, in which we are surrounded by energy-dense palatable foods (Hill \& Peters, 1998), serving as potential reward cues (Berthoud, Lenard, \& Shin, 2011). According to incentive salience theory of addiction (Berridge, 1996; Berridge, 2009), reward-related cues, like palatable foods, can trigger incentive salience to the representations, which modify dopamine-related systems and make that stimulus even more pleasant, attention-grabbing, and finally affect eating behavior.

As attentional bias $(\mathrm{AB})$ for food is considered one of the potential vulnerability factors contributing to overeating and obesity (e.g., Kemps, Tiggemann, \& Hollitt, 2014; Yokum, Ng, \& Stice, 2011), a great deal of research has explored AB for food among different weight groups. However, empirical evidence of food-related AB is contradictory (Field et al., 2016; Roefs et al., 2015). In brief, people with overweight or obesity showed more, equal or less attention for food cues, compared with normal weight participants (e.g., Deluchi, Costa, Friedman, Goncalves, \& Bizarro, 2017; Doolan, Breslin, Hanna, \& Gallagher, 2015; Werthmann et al., 2015; Nummenmaa, Hietanen, Calvo, \& Hyona, 2011; Graham, Hoover, Ceballos, \& Komogortsev, 2011). Another issue is that most of the research focused on $\mathrm{AB}$ for food in western adults and only a few studies included children and eastern individuals in their research. Recent studies found that even in early childhood, children began to experience weight concern (Tatangelo, McCabe, Mellor, \& Mealey, 2016; Damiano, Paxton, Wertheim, McLean, \& Gregg, 2015), and the growth in BMI has accelerated in both east and south Asia (Abarca-Gómez et al., 2017). Thus, research should focus more on the attention pattern for food in children and people from diverse cultures.

The results of the limited number of AB-studies in children are inconsistent as well. For example, in a food dot-probe task (MacLeod, Mathews, \& Tata, 1986) with eye-tracking, Werthmann et al. (2015) found that children with obesity did not show a significantly larger $\mathrm{AB}$ for food pictures than animal pictures than healthy-weight control participants. However, 
Braet and Crombez (2003) did find that children with obesity were slower in naming the color of a colored food word than a colored neutral word in the food Stroop task. Though this food Stroop effect is sometimes interpreted as evidence for AB for food, this type of Stroop task is not often used anymore to assess $\mathrm{AB}$, as it is unclear what cognitive process underlies the effect (Field \& Cox, 2008).

The reason for the inconsistency in the $\mathrm{AB}$ for food literature could be the doublefacetted nature of high-caloric food stimuli (Roefs, Fransen, \& Jansen, 2018). High-caloric foods are craved by many people, but are also a cause of concern for many as these foods can cause weight gain. This might be especially true for people with obesity and for highrestrained eaters (high-restrained eaters is defined as trying to restrict their food intake, but not always succeed in refraining food consumption (Stice, Fisher, \& Lowe, 2004)). Mean $\mathrm{AB}$ score was adopted by almost all studies to express $\mathrm{AB}$ in the dot-probe task, one of the most widely used tasks to acquire participants' AB for certain stimuli (Ataya et al., 2012; Schmukle, 2005). Whereas, mean AB score only provides the central tendency of $\mathrm{AB}$ and conceals the information of $\mathrm{AB}$ at different time points. That is, how $\mathrm{AB}$ fluctuates across trials during the task. Recently, Zvielli et al. (2015) proposed another computing method for the dot-probe task, trial-level bias scores (TL-BS), to express AB across time. It uses all trials to create a sequence of $\mathrm{AB}$ scores. TL-BS variability is then calculated to express the degree of fluctuation in $\mathrm{AB}$ toward and away from the stimuli of interest. Compared with mean $\mathrm{AB}$ score, TL-BS variability could better predict BMI, depression, and spider phobia (Liu, Roefs, Werthmann, Nederkoorn, 2019; Zvielli, Vrijsen, Koster, \& Bernstein, 2016; Zvielli et al., 2015). The advantage of TL-BS is that it can capture the strong opposite motives people may have for food stimuli. The opposite motives can lead to fluctuations in attention, which can be captured with the TL-BS.

Another possible reason for the inconsistent results in the $\mathrm{AB}$ for food literature could difference in executive control (EC) ability in participants. Posner and Petersen (1990) proposed that attention can be divided into three networks: alerting, orienting, and EC. Among them, EC is defined as the ability to resolve the conflict among responses, which can be captured by attention network task (ANT). It was found that only in the low EC group, $\mathrm{AB}$ for alcohol was related to alcohol consumption (van Hemel-Ruiter, de Jong, Ostafin, \& Wiers, 2015). So, it is reasonable to assume that high in EC means that the food-related 
weight gain concern can be activated longer and suppress the hedonic side of food, resulting in fewer alternations in attention for chronic dieters. In this sense, EC may moderate the relationship between TL-BS variability for food and BMI. Specifically, there would be a stronger relationship for TL-BS variability for food and BMI in people with weak EC ability and a weaker relationship for TL-BS variability for food and BMI in people with strong EC ability.

In summary, the current study used the food dot-probe task and the child-version of the attention network task (ANT) (Rueda et al., 2004; Fan, McCandliss, Sommer, Raz, \& Posner 2002) to explore the relationships among AB for food, EC and BMI in Dutch and Chinese children. In addition to mean AB for food, TL-BS variability was computed to describe the dynamic changes of $\mathrm{AB}$ for food. To test if a possible relation between TL-BS variability and BMI is specific for food, or would be observed for other types of attractive stimuli as well, cute animal stimuli were included in addition to the food and neutral stimuli. Study 1 was conducted in a Dutch population and study 2 in a Chinese population.

We hypothesized that (1) TL-BS variability for food stimuli but not for cute animal stimuli would be significantly related to BMI (2) EC ability would be negatively related to BMI; (3) EC would moderate the relationship between TL-BS variability for food and BMI: we expected that in people with less EC, the relation between TL-BS variability for food and BMI would be stronger. Note that the current study was pre-registered and can be found at AsPredicted (http://aspredicted.org/blind.php? $\mathrm{x}=\mathrm{j} 833 \mathrm{ez}$ ).

\section{Study 1}

In Study 1, we investigated the performance of Dutch children in a food dot-probe task and the child-version of the ANT.

\section{Methods}

\section{Participants}

Because no study has tested the relations among the variability of AB, EC, and BMI, it was not possible to obtain a reliable estimate of the to be expected effect size. Therefore, according to Cohen (1988), we used a conventional medium effect size $\left(f^{2}=0.15\right)$ to roughly 
calculate the required sample sizes in our two studies. To test our main hypothesis, a regression analysis was conducted, with 5 predictors $\left(f^{2}=.15\right.$, alpha $=.05$, power $\left.=.80\right)$, requiring at least 92 participants in each study. 107 Dutch children across the weight spectrum were recruited from a local primary school and several after-school child-care centers in the south of Netherlands. One child was excluded from the analysis of AB and six children were excluded from the analysis of EC because of poor performance on the food dot-probe task or ANT (> 25\% mistakes; one child made too many mistakes on both tasks). In addition, three children were excluded from the analysis of EC because of missing data. Finally, 106 children provided AB data $\left(99.07 \%\right.$ of the original sample; $M_{\text {age }}=10$ years old, $S D_{\text {age }}=1.00$, Range $_{\text {age }}=8-12$ years old $)$ and 98 (91.59\% of the original sample) children provided EC data. For Study 1 and Study 2, ethical approval was obtained from the ethics committee of the Faculty of Psychology \& Neuroscience, Maastricht University, and schools/caregivers provided consent for their children to join in the current study.

\section{Materials}

Hunger Questionnaire. Participants' hunger level was assessed on a 10-point scale, by asking "How hungry are you right now?", with 0 indicating absolutely not hungry at all, and 10 indicating extremely hungry.

Food Dot-Probe Task. AB was assessed by the food dot-probe task (MacLeod, Mathews, \& Tata, 1986).

Trial Procedure. In this task, each trial started with a fixation cross presented in the middle of the screen for $500 \mathrm{~ms}$. Subsequently, two pictures were simultaneously presented side-by-side for a duration of $500 \mathrm{~ms}$. Next, one of the pictures was replaced by a probe $\left(^{*}\right)$, which randomly appeared on the left or right side of the screen, and the participant needed to indicate the location of the probe by a button-press. The probe was presented until the response or for a maximum of $2000 \mathrm{~ms}$. Participants were instructed to focus their gaze first on the central fixation cross and then respond to the probe as quickly as possible.

Trial Types: The task included five different types of trials: food-incongruent trials (food IT), food-congruent trials (food CT), animal IT, animal CT, and neutral-neutral trials. On the food trials, a food and a neutral picture were presented, whereas on animal trials, an 
animal and a neutral picture were presented. During ITs, the probe appeared in the location of the neutral picture, whereas during CTs, the probe appeared in the location of the target stimulus (food or animal). Neutral trials included two neutral stimuli (non-food/animal pictures).

Number of Trials. Trials were evenly and randomly distributed across 4 blocks, 288 trials in total. Food-neutral trials consisted of 12 pairs of palatable food (target) and neutral stimuli; animal-neutral trials consisted of 12 pairs of animals (target) and neutral stimuli, and the neutral trials consisted of 12 paired neutral stimuli. Each of the paired stimuli was presented 8 times, with the target stimuli appearing on the left of the screen for 4 times and on the right side of the screen for another four times. Equal numbers of CTs and ITs were presented.

The palatable food stimuli and animal stimuli used in Study 1 were taken from Werthmann et al. (2015), and were rated as the most attractive pictures by children. The neutral stimuli were chosen to be as neutral as possible (e.g., box, shoes, hanger). All image pairs were subjectively matched by experimenters as closely as possible with regard to the shape, color, brightness, and size of the depicted object.

Child-version Attention Network Task (ANT). The ANT is a combination of a precueing task (Posner, 1980) and a flanker task (Eriksen \& Eriksen, 1974). In the child-version of the ANT (Rueda et al., 2004; Fan et al., 2002), each trial started with a central fixation cross, and after a random time interval between 400 and $1600 \mathrm{~ms}$, one of the cues appeared above, below or at the location of the fixation cross for $100 \mathrm{~ms}$. Then the target (central yellow fish) together with flanker or the target alone appeared above or below the central fixation cross after $400 \mathrm{~ms}$. Participants were instructed to determine whether the target was pointing to the left or to the right by pressing the corresponding key. The target presented on the screen until response, with a maximum of 1700ms, see Figure 1.

There were four different types of cue (no cue, central cue, double cue, and spatial cue), and three different types of flankers (congruent, incongruent and neutral) in this task. In the central cue condition, a dot appeared at the location of the fixation cross; in the double cue condition, two dots appeared above and below the fixation cross simultaneously; in the spatial cue condition, a dot appeared in the location of the upcoming target. For the flanker, 
in the congruent trials, the flanking fishes were pointing in the same direction as the middle target fish; on the incongruent trials, the flanking fishes were pointing in the opposite direction as the middle target fish; and on the neutral trials, there were no flankers and the middle target fish appeared lonely. The task consisted of 24 practice trials and 3 experimental blocks of 48 trials in each block. Feedback on performance was only available during the practice.

Figure 1. An example of the ANT.

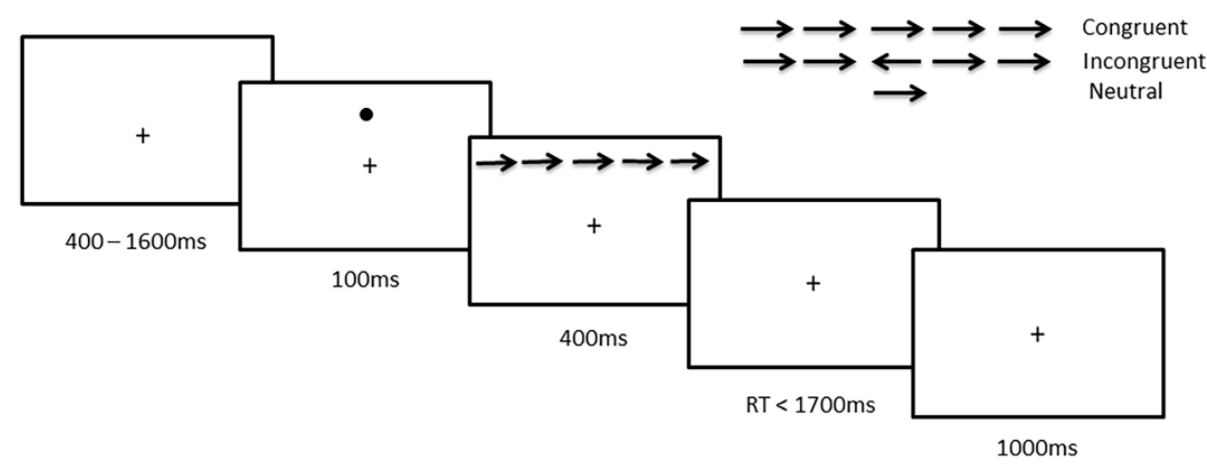

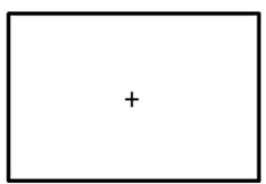

No cue

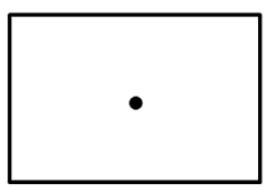

Central cue

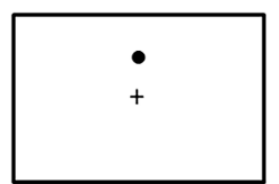

Spatial cue

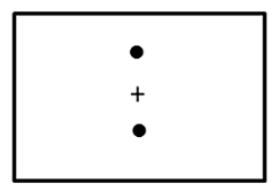

Double cue

\section{Procedure}

All children were tested in groups (fewer than 10 children per group) in their schools on a laptop. Children firstly completed a questionnaire to assess their basic information and hunger. Then, the dot-probe task and ANT were administered. The order of these two tasks was counterbalanced across participants. Participants could take a short break between each test block and at the end of the first task. Finally, their weight and height were measured to obtain the adjusted BMI\% (BMI divided by the national (Dutch) norm BMI (Van Winckel $\&$ Van Mil, 2001), adjusted for sex and age, $\times 100$.). To make sure all children understood the instructions of both the food dot-probe task and the ANT task, the experimenter firstly 
explained both tasks to the children, reading from a standard script. Then, the children were asked to read the instruction again. After that, two short practice blocks were conducted before starting the real tasks, during which the experimenter checked if the child performed the task correctly. In addition, before and after the practice blocks of both the food dot-probe task and the ANT task, children were told that they could ask for help if they did not understand the instructions or had other questions. The mean performance rates were good in both studies (for Dutch children, mean correct rates of the dot-probe task and the ANT task were $94 \%$ and $93 \%$ respectively; for Chinese children, they were $97 \%$ and $94 \%$ respectively), indicating that they understood the task. The whole procedure lasted about forty minutes.

\section{Data Reduction and Analysis}

Data Reduction. Reaction times (RT) in the food dot-probe task and ANT were excluded from further analyses if they were related to error responses, were faster than $200 \mathrm{~ms}$, or deviated more than $3 S D$ s from each participant's mean RT (after excluding error responses and faster than $200 \mathrm{~ms}$ trials). Therefore, $6.27 \%$ of trials from the food dot-probe task and $7.08 \%$ of trials from the ANT were discarded. In addition, two outliers (4.25 SD and $4.30 S D$ above the mean value) in the adjusted BMI\%, one outlier (3.92 SD above the mean value) in the TL-BS variability for animal, and one outlier (4.60 SD above the mean value) in EC were replaced by the nearest score of the whole sample (see Wilcox, 2001) .

The Computation of Attentional Indexes. Mean $\mathrm{AB}$ scores for food and animal were calculated separately by subtracting the mean RT of congruent from the mean RT of corresponding incongruent trials. To acquire the trial-level bias scores (TL-BS) for both food and animal conditions, we paired each congruent trial with an incongruent trial that was as close as possible in time, no further than five trials away within the same condition. A positive bias score indicated attention toward food or animal and a negative bias score indicated attention away from food or animal. The variability of AB (TL-BS variability) for food and animal were calculated using the sum of absolute distances between sequential TL-

\footnotetext{
5 The analyses with unchanged values or exclusion of these values showed the same pattern of results and led to the same conclusions.
} 
BSs on food or animal condition divided by the total number of TL-BSs (Zvielli et al., 2015). For the neutral condition, congruent and incongruent trials were randomly assigned, and the TL-BS variability for neutral-neutral trials was calculated with the same approach as TL-BS variability for food and animal. The mean number of IT-CT pairs was $52.33(S D=4.63)$ for the food condition, $52.48(S D=4.41)$ for the animal condition, and $52.49(S D=5.04)$ for the neutral condition; the mean distance between each IT and CT was 2.57 trials $(S D=0.19)$ for the food condition, $2.55(S D=0.19)$ for the animal condition, and $2.58(S D=0.20)$ for the neutral condition. According to Rueda et al. (2004), EC was calculated by subtracting the median RT of all the congruent trials in the ANT from the median RT of all the incongruent trials. A lower score indicates better EC ability.

Analysis Plan. First, correlations were computed to explore the relationships among adjusted $\mathrm{BMI} \%$, mean $\mathrm{AB}$ scores, TL-BS variability, and EC and to test whether the control variables hunger level and age should be added as confounders in the regression analysis. According to Gruijters (2016), potential confounders should be controlled for if they are related to the dependent variable. Then, a one-sample $t$-test against zero was used to test whether an $\mathrm{AB}$ for food exists in Dutch children. Afterwards, hierarchical linear regressions were performed to investigate predictors of adjusted BMI\% after controlling for potential confounders. Finally, the moderating effects of EC on the relation between TL-BS variability for food and adjusted $\mathrm{BMI} \%$ and on the relation between mean $\mathrm{AB}$ scores for food and adjusted BMI\% were examined with the "process module" in SPSS.

\section{Results}

Demographic Information and Average Performance on Tasks

Demographic information and average performance on tasks of participants in Study 1 can be seen in Table 1. 


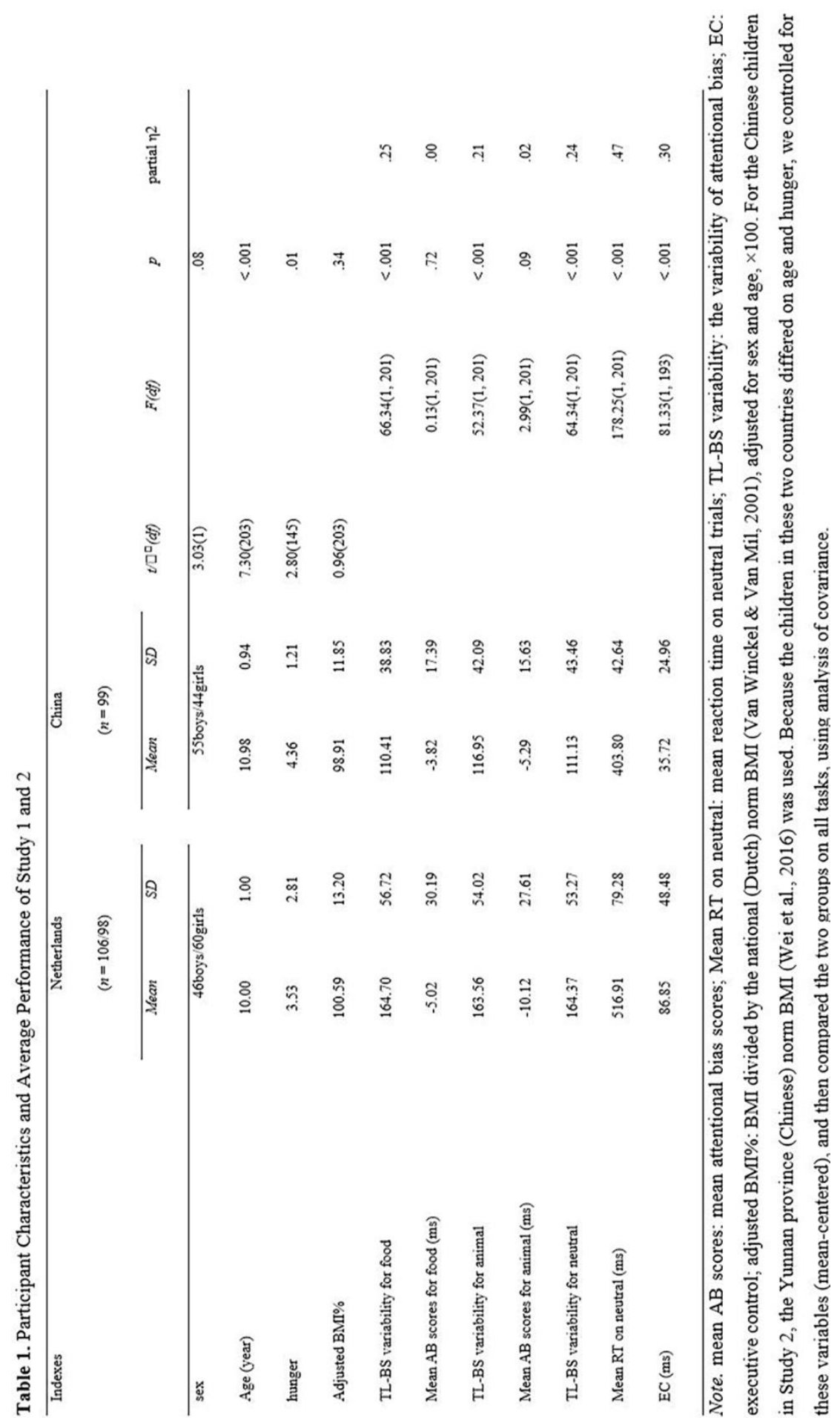




\section{Correlations}

The results of zero-order correlations showed that only TL-BS variability for food was positively related to adjusted $\mathrm{BMI} \%$. However, mean $\mathrm{AB}$ scores for food was marginally negatively related to adjusted $\mathrm{BMI} \%$, suggesting a decrease in $\mathrm{AB}$ for food with increasing BMI. All TL-BS variability variables (i.e., food, animal, neutral) and mean RT on neutral trials were positively related to the level of hunger: hungrier children showed more variability and slower RT. Moreover, children showed less variability of AB (TL-BS variability) for food, animal and neutral pictures with increasing age.

\section{Mean $A B$ for food in the Sample}

The result of the one-sample $t$-tests indicated that Dutch children showed a significant attentional avoidance of food stimuli $(\mathrm{t}(98)=2.19, \mathrm{p}<.05)$.

\section{Hierarchical Linear Regressions}

Because none of the potential confounders were related to adjusted BMI\%, there was no need to control for them (see Table 2). We only added the predictors that were at least marginally significantly $(p<.10)$ correlated with the outcome variable, adjusted BMI\%, to the regression model. Mean AB scores for food was added in the first step and TL-BS variability for food in the second step. The results showed that the model including only mean $\mathrm{AB}$ scores for food marginally significant explain variance in adjusted BMI\% but the effect was in the opposite direction, $F(1,104)=3.61, p=.06$. However, introducing TL-BS variability for food significantly improved the entire model, $F(2,103)=3.92, p<.05$, and explained significant additional variance in adjusted $\mathrm{BMI} \%$. More details can be found in Table 3. 


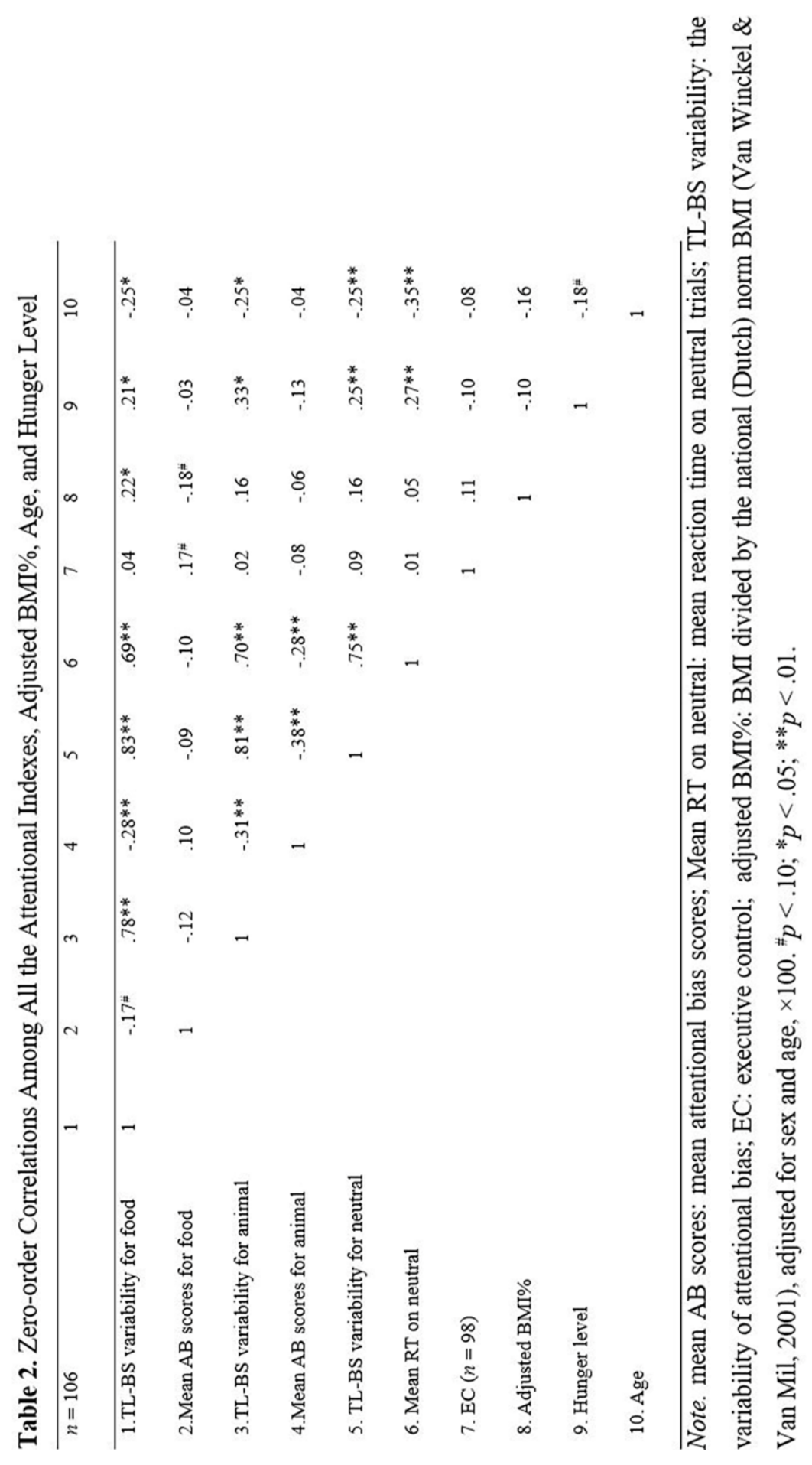




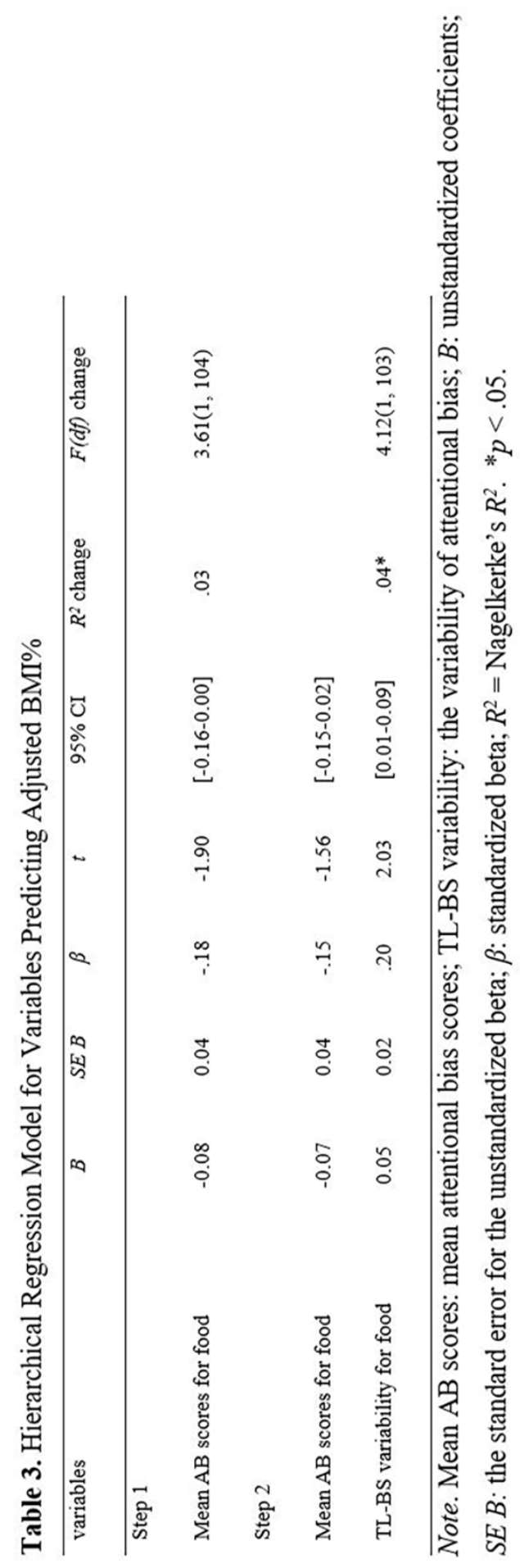

Page | 78 


\section{The Moderating Role of Executive Control}

Although adjusted $\mathrm{BMI} \%$ was marginally related to mean $\mathrm{AB}$ scores for food, the effect was in the opposite direction, we only conducted a moderation analysis to examine the moderating role of EC in the relationship between TL-BS variability for food and adjusted $\mathrm{BMI} \%$. The independent and moderator variables were mean-centered first. Adjusted BMI\% was added as a dependent variable, TL-BS variability for food as an independent variable, and EC as a moderator. The results showed that the interaction term between EC and TL-BS variability for food did not explain significant variance in adjusted $\mathrm{BMI} \%, \Delta R^{2}=.02, F(1$, $94)=1.73, p=.19$.

\section{Discussion}

In the first study, we found that among all the attention variables (the variabilities of attentional bias (AB), mean $\mathrm{AB}$ scores, and executive control (EC)), only the variability of AB (TL-BS variability) for food was significantly related to adjusted BMI\% in Dutch children. After controlling for mean $\mathrm{AB}$ for food, this relationship remained significant. Mean AB scores for food was marginally negatively associated with adjusted BMI\% in Dutch children and Dutch children expressed significant attentional avoidance of food stimuli. Moreover, EC was not a significant moderator of the relation between the variability of $\mathrm{AB}$ for food and adjusted $\mathrm{BMI} \%$. Overall, these results are partly consistent with our hypothesis that children's $\mathrm{AB}$ for food is dynamic and that more variability of $\mathrm{AB}$ for food is related to a higher BMI. Together with the inconsistent results of mean AB for food among people with overweight or obesity in previous studies (e.g., Roefs et al., 2015; Werthmann, Jansen, \& Roefs, 2015), the marginally significant negative association we found between mean $\mathrm{AB}$ scores for food and adjusted $\mathrm{BMI} \%$, and the significant attentional avoidance of food stimuli among Dutch children, it suggests that merely mean AB scores cannot provide sufficient information. Considering both the attractive and the unhealthy aspect of highcaloric foods (Roefs et al., 2018), the dynamic character of AB for food and for other similar stimuli should be explored.

Both the direct effect of EC on adjusted BMI\% and the moderating effect of EC on the relation between TL-BS variability for food and adjusted BMI\% were not significant in the first study, it is still unclear whether and how EC influences adjusted BMI\%. As there is 
scarcely any study about $\mathrm{AB}$ for food in children from eastern countries, it was less clear whether the positive association between the variability of $\mathrm{AB}$ for food and adjusted BMI\% exists in the children from a totally different culture. Next, we conducted a similar study in China to examine the dynamic natures of $\mathrm{AB}$ for food in Chinese children, and further test the direct effect of EC on adjusted BMI\% and the moderating effects of EC on the relations between the central tendency of $\mathrm{AB}$ for food/the variability of $\mathrm{AB}$ for food and adjusted BMI\%.

\section{Study 2}

The food dot-probe task and the child-version of the attention network task (ANT) were administered among Chinese children in Study 2.

\section{Methods}

\section{Participants}

102 Chinese children across the weight spectrum were recruited from a local primary school in the southwest part of China (Kunming city, Yunnan province). Two children were excluded from further analysis because of missing data and another child was excluded because of poor performance on both tasks (> 25\% mistakes). The final sample consisted of 99 children $\left(97.06 \%\right.$ of the original sample; $M_{\text {age }}=10.98$ years old, $S D_{\text {age }}=0.94$, Range $e_{\text {age }}=$ 9-13 years old).

\section{Materials}

Hunger Questionnaire. A Chinese version of the questionnaire that was used in Study 1.

Food Dot-probe Task. The task was the same as Study 1, except for the food and animal stimuli. We adapted the stimuli according to Chinese children's preferences. 45 children from the same school, not participating in the actual study, rated a pool of 27 food pictures and 20 animal pictures on a 5-point Likert scale, with 1 reflecting not attractive at all, and 5 reflecting very attractive. We selected the first twelve most attractive food and animal pictures as our stimuli $\left(M_{\text {animals }}=3.89, S D_{\text {aniamls }}=0.32 ; M_{\text {food }}=4.14, S D_{\text {food }}=0.28\right)$. 
Child-version Attention Network Task (ANT). A Chinese version of the ANT was used in experiment 1 .

\section{Procedure}

Children were tested in groups (approximately 30 children per group) in their school computer room. The procedure was the same as in Study 1, except that the information on weight and height was collected from the school's annual physical test (this was conducted 1 month before our testing).

\section{Data Reduction and Analysis}

Data Reduction. The inclusion criteria for trials of the food dot-probe task and ANT were the same as in Study 1. Based on these criteria, 2.77\% of trials from the food dot-probe task and $6.02 \%$ of trials from the ANT were discarded. One outlier (3.04 SD above the mean value) in the TL-BS variability for food, one outlier (3.49 SD above the mean value) in the TL-BS variability for animal, and one outlier (3.19 SD above the mean value) in the TL-BS variability for neutral trials were replaced by the respective nearest scores of the whole sample (see Wilcox, 2001) ${ }^{1}$.

Computation of Attentional Indexes. The calculations of all indexes were the same as in Study 1. The mean number of IT-CT pairs was $53.10(S D=4.52)$ for the food condition, $54.12(S D=3.85)$ for the animal condition, and $54.72(S D=4.23)$ for the neutral condition; the mean distance between each IT and CT was 2.58 trials $(S D=0.17)$ for the food condition, $2.61(S D=0.20)$ for the animal condition, and $2.58(S D=0.19)$ for the neutral condition.

Analysis Plan. The analysis plan was the same as in Study 1.

\section{Results}

Demographic Information and Average Performance on Tasks

Participant characteristics and average performance in Study 2 can be seen in Table 1.

\section{Correlations}


The results of zero-order correlations revealed that merely mean attentional bias (AB) scores for animal and executive control (EC) ability were significantly correlated with adjusted $\mathrm{BMI} \%$. Because EC was also significantly related to the mean AB scores for animal, it could be a potential confounder influencing the relation between mean $\mathrm{AB}$ scores for animal and adjusted BMI\%. Therefore, a partial correlation was computed controlling for EC. After controlling for EC, the significant association between adjusted BMI\% and mean AB scores for animal disappeared, $r_{\text {partial }}(96)=-.15, p=.15$. TL-BS variability for food was significantly negatively related to the level of hunger, meaning that children, who were feeling hungrier, showed less variability in attention for food. There were no significant associations between age and the variabilities of attentional bias (TL-BS variabilities). As no mean $\mathrm{AB}$ scores or TL-BS variabilities were related to adjusted $\mathrm{BMI} \%$, there was no need to proceed with the regression and moderation analysis. See Table 4 for an overview of all correlations. 


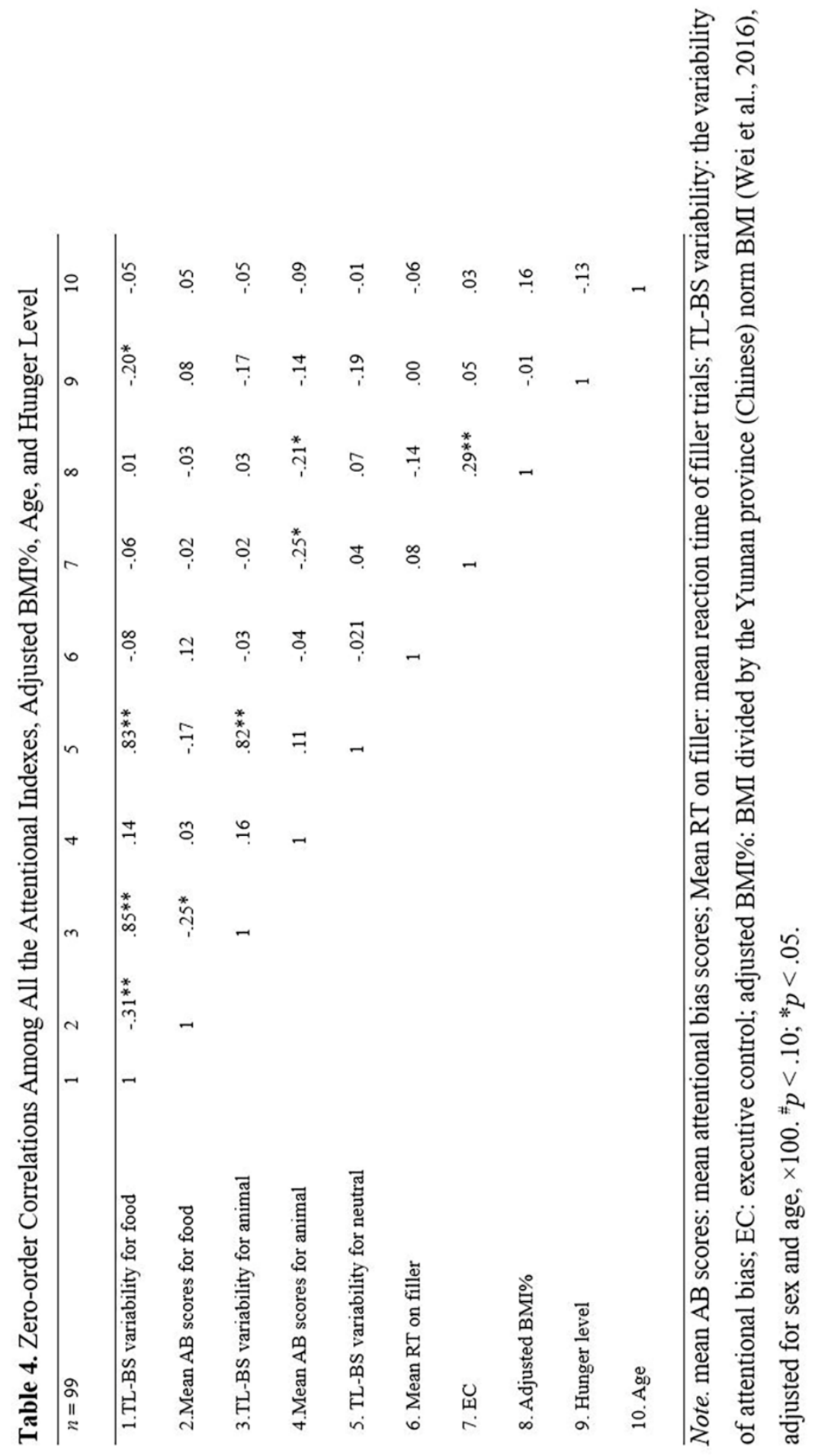


Mean $A B$ for food in the Sample

The result indicated that Chinese children showed marginally significant $(t(105)=1.71, p$ $<$.10.) attentional avoidance of food stimuli.

Comparing the Samples of the Two Countries (Study 1 and 2)

We also tested the differences between the Dutch and Chinese sample. As shown in Table 1, the groups differed on age and level of hunger. Then, we controlled for age and hunger and compared the two samples on TL-BS variability for food/animal/neutral, mean AB scores for food/animal, mean RT on neutral trials and EC ability. The results revealed that except mean AB scores for food and animal, the two samples differed on all other indexes. Compared with Dutch children, Chinese children showed less variability for food/animal/neutral stimuli, responded faster on neutral trials in the food dot-probe task, and showed better EC ability in the ANT.

\section{Discussion}

Unexpectedly, the results of Study 2 demonstrated that among Chinese children, the variability of attentional bias (AB) for food (TL-BS variability for food) was not significantly related to adjusted $\mathrm{BMI} \%$. Execute control (EC) and mean $\mathrm{AB}$ scores for animal were positively related to adjusted $\mathrm{BMI} \%$, but the association between mean $\mathrm{AB}$ scores for animal and adjusted BMI\% disappeared after controlling for EC. The children only showed more variability in attention for food, when they were feeling less hungry. Instead of paying attention to food stimuli, Chinese children showed marginally significant attentional avoidance of food stimuli. Furthermore, there was a big group difference (Dutch children vs Chinese children; partial $\eta^{2}>0.20$ ) in performances (TL-BS variabilities, mean RT on neutral, and EC) after controlling for age and hunger.

Unlike in Dutch children, the positive association between the variability of AB for food and adjusted BMI\% was not significant in Chinese children. Interestingly, in Study 2, EC ability was directly negatively related to adjusted BMI\%, whereas this correlation was not significant in Study 1. Moreover, Chinese children showed extremely high EC ability compared with Dutch children. So, it might be that the difference in EC ability between the countries is a reason for the divergence in AB findings. TL-BS variability for food may only 
be related to adjusted BMI\% in children with weaker executive control ability (like the Dutch children in our Study 1), whereas, other vulnerability factors might play an important role in adjusted BMI\% among children with stronger executive control ability (like the Chinese children in our Study 2). Taken together, results suggest that EC is an important variable explaining differences in BMI, which is also consistent with previous studies (e.g., Lavagnino, Arnone, Cao, Soares, \& Selvaraj, 2016). On its turn, cultural differences may contribute to differences in EC ability.

\section{General Discussion}

The current studies investigated both the mean attentional bias (AB) for food and the dynamic nature of this $\mathrm{AB}$, among Dutch and Chinese children by using the food dot-probe task. In addition, we explored the association between EC ability and adjusted BMI\%. Finally, we tested the moderating role of EC ability in the relationship between $\mathrm{AB}$ (variability) and BMI.

Our results showed that (1) TL-BS variability for food was positively related to adjusted BMI\% in Dutch children; (2) after controlling for mean AB scores for food, TL-BS variability for food still significantly predicted the variance in adjusted BMI\% among Dutch children; (3) EC ability was not related to adjusted BMI\% in Dutch children and it did not significantly moderate the relation between TL-BS variability for food and adjusted BMI\% in both Dutch and Chinese children, whereas EC ability was negatively related to adjusted $\mathrm{BMI} \%$ in Chinese children.

Consistent with our first hypothesis, in Dutch children specifically TL-BS variability for food predicted variance in adjusted BMI\%, not TL-BS variability for animal or mean $\mathrm{AB}$ scores for food. This is in line with the results of a recent study from our lab, in which TL-BS variability for food explained additional variance in adjusted BMI\% after controlling for mean AB scores for food (Liu et al., 2019). Kruijt and colleagues (2016) have criticized the TL-BS methodology and found that TL-BS variability was sensitive to the increasing standard deviation of reaction time (RT) in the absence of a bias, which means a higher $S D$ may reflect error variance and the TL-BS variability cannot differentiate between measurement error and bias variability. If the TL-BS variability for food just reflects the variance in general responding, then we would get the same results of the TL-BS variability for cute animals. However, our current study revealed that merely the TL-BS variability for 
food, not for cute animals, could significantly predict the variance in BMI. So, it seems that at least the TL-BS variability for food can capture some features of bias variability for food stimuli.

The current study showed that there was a marginally negative association between mean $\mathrm{AB}$ scores for food and adjusted BMI\% in Dutch children. In addition, Dutch children expressed significant and Chinese children expressed marginally significant attention avoidance of food stimuli. So, both groups focused more on neutral than on food stimuli, and Dutch children with a higher BMI showed even more avoidance of food stimuli. Note that these findings are not in line with theory (Field \& Cox, 2008), but do not stand on their own, attentional avoidance of food stimuli has been observed in prior research (e.g., Nummenmaa et al., 2011; Gearhardt, Treat, Hollingworth, \& Corbin, 2012). Overall, our results together with inconsistent results produced in the field of $\mathrm{AB}$ for critical stimuli (e.g., Bantin, Stevens, Gerlach, \& Hermann, 2016; Field et al., 2016; Roefs et al., 2015), like food and emotionrelated stimuli, all demonstrate the importance of developing other effective computing methods to provide additional information about $\mathrm{AB}$ for target stimuli, and the importance of focussing on the dynamic nature of $\mathrm{AB}$ for target stimuli. As high-caloric food can be associated with both eating enjoyment and weight gain concern, our attention may switch rapidly between approach and avoidance for food (Roefs et al., 2018), which is better captured by TL-BS.

Unexpectedly, we did not find a similar result among Chinese children, and for them, there was no significant association between TL-BS variability for food and adjusted BMI\%. The variability in AB for food may have been limited due to Chinese children's strong EC ability, and possibly, therefore, no significant association between the TL-BS variability for food and adjusted BMI\% was found. In addition, different from our hypotheses, the overall moderating effect of EC was not significant in both Dutch and Chinese children. EC ability was only negatively related to adjusted BMI\% among Chinese children. Considering the significantly different EC abilities between Chinese and Dutch children, it seems that there might be a floor effect in the Dutch sample, with generally low EC ability scores, and therefore not enough variability to observe significant moderation of the relationship between attentional variability for food and BMI. Similarly, there might be a ceiling effect in the Chinese sample, with generally high EC ability, and therefore not enough variability to 
observe significant moderation. It is partly consistent with previous research that $\mathrm{AB}$ for alcohol was related to alcohol use merely in participants with low EC ability (van HemelRuiter et al., 2015). However, it is also possible that increased EC directly leads to a lower adjusted BMI\% (like the result in Study 2), or alternatively, EC may be indirectly related to a lower adjusted BMI\% mediated the TL-BS variability for food. Future studies, with sufficient variability in EC ability among participants, are required to test whether EC moderates the relation between TL-BS variability for food and BMI, or EC is directly related to BMI, or EC is indirectly related to BMI, mediated via TL-BS variability for food.

Apart from our main purposes, Study 1 also revealed that the level of hunger was positively related to TL-BS variability for food, animal, and neutral pictures among Dutch children. It might be the case that hunger caused difficulties to maintain concentration, and combined with weaker EC ability, could be an explanation for these results. In contrast, Chinese children showed a negative association between hunger level and TL-BS variability for food. It may be caused by strong EC ability as well. As the mean hunger level of Chinese children was moderate $($ Mean $=4.36)$, they might use the strong EC ability to force themselves to concentrate on the task and not on the unreachable food stimuli.

There are some potential reasons for the difference in EC ability between Dutch and Chinese children. First, Chinese schools emphasize self-control, and children are required to strictly comply with school discipline, which can be beneficial for developing EC ability. Second, intensive training on abilities such as following directions and concentrating on the targets in Chinese schools (Lan et al., 2009) can also contribute to EC ability. Finally, the Chinese children were tested collectively within their class in the computer room of their school with their teachers as assistants, while Dutch children were tested in several random groups at one of the classrooms or computer room without their teachers. The testing scene of Chinese children is more similar to a class situation, and it might enable students to concentrate better on their task.

The current findings also shed some light on the usefulness of some types of overweight/ obesity prevention or intervention programs. Attention bias modification (ABM) training (MacLeod, Rutherford, Campbell, Ebsworthy, \& Holker, 2002), a modified version of the dot-probe task, is used to change individuals' AB for food. It assumes that increased 
$\mathrm{BMI}$ and unhealthy dietary habits are related to $\mathrm{AB}$ for high-calorie foods, and $\mathrm{AB}$ for highcalorie foods can be changed via manipulating and measuring the reaction time-based $\mathrm{AB}$ (Kakoschke, Kemps, \& Tiggemann, 2014; Werthmann, Field, Roefs, Nederkoorn, \& Jansen, 2014). However, the current study found a marginally negative association between mean $\mathrm{AB}$ for high-calorie foods and adjusted $\mathrm{BMI} \%$, which adds doubt on the applicability of $\mathrm{ABM}$ for food, since it is meaningless to train individuals who do not show $\mathrm{AB}$ for highcalorie foods to avoid high-calorie foods. Apart from that, the different results between Chinese and Dutch children also imply to us that it is necessary to consider the characteristic of different groups when design or choose corresponding prevention and intervention programs.

There are several limitations in our current study. First, the food dot-probe task only provided RT-based AB measures, and other more direct information about $\mathrm{AB}$ (like eye movement data) should be included in future research. Second, we only measured general EC but not food specific, which is more strongly related to eating behaviors (Houben, Nederkoorn, \& Jansen, 2014; Svaldi, Naumann, Trentowska, \& Schmitz, 2014). Future studies should further examine the influence of food-specific EC on BMI and TL-BS variability.

In summary, the current study explored the relationships among TL-BS variability for food/animal, mean AB score for food/animal, EC and adjusted BMI\% and produced informative results. The results showed that the predictive effect of TL-BS variability for food on adjusted BMI\% only existed among Dutch children. For Chinese children, EC ability was directly related to adjusted $\mathrm{BMI} \%$. Therefore, future AB-related studies should further investigate the relationship between the variability of $\mathrm{AB}$ for food and adjusted $\mathrm{BMI} \%$ in participants with a wide range of EC ability and concentrate on the effects of both the variability of $\mathrm{AB}$ for food and $\mathrm{EC}$ ability on weight control. Besides, since there are lots of factors may contribute to overweight and obesity, different population features should be considered when design food-related studies, especially clinical prevention and intervention. 
Chapter 5

\section{Fluctuations in Attentional Bias for Food and the Role of Executive Control}

Submitted as:

Liu, Y., Roefs, A., \& Nederkoorn, C. (2021). Fluctuations in attentional bias for food and the role of executive control. Appetite. 


\begin{abstract}
Research has shown repeatedly that overweight and obesity are associated with more fluctuations in attentional bias (AB) for food. More specifically, people with a high BMI more frequently alternate their attention towards and away from food over time. The current study proposed and tested a model on the mechanism behind the positive association between variability of $\mathrm{AB}$ for food and BMI. It was hypothesized that poor executive control (response inhibition and sustained attention) would be related to both weight gain and fluctuations in $\mathrm{AB}$ for food and this would, at least partly, explain the significant relationship between variability of $\mathrm{AB}$ for food and BMI. To test our hypotheses, 99 female participants completed both an online food dot-probe task and an online stop-signal task. It was found that response inhibition was significantly related to BMI, whereas, contrary to our hypotheses, neither executive control was significantly related to $\mathrm{AB}$ for food, nor variability of $\mathrm{AB}$ for food was significantly related to BMI. In conclusion, this study revealed a relation between poor executive control and overweight/obesity. However, the role of executive control in fluctuations in attention for food could not be established.
\end{abstract}

Keywords: executive control, inhibitory control, sustained attention, attentional bias variability, BMI 


\section{Introduction}

The incentive salience model suggests that a rewarding stimulus, like a palatable food, is salient and draws attention. Such incentive salience of palatable food is proposed to be a characteristic of people with overweight or obesity (Berridge, 2009; Berridge, Ho, Richard, \& DiFeliceantonio, 2010). Accordingly, it is generally believed that people with a high body mass index (BMI) are characterized by a stable attentional bias (AB) for high-calorie palatable foods, and some studies support this view (e.g., Castellanos et al., 2009; Hendrikse et al., 2015; Nijs \& Franken, 2012). However, empirical evidence does not consistently support this view. That is, studies have not only found evidence for increased $A B$ for food in obesity (e.g., Hume, Howells, Rauch, Kroff, \& Lambert, 2015), but also for decreased AB for food (e.g., Fang et al., 2019), or no difference in AB for food (e.g., Doolan, Breslin, Hanna, Murphy, \& Gallagher, 2014), all as compared to healthy-weight control participants.

More recently, several studies revealed that people with a high BMI were characterized by more frequent fluctuations in $\mathrm{AB}$ for food than healthy-weight people, with $\mathrm{AB}$ frequently alternating between attention towards versus away from food. This finding has been observed in both adult and Dutch child participants, but not in Chinese children (Liu, Nederkoorn, Roefs, 2019; Liu, Roefs, Werthmann, Nederkoorn, 2019; Liu, Nederkoorn, Roefs, 2021). More specifically, these studies computed trial-level bias scores (TL-BS) (Zvielli, Bernstein, \& Koster, 2015) to quantify participants' AB for food stimuli in a food dot-probe task on a trial level, instead of averaged across the entire task.

The computation of TL-BS is based on the assumption that an AB for critical stimuli is not stable, but fluctuates between approach and avoidance over time, over the course of the task. TL-BS variability is typically derived from TL-BS, and reflects the extent of fluctuations in $\mathrm{AB}$ for critical stimuli. TL-BS variability has demonstrated higher split-half reliability and shown to be a significantly better predictor of several indicators of psychopathology and of an increased BMI as compared to the widely used mean AB score (e.g., Amir, Zvielli, \& Bernstein, 2016; Caudek, Ceccarini, \& Sica, 2017; Liu, Nederkoorn, et al., 2019; Liu, Roefs, et al., 2019; Zvielli, Vrijsen, Koster, \& Bernstein, 2016; Zvielli et al., 2015). However, the underlying mechanism behind the positive association between TL- 
BS variability for food and BMI is still unclear, and the current study aims to uncover this mechanism.

It has been suggested that attention allocation depends on both the salient nature of stimuli (bottom-up process) and the momentary motivations of observers (top-down process) (Katsuki \& Constantinidis, 2014). Therefore, a previous study (Liu et al., 2021) tested the hypothesis that fluctuations in the activation of food-related goals might underlie fluctuations in $\mathrm{AB}$ for food: people might vary between wanting to approach and consume food, if their hedonic goal is more active, and wanting to avoid high caloric foods if their health goal is more active. In a state in which one goal is dominant, people would show less variability in $\mathrm{AB}$ for food as compared to a neutral state. However, results showed that the experimental manipulation of food-related goals (health, hedonic, and neutral) did not significantly influence TL-BS variability for food. It should be noted that the experimental manipulation in this study was not that strong and this study did not successfully induce a health motivation. It is unclear whether a stronger manipulation would have the expected effect. In addition, TL-BS variability for food was not related to restrained eating in several studies (Liu, Nederkoorn, et al., 2019; Liu, Roefs, et al., 2019), while dietary restraint reflects the activation of two opposite goals that likely alternate often in restrained eaters (Stroebe, van Koningsbruggen, Papies, \& Aarts, 2013). Taken together, there is no evidence for the idea that fluctuations in $\mathrm{AB}$ for food are caused by fluctuations in activations of these two opposite goals. Therefore, other factors need to be considered, and executive control capacity is such a candidate that might influence variability of $\mathrm{AB}$ for food.

Executive control is a top-down process that is necessary to control attention, thoughts, emotions, or behaviors to override a strong internal predisposition or external lure (Diamond, 2013). Many studies have demonstrated that overweight and obesity are related to weaker executive control (e.g., Mamrot \& Hanć, 2019; Yang, Shields, Guo, \& Liu, 2018). For example, people with overweight/obesity showed more impulsivity on behavioral tasks (e.g., Chen, Jia, \& Woltering, 2018; Nederkoorn, Smulders, Havermans, Roefs, \& Jansen, 2006; Nederkoorn, Coelho, Guerrieri, Houben, \& Jansen, 2012), have decreased grey matter volumes in the prefrontal cortex (e.g., Medic et al., 2016), and showed reduced activation of frontal inhibitory regions and significantly more false positive responses during a foodspecific go/no-go task (Batterink, Yokum, \& Stice, 2010). This reduced executive control 
may make people more vulnerable to tempting food, as they might be less able to inhibit their appetitive responses and keep their health goals active (e.g., Jensen, Duraccio, Carbine, Barnett, \& Kirwan, 2017; Riggs, Spruijt-Metz, Sakuma, Chou, \& Pentz, 2010; Stevenson \& Francis, 2017). This can therefore lead to more food intake and subsequent weight gain.

In addition, lower executive control may also make people less able to have a consistent, prolonged attention to critical stimuli, therefore, causing more variability in $\mathrm{AB}$ for such critical stimuli. This assumption was supported by results from several empirical studies. Specifically, it was found that attentional control ability was significantly and negatively related to both TL-BS variability for negative words and anxiety (Clarke et al., 2020). In addition, the association between variability of AB for food and BMI was no longer significant for people high in attentional control (Liu, Nederkoorn, et al., 2019). So, it is reasonable to expect that executive control could, at least partly, explain the positive relationship between variability of $\mathrm{AB}$ for food and $\mathrm{BMI}$.

Therefore, we propose and test a model on a potential mechanism behind the relationship between increased variability of $\mathrm{AB}$ for food and BMI. More specifically, low executive control could be related to increased food intake and body weight, and it could also be related to more variability in $\mathrm{AB}$ for food. This would then at least partly, explain the association between variability of AB for food and BMI (see Figure 1). It was hypothesized that (1) executive control would be significantly related to BMI; (2) executive control would be significantly related to variability of $\mathrm{AB}$ for food; (3) variability of $\mathrm{AB}$ specifically for food stimuli would be significantly and positively related to BMI; (4) the relation between variability of $\mathrm{AB}$ for food and BMI would be weaker after controlling for executive control. 
Figure 1. Hypothesized Model to Explain the Underlying Mechanism of the Positive Correlation Between TL-BS Variability for Food and BMI.

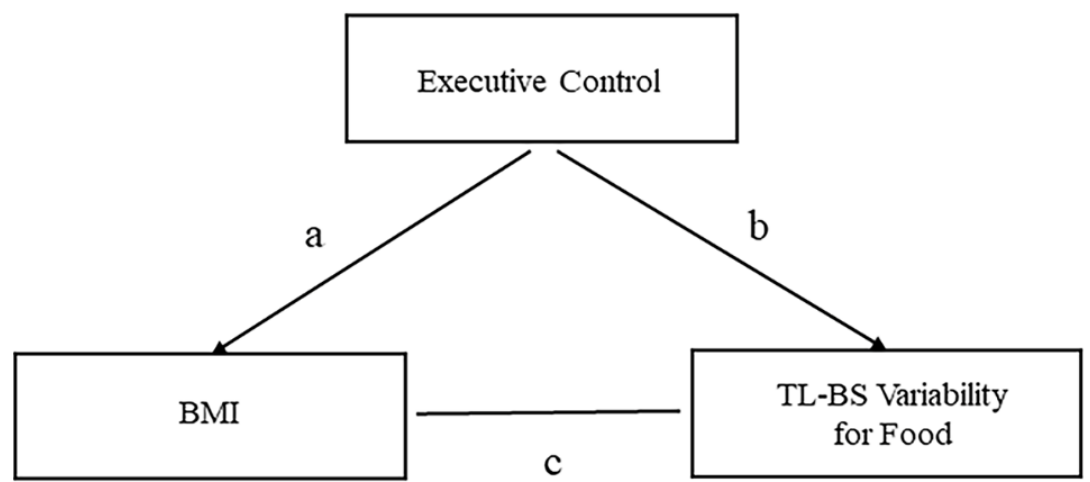

Note. Executive control was represented by response inhibition and sustained attention in a stop-signal task.

\section{Method}

\section{Participants}

The participants were recruited via an online poster in a participants recruitment system. To cover a wide range of BMI and to eliminate potential sex and age effects on task performance (Hallam, Boswell, DeVito, \& Kober, 2016), Dutch females aged between 18 to 60 were selected for the current study according to their self-reported body mass index (BMI; $\left.\mathrm{kg} / \mathrm{m}^{2}\right)$. The sample size was determined by a power analysis via $\mathrm{G}^{*}$ Power. For a regression model (fixed model, $\mathrm{R}^{2}$ increased, medium effect size, $\alpha=.05$, power $=.90$ ) with a maximum of 6 predictors, it was suggested to include at least 99 participants. Finally, 114 participants completed the whole experiment, of which 15 participants were excluded for further analysis because of poor task performance, meaning 99 participants remained in the final analyses. The current study was approved by the Ethical Committee of the Faculty of Psychology and Neuroscience, Maastricht University. All participants agreed on the informed consent, in which they were informed about the procedure, storage of data, and their right to withdraw from participation without any consequence. The study protocol was pre-registered at AsPredicted (https://aspredicted.org/ge7tq.pdf ). 


\section{Materials}

All questionnaires were administered online via Qualtrics and all the tasks were conducted online via Inquisit Web version 6.3.5 (Millisecond Software, Seattle, WA, USA).

\section{Questionnaires}

Dietary Restraint. Participant's intention to restrict food intake was assessed by the Restraint Scale (RS; 11-item self-report scale; Herman \& Polivy, 1980) and Cronbach's $\alpha$ was 0.77 in the current study.

Hunger Level. Participant's hunger level was measured on a $100-\mathrm{mm}$ visual analogue scale (VAS) by asking "How hungry are you right now?", with 0 indicating no hunger at all and 100 indicating extreme hunger.

Food Liking. Food liking of the high-calorie food stimuli that were presented in the fooddot probe task was measured on a 100-mm VAS, with 0 indicating a lack of liking and 100 indicating extreme liking.

\section{Food Dot-probe Task}

Trial procedure. Attentional bias $(\mathrm{AB})$ was measured by the food dot-probe task (MacLeod et al., 1986). First, a central fixation cross was presented for $500 \mathrm{~ms}$, followed by two images that were simultaneously presented side-by-side for $500 \mathrm{~ms}$. Then, one of the images was replaced by a probe $(*)$, which randomly and equally often was presented on the left or right side of the screen. Participants were required to focus on the central fixation cross and respond to the probe as quickly and accurately as possible. The probe was presented until a response or for a maximum of $2000 \mathrm{~ms}$.

Stimuli in the food dot-probe task. Two categories of neutral stimuli: familiar household items and musical instruments, were used in the current study. Musical instrument pictures were included to test whether variability of $\mathrm{AB}$ specifically for food stimuli but not for neutral nonfood stimuli is related to BMI, so, the same as palatable food pictures, they were relevant stimuli. The palatable food stimuli and musical instrument stimuli in the current study were adapted from Werthmann et al. (2011) and Werthmann et al. (2015). All 
image pairs were subjectively matched as closely as possible with regard to the shape, color, brightness, and size of the depicted object.

Trial Types. The current task included five different types of trials: food incongruent trials (ITs), food-congruent trials (CTs), musical instrument ITs, musical instrument CTs, and filler trials (NTs). On food trials, a food-household item picture pair was presented, whereas on musical instrument trials, a musical instrument-household item picture pair was presented. On ITs, the probe appeared in the location of the household item stimulus, whereas on CTs, the probe appeared in the location of the target stimulus (food or musical instrument). NTs included two household item stimuli to reduce possible habituation to stimuli that might occur if all trials contained relevant stimuli.

Number of Trials. Equal numbers of food CTs, musical instrument CTs, food ITs, musical instrument ITs, and NTs (200 trials in total) were evenly and randomly distributed across two blocks. Food trials (CTs/ITs) consisted of 20 pairs of palatable food (target) and household item stimuli; musical instrument trials (CTs/ITs) also consisted of 20 pairs of musical instrument (target) and household item stimuli; filler trials consisted of 10 paired household item stimuli. Different household item pictures were used for food trials, musical instrument trials, and filler trials. Each of the stimulus pairs was presented 4 times and each picture appeared equally often on the left and on the right side of the screen.

\section{Stop-signal Task}

The stop-signal task was conducted according to a guideline proposed by Verbruggen and colleagues (2019). The task included both a go task and a stop task, presented counterbalanced in each block. For the go task participants were required to respond to an arrow as fast as possible, while for the stop task, participants were required to withdraw any response if they heard a tone. A dynamic tracking procedure was used to adjust the delay between the presentation of an arrow and the stop sound signal in the stop task (stop-signal delay: SSD).

Trial procedure. Participants first performed 34 practice trials, which were followed by the actual task consisting of three blocks with 72 trials in each block. Each trial started with a central fixation circle $(500 \mathrm{~ms})$, and then an arrow was presented within this 
circle that either pointed right or left. Participants were required to press "F" key on the keyboard if the arrow pointed left and " $\mathrm{J}$ ' key on the keyboard if the arrow pointed right (go task). In $25 \%$ of the trials, a beep sound was presented (stop-signal) to indicate the inhibition of the response (stop task). SSD (starting at $250 \mathrm{~ms}$ ) was adjusted up or down in steps of 50 ms depending on the participant's performance. SSD increased if the previous signal stop was successful (up to $1150 \mathrm{~ms}$ ), whereas it decreased if the previous signal stop was unsuccessful (down to $50 \mathrm{~ms}$ ).

\section{Procedure}

The experiment was conducted fully online. First, a Qualtrics link to the pre-task survey was sent to participants who had signed up for the study via the online participant recruitment system. The survey started with an informed consent and next some screening questions were asked to check the inclusion criteria (age:18-60 years old, sex: female, and vision: normal or corrected-to-normal). If the participant did not meet the inclusion criteria, the study participation was ended. If the participant met all inclusion criteria, the level of hunger was measured, intermixed with several filler questions (e.g., how many cups of water did you drink yesterday?). Next, the participant was automatically directed to the food dot-probe task and then to the stop-signal task. The participant could take a short break between blocks and between tasks, and it was suggested to limit each break to 1 minute. Next, liking of the food pictures, the restraint scale, and self-reported height and weight were assessed in another Qualtrics online survey. Finally, a debriefing was provided. The whole procedure lasted about 1 hour. Afterwards, the participant was thanked for her participation and received a small reward (10-euro gift voucher).

\section{Data Reduction and Analysis}

AB Scores

Mean AB for food/musical instrument was calculated by subtracting the mean RTs of the food/musical instrument CTs from the mean RTs of the food/musical instrument ITs. So, a positive value reflects an $\mathrm{AB}$ towards food/musical instrument, and a negative value reflects an $\mathrm{AB}$ away from food/musical instrument. To obtain sequential TL-BSs, first, each food/musical instrument CT was paired with a food/musical instrument IT that was as close 
as possible in time and no further than five trials away. Next, the food/musical instrument CT in each pair was subtracted from the food/musical instrument IT in that pair. TL-BS variability for food/musical instrument was computed using the sum of absolute distances between sequential TL-BSs divided by the total number of TL-BSs (Zvielli et al., 2015). The mean numbers of IT-CT pairs in the food trials and musical instrument trials were 57.14 and 57.18 respectively. The mean distances between each IT and CT were 1.44 and 1.46 trials per trial condition respectively.

\section{Executive Control}

Because the mean stop-signal reaction time (mSSRT) is adopted most frequently in the food domain, and to make our results comparable to others, we adopted mSSRT to estimate the time required to stop the initiated go-process (response inhibition). A longer mSSRT reflects poorer inhibition ability. According to Verbruggen, Logan, and Stevens (2008), the first trial in each block was regarded as practice and was not included in analyses. The mSSRT was computed by subtracting the mean SSD on the stop task from the mean reaction time (RT) on the go task.

Sustained attention. The standard deviation of RTs ( $S D$ of RT) on the go task was used as an indicator of sustained attention (Hultsch, MacDonald, \& Dixon, 2002) A higher score is taken to reflect poorer sustained attention (Bellgrove, Hester, \& Garavan, 2004).

\section{Data Reduction}

Participants were excluded from further analyses: (1) if they made mistakes in more than 25\% of the trials in the food dot-probe task; (2) if they successfully inhibited less than $25 \%$ or more than $75 \%$ of the trials in the stop-signal task; (3) if their mean RT on unsuccessful stop trials was numerically longer than their mean RT on go trials in the stop-signal task. 15 participants were excluded because of poor performance on the food dot-probe task, eight because of poor performance on the stop-signal task and two because of poor performance on both tasks.

RTs in the food dot-probe task were excluded from further analyses if they were error responses, faster than $200 \mathrm{~ms}$, slower than $1500 \mathrm{~ms}$, or deviate more than $3 \mathrm{SDs}$ from each participant's mean RT after excluding error responses, faster than $200 \mathrm{~ms}$ trials, and 
slower than $1500 \mathrm{~ms}$ trials. Accordingly, 3.43\% of trials from the food dot-probe task were discarded.

Finally, for all dependent and independent variables, outliers $(M \pm 3 S D)$ were replaced by the corresponding nearest score of the whole sample (see Wilcox, 2001) ${ }^{6}$. One outlier (3.17 SD above the mean value) on BMI, one outlier (4.10 SD above the mean value) on mean RT of food, three outliers (3.56 SD, 3.36 SD, and 3.05 SD above the mean value) on TL-BS variability for food, and one outlier (4.11 SD above the mean value) on TL-BS variability for musical instrument were replaced by the nearest value of the whole sample.

\section{Analysis Plan}

First, correlations were computed to investigate if potential confounders (age, hunger level, and food liking) were related to dependent variables (BMI and AB scores). Potential confounders were included in further analyses if they were related to dependent variables (Gruijters, 2016). Next, correlations between BMI and TL-BS variability for musical instrument/mean $\mathrm{AB}$ for food were computed, to test if possible effects would be specific for variability of $\mathrm{AB}$ for food, or would be more general for $\mathrm{AB}$ variability/mean $\mathrm{AB}$. Next, two hierarchical regressions were conducted to test our hypotheses: Model 1 tested the relation between executive control (mSSRT and $S D$ of RT) and TL-BS variability for food (step 1; path b Figure 1). BMI was added in step 2 to test if it could explain additional variance in TL-BS variability (path c Figure 1). Model 2 tested the relation between executive control and BMI (step 1; path a Figure 1). TL-BS variability was added in step 2 to test if it could explain additional variance in BMI (path c Figure 1). Multicollinearity was checked for each regression model, and all variance inflation factors (VIF) were smaller than 5 (Kutner, Nachtsheim, \& Neter, 2005).

\section{Results}

\section{Demographic Information and Average Performance on Tasks}

\footnotetext{
${ }^{6}$ The analyses with unchanged values or exclusion of these values showed the same pattern of results and led to the same conclusions.
} 
Participants' demographic information and average performance on the tasks are displayed in Table 1.

Table 1. Participant Characteristics and Average Performances on Tasks

\begin{tabular}{lccc}
\hline variable & $M$ & $S D$ & Range \\
\hline Age (year) & 38.10 & 12.17 & $19.00-59.00$ \\
BMI & 27.07 & 5.43 & $17.90-41.52$ \\
RS score & 13.67 & 5.03 & $5-29$ \\
Hunger level & 21.16 & 23.77 & $0-84$ \\
Food liking & 62.52 & 15.33 & $14.45-90.85$ \\
TL-BS variability for food & 83.69 & 29.85 & $37.68-168.79$ \\
TL-BS variability for musical instrument & 83.57 & 30.22 & $37.29-162.31$ \\
Mean AB for food & -2.41 & 16.39 & $-38.55-47.27$ \\
Mean AB for musical instrument & 6.99 & 14.81 & $-28.20-43.26$ \\
mSSRT & 223.24 & 50.88 & $97.30-372.49$ \\
SD of RT go-trials stop-signal task & 139.68 & 49.90 & $50.60-261.41$
\end{tabular}

Note. BMI: body mass index; RS: restraint scale (Herman \& Polivy, 1980); hunger level: hunger level of participants (0-100 mm VAS); food liking: average food liking score for the food stimuli presented in the food-dot probe task (0-100 mm VAS). TL-BS: trial-level bias scores; AB: attentional bias; mSSRT: mean method of stop-signal reaction time; SD of RT: standard deviation of reaction times.

\section{Correlations}

\section{Potential Confounders}

The results of zero-order correlations showed that only age was significantly and positively related to TL-BS variability for food. There were no significant correlations between other potential confounders and any other dependent variables, $r s=-.18$ to .15 , all $p s>.07$. Therefore, age was controlled for when the dependent variable was TL-BS variability for food. More details can be found in Table 2.

\section{Control Analyses}

Correlations were computed to test if effects on BMI were specific for TL-BS variability for food, or more general, for TL-BS variability for musical instrument and mean AB for food 
as well. The results of zero-order correlations revealed that neither TL-BS variability for musical instrument, nor mean AB for food, was significantly correlated with BMI. See Table 2 for more details.

Table 2. Correlations Among BMI, TL-BS variability, mean AB for food, and Potential Confounders

\begin{tabular}{lccccccc}
\hline$n=99$ & 1 & 2 & 3 & 4 & 5 & 6 & 7 \\
\hline 1.BMI & 1 & .09 & .08 & -.06 & .10 & .09 & .15 \\
2.TL-BS variability for food & & 1 & $.86^{* *}$ & .04 & $.30^{* *}$ & .05 & -.18 \\
3.TL-BS variability for musical instrument & & & 1 & .12 & $.29^{* *}$ & .11 & -.06 \\
4.Mean AB for food & & & & 1 & .003 & .09 & .01 \\
5. Age & & & & 1 & .02 & $-.21^{*}$ \\
6. Hunger level & & & & & 1 & .16 \\
7. Food liking & & & & & & 1 \\
\hline
\end{tabular}

Note. BMI: body mass index; TL-BS: trial-level bias scores; AB: attentional bias; RS: restraint scale (Herman \& Polivy, 1980); hunger level: hunger level of participants (0-100 mm VAS); food liking: average food liking score for the food stimuli presented in the fooddot probe task $(0-100 \mathrm{~mm}$ VAS $) ;{ }^{*} p<.05, * * p<.01$.

\section{Regression Analyses}

Hierarchical Regression Analysis for Executive Control and BMI Predicting TL-BS Variability for Food

The control variable age significantly explained variance in TL-BS variability for food, $F(1$, $97)=9.25, R^{2}$ change $=0.09, p<.01$. However, contrary to hypothesis, after controlling for age, neither mSSRT and $S D$ of RT (path b Figure 1), entered in the first step, $F(2,95)=1.45$, $R^{2}$ change $=0.03, p=.24$, nor BMI (path c Figure 1), entered in the second step, $F(1,94)=$ $0.31, R^{2}$ change $=0.003, p=.58$, significantly increased the explained variance in TL-BS variability for food. See Table 3.

Table 3. Hierarchical Regression Analysis for Executive Control and BMI Predicting TL-BS Variability for Food

\begin{tabular}{lccccc}
\hline variables & $B$ & $S E B$ & $\beta$ & $t$ & $95 \%$ CI \\
\hline Age & 0.72 & 0.24 & 0.30 & $3.04 * *$ & {$[0.25-1.20]$} \\
\hline
\end{tabular}




\begin{tabular}{lccccc}
\hline Step 1 & & & & & \\
Age & 0.70 & 0.24 & 0.28 & $2.93^{* *}$ & {$[0.22-1.17]$} \\
mSSRT & 0.03 & 0.06 & 0.06 & 0.58 & {$[-0.08-0.15]$} \\
SD of RT in the stop-signal task & 0.09 & 0.06 & 0.16 & 1.62 & {$[-0.02-0.21]$} \\
Step 2 & & & & & \\
Age & 0.68 & 0.24 & 0.28 & $2.84^{* *}$ & {$[0.21-1.16]$} \\
mSSRT & 0.02 & 0.06 & 0.04 & 0.41 & {$[-0.09-0.14]$} \\
SD of RT in the stop-signal task & 0.10 & 0.06 & 0.16 & 1.63 & {$[-0.02-0.21]$} \\
BMI & 0.31 & 0.56 & 0.06 & 0.56 & {$[-0.80-1.41]$} \\
\hline
\end{tabular}

Note. mSSRT: mean stop-signal reaction time; $S D$ of RT: standard deviation of reaction times on go trials; BMI: body mass index; $* * p<.01$.

Hierarchical Regression Analysis for Executive Control and TL-BS Variability for Food Predicting BMI

In line with our hypothesis, mSSRT and SD of RT (path a Figure 1), entered in the first step, significantly increased the explained variance in BMI, $F(2,96)=3.83, R^{2}$ change $=0.07, p$ $<.05$. More specifically, the predictor mSSRT, reflecting poorer response inhibition ability, but not $S D$ of RT, was significantly associated with increased BMI. Inconsistent with hypothesis, entering TL-BS variability for food in the second step did not significantly explain more variance in BMI (path c Figure 1), $F(3,95)=0.69, R^{2}$ change $=0.01, p=.41$. See Table 4.

Table 4. Hierarchical Regression Analysis for Executive Control and TL-BS Variability for Food Predicting BMI

\begin{tabular}{lccccc}
\hline variables & $B$ & $S E B$ & $\beta$ & $t$ & $95 \%$ CI \\
\hline Step 1 & 0.03 & 0.01 & 0.27 & $2.75^{* *}$ & {$[0.01-0.05]$} \\
mSSRT & -0.002 & 0.01 & -0.02 & -0.21 & {$[-0.02-0.02]$} \\
SD of RT in the stop-signal task & & & & & \\
Step 2 & 0.03 & 0.01 & 0.27 & $2.70 * *$ & {$[0.01-0.05]$} \\
mSSRT & -0.004 & 0.01 & -0.04 & -0.35 & {$[-0.03-0.02]$} \\
SD of RT in the stop-signal task & 0.02 & 0.02 & 0.08 & 0.83 & {$[-0.02-0.05]$} \\
TL-BS variability for food & & & & & \\
\end{tabular}


Note. mSSRT: mean stop-signal reaction time; $S D$ of RT: standard deviation of reaction times on go trials; TL-BS: trial-level bias scores; $* * p<.01$.

\section{Discussion}

The current study investigated whether executive control (response inhibition and sustained attention) could partly explain the positive relationship between TL-BS variability for food and BMI, as suggested in previous studies (Clarke et al., 2020). More specifically, we expected that low executive control would be related to both a higher BMI and to larger variability in $\mathrm{AB}$ for food, and thereby partly explaining the relationship between variability in $\mathrm{AB}$ for food and BMI.

Contrary to the hypothesis, we found no evidence that executive control can explain the positive relationship between TL-BS variability for food and BMI. More specifically, consistent with our hypothesis, response inhibition (mean method of stop-signal delay, mSSRT) was significantly related to BMI. However, inconsistent with our hypotheses, sustained attention (standard deviation of reaction times in correct go trials of the stop-signal task, $S D$ of RT) was not significantly related to BMI. Moreover, executive control was not significantly related to variability of $\mathrm{AB}$ for food, and variability of $\mathrm{AB}$ for food was not significantly related to BMI.

The significant relationship between poor response inhibition ability and higher BMI has been observed in several meta-analyses (e.g., Favieri, Forte, \& Casagrande, 2019; Mamrot \& Hanć, 2019; Yang et al., 2018). The current study also found that participants high in BMI showed longer mSSRT, which reflects less efficiency in inhibiting responses. This fits with the idea that lower executive function is associated with excessive high-calorie food intake (e.g., Houben, 2011) and poorer adherence to physical activity (e.g., Riggs, Chou, Spruijt-Metz, \& Pentz, 2010). It should be acknowledged that the causality between executive function and BMI is still unclear, and previous studies suggest this relationship might be bidirectional (e.g., Favieri et al., 2019). So, it is also possible that poor executive function is caused by higher BMI, and studies indeed found that obesity-related inflammation may impair executive functioning (e.g., Bourassa \& Sbarra, 2017). Note that the current study did not observe a significant correlation between sustained attention and BMI, which is consistent with a previous study in which inhibitory control, but not sustained attention, 
was a significant predictor of children's body weight (Wirt, Schreiber, Kesztyüs, \& Steinacker, 2015).

Inconsistent with our hypothesis, the current study did not find that TL-BS variability is a significant predictor of BMI, which is not in line with previous studies in which this relationship was observed (Liu, Nederkoorn, et al., 2019; Liu, Roefs, et al., 2019; Liu et al., 2021). The current study closely replicated the dot-probe task and stimuli used in the previous studies (Liu, Roefs, et al., 2019; Liu et al., 2021), except the current study was conducted online without an eye-tracker and paired stimuli in the current food dot-probe task were presented for a shorter duration (500 ms vs $2000 \mathrm{~ms}$ ). The shorter presentation time might have reduced attention for the pictures and therefore of variability in $\mathrm{AB}$ for food.

Importantly, we noticed that participants' response latencies were faster in the food dot-probe task of the current study $\left(R T_{\text {Mean }}=411.02 \mathrm{~ms}, R T_{S D}=62.39\right)$ as compared to previous studies (for example, $R T_{\text {Mean }}=466.13 \mathrm{~ms}, R T_{S D}=102.76$ in the study of Liu, Nederkoorn, and colleague (2019)), which may also explain the inconsistency across studies. More specifically, if participants responded fast in general, it may mean that they did not pay sufficient attention to the paired picture stimuli during the experiment and only responded to the dot probe. This might have reduced (fluctuations in) AB for food. The fast response in the food dot-probe task of the current study may be caused by a less controlled online experimental setting or by a lack of eye-tracker measurement to serve as a cue that motivates participants to move their gazes.

Taken together, these experimental details may explain the lack of a significant relationship between variability of $\mathrm{AB}$ for food and $\mathrm{BMI}$ on the one hand and between variability of $\mathrm{AB}$ for food and executive control on the other hand. It seems that longer stimulus presentation durations and a well-controlled experimental setting may be necessary to find meaningful fluctuations in attention for food and make the relation between TL-BS variability for food and BMI apparent.

Of course, it is also possible that there is indeed no significant relationship between TL-BS variability for food and executive control. In a food dot-probe task, participants are merely exposed to food pictures and there are no food intake-related cues. Nowadays food and food-related stimuli are prevalent (e.g., Hall, 2018), so it is questionable whether these 
food pictures could elicit food-related inhibitory control. As we did not find a significant relation between TL-BS variability for food and BMI, the current study is unable to conclude possible mechanisms behind this relationship. Future studies are needed to further investigate whether executive control plays a role in the possible correlation between TL-BS variability for food and BMI.

\section{Conclusion}

The current study aimed to test whether executive control can explain the relationship between variability in $\mathrm{AB}$ for food and BMI. It was found that poor response inhibition (mSSRT) significantly predicted higher BMI. However, the current study did not find that TL-BS variability for food was significantly related to BMI, nor that executive control (both response inhibition and sustained attention) was significantly related to TL-BS variability for food. Results suggest that longer stimuli presentations and well-controlled experimental settings may be vital to make the relation between TL-BS variability for food and BMI apparent. In addition, more studies are needed to test if executive control can explain the relation, of course, a study needs to be done in which this relationship is apparent. 
Chapter 6

\section{Attentional Capture by Food Distractors: The Role of Perceptual Load}

Submitted as:

Liu, Y., Roefs, A., \& Nederkoorn, C. (2021). Attentional capture by food distractors: the role of perceptual load. Psychological research. 


\section{Abstract}

Food is vital for life: it is not only the main source of nutrition but also a source of reward. Previous studies found that food stimuli were more salient as compared to non-food stimuli. The perceptual load theory (Lavie, Hirst, De Fockert, \& Viding, 2004) proposed that a high perceptual load during a task reduced interference from task-irrelevant distractors because people then have less perceptual capacity left to attend these distractors. However, salient stimuli, like famous faces, appeared more resistant and even attracted attention when the perceptual load was high. This study tested the effect of a perceptual load on the attentional capture by food distractors and whether palatable foods, as opposed to non-food neutral distractors, could similarly escape the effect of a high perceptual load. To achieve our aims, in an online experiment with a within-subjects study design, a perceptual-load-plus-distractor task was conducted among 61 females. No evidence was found for the idea that palatable foods could specifically escape the effect of a high perceptual load. Instead, food distractors did not capture more attention than neutral distractors in both low and high perceptual load conditions. In addition, both food and neutral distractors, in both low and high perceptual load conditions, led to more interference as compared to a no-distractor condition. This study does therefore not support the idea that palatable foods capture attention more than neutral distractors in a high load condition. Instead, both food and non-food neutral distractors equally interfered with the task irrespective of the load condition.

Keywords: perceptual load; attentional capture; interference; palatable food; restrained eating 


\section{Introduction}

Attention can be automatically attracted by salient stimuli in our environment, for example, by stimuli that are significant for survival (Carretié, 2014), which is bottom-up attention (Connor, Egeth, \& Yantis, 2004). Food might be such a stimulus: it provides nutrients needed for survival and is also a source of reward. From an evolutionary perspective, searching for high-calorie foods is an important function of the human brain (Drewnowski, 1997). As a consequence, it is generally assumed that high-calorie palatable foods are more salient and capture more attention as compared to other non-food neutral stimuli (e.g., Davids et al., 2010; McSorley, Morriss, \& van Reekum, 2017; Nijs \& Franken, 2012). The current study aims to test if high-calorie palatable foods have this attention-grabbing power when people experience a concurrent high perceptual load task.

The empirical evidence on whether food captures more attention than non-food neutral stimuli is inconsistent. Some previous studies indeed support this idea. For example, it was found that food stimuli produced more attentional capture (Foroni, Rumiati, Coricelli, \& Ambron, 2016) as compared to non-food objects, and task-irrelevant high-calorie foods, but not low-calorie foods or non-food stimuli, captured visual attention (Cunningham \& Egeth, 2018; Motoki, Saito, Nouchi, Kawashima, \& Sugiura, 2018). However, another study revealed that participants did not direct their gaze significantly more towards food pictures than paired non-food pictures (Nummenmaa, Hietanen, Calvo, \& Hyönä, 2011). In addition, normal-weight people only directed their gaze more towards food in a fasted condition but not in a fed condition (Castellanos et al., 2009). Overall, there is no robust empirical evidence that food is more salient as compared to non-food neutral stimuli. It seems that the salient nature of food is not stable and attentional capture by food may be influenced by many factors. For example, attentional capture by food may be larger when appetitive motivation is high, caused by hunger or food craving (Berridge, 2009; Field et al., 2016; Hardman et al., 2020).

One other idea is that the potential special saliency of high-calorie foods may only become evident if the perceptual system is challenged. The perceptual load theory (Lavie, Hirst, De Fockert, \& Viding, 2004) proposed that perception has limited capacity and if the surrounding information increases beyond this limited capacity, only the most relevant information will be perceptually encoded before it is manipulated in the working memory. 
This theory implies that increasing perceptual load in a relevant task leaves no capacity to encode task-irrelevant distractors. Indeed, previous studies demonstrated that increasing the perceptual load of a task reduced distraction from task-irrelevant distractors (e.g., Forster \& Lavie, 2008a; Lavie, Lin, Zokaei, \& Thoma, 2009; Gupta, Hur, \& Lavie, 2016).

Interestingly, studies also found that meaningful and familiar distractors, like musical instruments to musicians and a famous face, still capture people's attention even if the distractor was presented with a high perceptual demand task (e.g., Lavie, Ro, \& Russell, 2003; Ro, Friggel, \& Lavie, 2009; He \& Chen, 2010). The explanation is that meaningful and familiar stimuli are salient, and therefore have stronger interference effects, and some of them are processed automatically. Therefore, the perceptual encoding is not dependent on attentional capacity (Ro et al., 2009; Thoma \& Laviem 2013). Besides, people might have a face-specific capacity to process face-related information, which is different from the capacity used to perceptually encode letter string loads (Lavie et al., 2003; Thoma \& Laviem 2013).

Similarly, from an evolutionary perspective, high-calorie palatable foods are believed to be meaningful and salient (e.g., Davids et al., 2010; McSorley et al., 2017) and may therefore also continue to capture attention if the perceptual load is high. Even though the evidence for attentional capture by high-calorie food stimuli is not consistent (e.g., Foroni et al., 2016; Motoki et al., 2018; Nummenmaa et al., 2011; Castellanos et al., 2009), previous research has not considered the effect of perceptual load on attentional capture by food and it is also not clear if high-calorie palatable foods, like famous faces, still cause interference, even when there is a high perceptual load. More specifically, perhaps food only captures more attention as compared to non-food neutral stimuli when there is not enough attention capacity to process all stimuli (high perceptual load), in which case saliency of stimuli becomes important.

The current study aimed to test if food distractors, as compared to non-food neutral distractors, cause more interference when performing the perceptual task and if this is specifically the case with a high perceptual load. It was hypothesized that with a low perceptual load, both food and non-food neutral distractors would lead to interference (longer response times and more errors) as compared to the no-distractor condition, whereas with a 
high perceptual load, the food distractor condition would lead to more interference, as compared to both no-distractor and non-food distractor conditions. The current study may provide an explanation for the inconsistent empirical results in research on $\mathrm{AB}$ for palatable foods (e.g., Foroni et al., 2016; Nummenmaa et al., 2011). That is, attentional capture by palatable foods may depend on experienced perceptual load, and the special salient nature of palatable food may only become apparent when $\mathrm{AB}$ for food is studied while participants experience a high perceptual load.

\section{Method}

The current study was conducted online and it was approved by the Ethical Committee of the Faculty of Psychology and Neuroscience, Maastricht University. All participants agreed on the informed consent, in which they were informed about the procedure, storage of data, and their right to withdraw from participation without any consequence. This study was preregistered on AsPredicted (https://aspredicted.org/mi3gm.pdf).

\section{Participants}

To eliminate potential sex and age effects on task performance (Hallam, Boswell, DeVito, \& Kober, 2016), 65 females between 18-30 years old with normal or corrected to normal vision were recruited via an online recruitment system or via poster on the Facebook. To disguise the true purpose of this study and avoid influencing participants' behavior, it was informed on the poster that this study aims to compare people's perception under different conditions. The sample size was determined by a power analysis via $\mathrm{G}^{*}$ Power, which suggested including at least 28 participants (within-subject design, medium effect size, alpha $=.05$, power $=.95$, number of repeated measures $=6$ ). We aimed to test at least 50 participants, to be able to detect smaller effects. Four participants were excluded because they made mistakes on more than $50 \%$ of high load trials. After data reduction, 61 females were included in the data analysis.

\section{Materials}

\section{Questionnaires}


Dietary Restraint. The Restraint Scale (RS) is an 11-item self-report scale that is used to assess the intention to restrict food intake (Herman \& Polivy, 1980). In the current study, Cronbach's alpha was 0.77 .

Hunger. Hunger level was measured on a 100 -mm visual analogue scale (VAS) by asking "How hungry are you right now?" with 0 indicating an absolute lack of hunger and 100 indicating extreme hunger.

Food Craving. Food craving of high-calorie food stimuli presented in the task was measured on a 100 -ms VAS by asking "How much do you crave the food above?", with 0 indicating no craving at all and 100 indicating extreme craving.

\section{Perceptual-load-plus-distractor task}

Trial procedure. The task was adapted from Forster and Lavie (2008b), and it was conducted online using Inquisit Web version 6.3.5 (Millisecond Software, Seattle, WA, USA). On each trial, first, a central fixation point was displayed on the screen for $500 \mathrm{~ms}$, and next, a set of stimuli with or without a task-irrelevant distractor appeared in the middle of the screen for $100 \mathrm{~ms}$. The set of stimuli consisted of one target letter (either letter X or letter $\mathrm{N}$ ) and five nontarget letters, spaced on a circle. The size of each letter was $0.64 \mathrm{~cm}$ and the radius of the circle was $1.68 \mathrm{~cm}$. The participant was instructed to respond to the target letter as quickly and as accurately as possible by pressing the corresponding key on the keyboard (press the $\mathrm{X}$ button for the target $\mathrm{X}$ and press the $\mathrm{N}$ button for the target $\mathrm{N}$ ).

Trial types. Three different trial types were included (see Figure 1): trials with a food distractor ( $10 \%$ of trials), trials with a neutral distractor, musical instrument $(10 \%$ of trials), and trials without a distractor ( $80 \%$ of trials). Task-irrelevant distractors were displayed in only $20 \%$ of trials to ensure attentional capture by distractors and avoid habituation (Forster \& Lavie, 2008b). Task-irrelevant distractors consisted of six full-color pictures of high caloric palatable foods and 6 full-color pictures of musical instruments, which measured $3.8 \times 3.2 \mathrm{~cm}$. Task-irrelevant distractors appeared either above or below the letter circle and this position was counterbalanced over trials. The distance between the center of the picture and the center of the screen was $4.4 \mathrm{~cm}$ and the edge of the picture to the edge of the nearest letter on the circle was $0.81 \mathrm{~cm}$. The size of stimuli was not affected by screen 
size or resolution. Most of palatable high-calorie foods and musical instrument pictures were taken from Werthmann and colleagues (2011), and the remaining two stimuli were selected from the internet. Each food picture was visually matched with one musical instrument picture as closely as possible regarding shape, color, brightness, and size.

Block types. Two different block types were included: low load blocks and high load blocks. In the low load block, the nontarget letters were five O's; in the high load block, the nontarget letters were five different angular letters (randomly selected from V, E, K, H, $\mathrm{Z}$, and F). In each block, target letter $\mathrm{N}$ and target letter $\mathrm{X}$ were displayed equally often, they could appear at each location of the circle of letters, and the position of target letters was counterbalanced over trials. Non-target letters randomly appeared at each location of the letter circle on each trial.

Task. First, one 10-trial practice block at a slow pace (in which the target stimuli were presented $1500 \mathrm{~ms}$ ) and two 10-trial practice blocks at an actual pace were presented. Subsequently, 8 critical blocks were presented, including 4 low and 4 high load blocks in the order ABBAABBA or BAABBAAB. Each block consisted of 60 trials and participants were randomly and equally assigned to one of the orders. Short audio feedback was presented with incorrect and too slow (> 2s) responses. 
Figure 1. An example of the trial types.

(a)

(b)
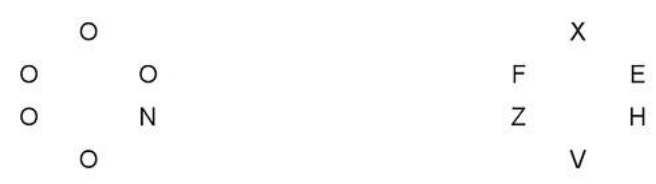

(c)

(d)

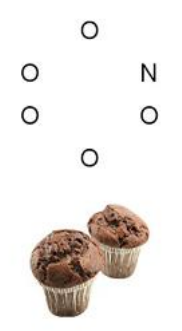

(e)

(f)

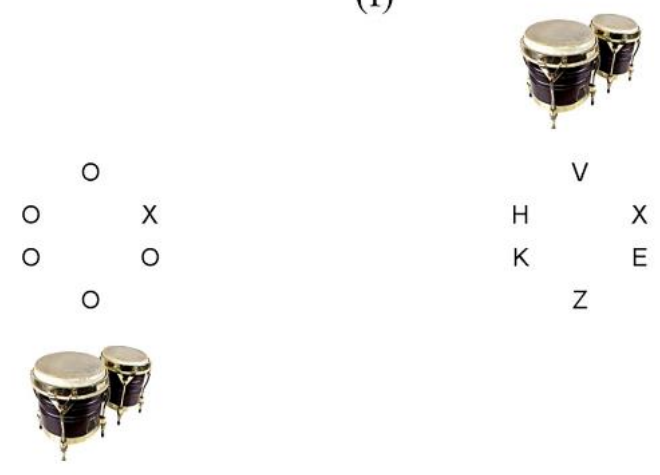

Note. (a) no distractor low load condition; (b) no distractor high load condition; (c) food distractor low load condition; (d) food distractor high load condition, (e) neutral distractor low load condition; (f) neutral distractor high load condition. 


\section{Procedure}

The study was conducted fully online. First, a link to a survey was provided in the advertisement. The survey started with an informed consent, followed by screening questions (age, sex, and vision) and a report of hunger level. Participants who were eligible for participation were automatically directed to the online task after the survey. At the beginning of the online task, the participant was instructed to complete the task in a quiet place and to wear headphones. To keep the visual angel to the displayed stimuli the same, the participant was also instructed to sit on the central axis perpendicular to the screen and to keep the distance between herself and the screen at approximately $60 \mathrm{~cm}$. It was suggested to use the length of her arm to take an approximate measure. After one block was finished, there was a small break and it was suggested to limit this break to 1 minute. After the participant finished the online task, she was directed to the final survey, which included the restraint scale and the food craving scale. All participants completed the entire procedure within 30 minutes. After the data collection of this study was finished, each participant received a debriefing email and either course credit or a 5-euro voucher, to thank her for participation.

\section{Data reduction and analysis}

Mean response times (RTs) and mean error rates were computed per load condition as measures of task performances.

\section{Date reduction}

Date reduction was conducted separately for high load and low load blocks, because RTs were significantly faster for low $(M=379.69, S D=55.61)$ than high $(M=560.25, S D=$ $120.26)$ perceptual load blocks $(t(255.47)=17.91, p<.001)$. Participants who made mistakes on more than $50 \%$ of trials in either the high load or low load condition were removed from further analyses, which led to exclusion of four participants (> 50\% mistakes in the high load condition). Trials in high load and low load conditions were excluded from further analyses if they were error responses, and after that, if they deviated more than $3 S D$ s from each participant's mean RT. As a result, $12.53 \%$ of trials in the low load condition and $24.17 \%$ of trials in the high load condition were discarded. 
In addition, mean RTs and error rates in each condition (load $\times$ distractor), and potential control variables (BMI, RS, hunger level, and food craving) were checked on outliers. If a value of a participant deviated more than 3 SDs from the corresponding mean value of all participants, the outlier value was winsorized (Wilcox, 2001) by replacing the outlier value with the nearest value within the whole sample. One outlier (3.30 SD above the mean value) for BMI, one outlier (3.05 SD above the mean value) for mean RT of food distractor in the low load condition, one outlier (3.78 SD above the mean value) for the error rate of food distractor in the low load condition, and one outlier (4.23 SD above the mean value) for the error rate of neutral distractor in the low load condition were replaced by the nearest value of the whole sample. ${ }^{7}$

\section{Analysis plan}

First, we tested whether BMI, hunger level, dietary restraint, and food craving should be added as control variables in main analyses. According to Gruijters (2016), potential confounders should be controlled for if they are significantly related to the dependent variable. As we are interested in the differential distraction from food vs neutral distractors, we first calculated the difference scores of $\mathrm{RT}\left(\mathrm{RT}_{\text {food distractor }}-\mathrm{RT}_{\text {neutral distractor }}\right)$ and error rates (Error

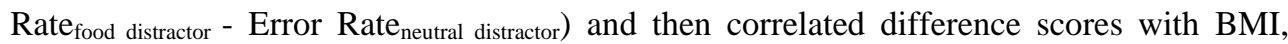
hunger level, dietary restraint, and food craving. As none of the potential control variables were significantly related to the difference scores $(r s=-.19$ to .06 , all $p s>.15)$, there was no need to control for them in main analyses.

Main hypotheses on mean RTs and error rates were tested in 2 (load: low vs high) $\times 3$ (distractor: food vs neutral vs no distractor) repeated measures ANOVAs, and Greenhouse-Geisser correction was adopted if the assumption of sphericity was violated.

\section{Results}

\section{Demographic Information}

\footnotetext{
${ }^{7}$ When no trials with correct response are excluded (following Forster \& Lavie, 2008), the results remain similar. 
Demographic information of participants is displayed in Table 1.

Table 1. Participant Characteristics

\begin{tabular}{llcl}
\hline variables & $M$ & $S D$ & Range \\
\hline RS score & 13.39 & 5.34 & $2-23$ \\
Age & 21.61 & 3.03 & $18-30$ \\
BMI & 21.93 & 3.56 & $16.02-30.93$ \\
Hunger level & 32.28 & 24.13 & $0-92$ \\
Food craving & 33.74 & 22.44 & $2-95$
\end{tabular}

Note. RS: restraint scale (Herman \& Polivy, 1980); BMI: body mass index; hunger level: the hunger level of participants (0-100 VAS); food craving: craving for high-calorie food stimuli presented in the task (0-100 VAS).

\section{Performance on the Task}

\section{Response times}

A two-way repeated measures ANOVA was run to test the effect of distractor type in high vs low load trials on RTs. In contrast to the hypothesis, there was no statistically significant interaction between distractor type and load on RTs, $F(1.62,97.25)=0.15, p=.82$, partial $\eta^{2}=.002$. However, there was a statistically significant main effect of load, $F(1,60)=$ $151.73, p<.001$, partial $\eta^{2}=0.72$. RTs were generally longer in the high than in the low load condition. The main effect of distractor type was significant as well, $F(1.63,97.54)=$ 10.47, $p<.001$, partial $\eta^{2}=0.15$. Bonferroni-corrected pairwise comparisons $(\alpha=.017)$, revealed that RTs were significantly larger with both a food distractor $(p<.01)$ and a neutral distractor $(p<.01)$, as compared to no distractor. The difference between the food and neutral distractor type was not significant $(p=1.00)$.

\section{Error rates}

A two-way repeated-measures ANOVA was run again to test the effect of distractor type in high vs low load trials on error rates. In contrast to the hypothesis, there was no statistically significant interaction between distractor type and load on error rates, $F(2,120)=$ 
$0.03, p=.997$, partial $\eta^{2}=.00005$. Again, there was a statistically significant main effect of load condition, $F(1,60)=69.45, p<.001$, partial $\eta^{2}=0.54$. Participants made significantly more errors in the high load condition than in the low load condition. There was no significant main effect of distractor type, $F(1.73,103.97)=0.72, p=.47$, partial $\eta^{2}=0.01$.

Figure 2. Mean RTs and Error Rates per distractor type and load condition.
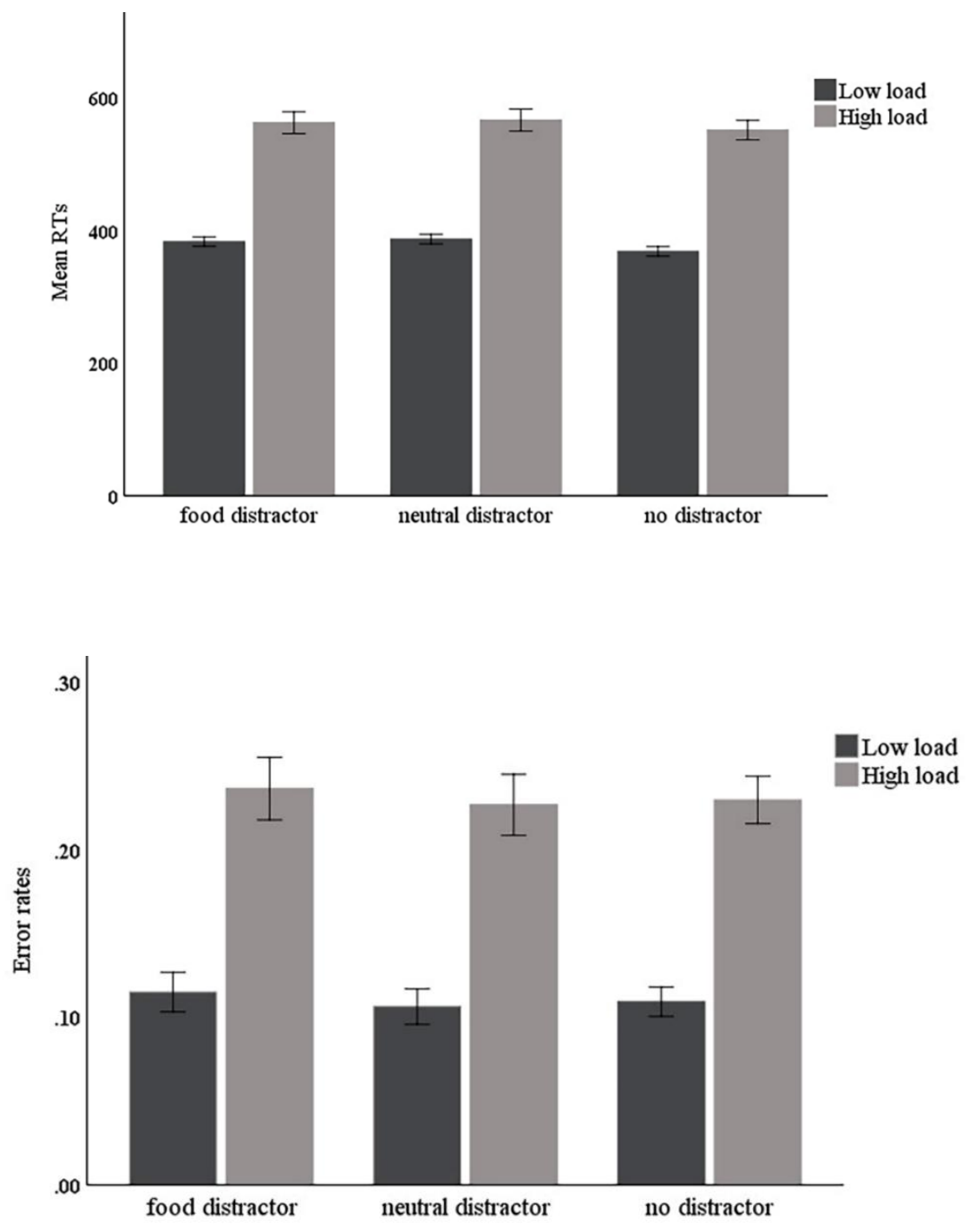

Note. Error bars reflect 1 standard error in each direction. 


\section{Discussion}

The current study aimed to test the effect of a perceptual load on attentional capture by food distractors and whether high-calorie palatable foods could draw more attention under a high perceptual load condition as compared to non-food neutral distractors. Contrary to the hypothesis, food distractors did not capture attention more than did neutral distractors, in the high load condition. Instead, we found that both the neutral and the food distractors equally interfered with task performance, in both low and high load conditions.

This study found that both food and neutral task-irrelevant distractors interfered with task performance, irrespective of perceptual load. According to previous studies (e.g., Lavie et al., 2003; Ro et al., 2009; He \& Chen, 2010), interference from meaningful and familiar distractors was found to be immune to a high perceptual load, while neutral, less relevant distractors did not interfere during a task when there was a high perceptual load. It is however doubtful that both distractor stimuli were highly salient and meaningful in the present study. In other words, it means that the results of the current study contradict the prediction from perceptual load theory (Lavie, et al., 2004; Forster \& Lavie, 2008b) and the high perceptual load failed to eliminate distraction from task-irrelevant distractors in this study. This is however not the first study that found contradictory results. For example, Lleras, Chu, and Buetti (2017) conducted six close replication experiments based on Forster and Lavie's studies (2008b), and they also found that a high perceptual load did not eliminate the attentional capture from task-irrelevant distractors in five experiments.

Contrary to our results, but consistent with the prediction from perceptual load theory (Lavie, et al., 2004; Forster \& Lavie, 2008b), a newly published study (Morris, Yeomans, \& Forster, 2020) found that the interference from both task-irrelevant food and non-food distractors disappeared in a high perceptual load condition. However, interestingly, Morris et al. (2020) also found that palatable food stimuli did not interfere more during the task than non-food stimuli in a low perceptual load condition. This study used the same perceptual-load-plus-distractor task as in the current study, to compare the interference effects from task-irrelevant palatable foods and non-food stimuli. We noted that no matter the condition, mean RTs of the current study are faster than mean RTs in Forster and Lavie's (2008b) study and Morris and colleagues' (2020) study, while participants had low error rates. 
Participants in our and other two studies were from a similar age group and therefore age cannot explain the faster responses. In addition, the high and low perceptual load used in the current study were the same as in the study of Forster and Lavie (2008b), Morris and colleagues (2020), and many others (e.g., Forster \& Lavie, 2016; Gupta et al., 2016). The present study was performed online, with less control over conditions in which participants did the task. However, faster responses and low error rates in the current study suggest that participants performed the task carefully and with full attention. It is therefore unclear why a higher load did not lead to a reduced distraction in our study and in other studies, such as Lleras et al. (2017), while in some studies it did.

The current study did not find that palatable foods were more distracting under both low and high perceptual load conditions as compared to neutral stimuli. Morris et al. (2020) also found that food did not interfere more than non-food stimuli in the low load condition. So, it is possible that palatable food distractors indeed cannot capture more attention than non-food distractors in general. When looking at the broader literature on attentional bias for food, results are very inconsistent. It was also found repeatedly that attention was not significantly biased towards high caloric foods, not even for people with obesity (e.g., Hagan, Alasmar, Exum, Chinn, \& Forbush, 2020; Werthmann Jansen, \& Roefs, 2015). Instead, some studies suggest that individuals' attentional bias for food is a dynamic process, which fluctuates over time, with people alternating between having more and less attention for food (Liu, Nederkoorn, Roefs, 2019; Liu, Roefs, Werthmann, Nederkoorn, 2019).

However, there are also several studies that found increased visual distractibility from task-irrelevant high-calorie palatable foods, as compared to neutral stimuli (Foroni et al., 2016; Cunningham \& Egeth, 2018; Motoki et al., 2018). The difference in results may be caused by methodological differences. For example, although all these tasks included a target task and a task-irrelevant distractor in each trial, measurements adopted to assess visual distractibility, difficulties in target tasks, and ways to present distractors were different. Morris et al. (2020) and our study varied task difficulty (high vs low load) and both a target and a task-irrelevant distractor were shortly and concurrently presented for $100 \mathrm{~ms}$ per trial, while other studies did not vary task difficulty and a distractor was either kept on the screen until a response (Foroni et al., 2016; Motoki et al., 2018) or randomly appeared when participants were executing the target task (Cunningham \& Egeth, 2018). Besides, Motoki et 
al. (2018) only found that participants spent more viewing time, measured by eye-tracker, on palatable food distractors than healthy food distractors, but both types of distractors equally interfered with responses to the target task. Overall, some studies found that high caloric foods can attract more attention, but results are inconsistent and seem to depend on specific methodological settings. It is possible that the western food-rich environment, in which people are frequently exposed to high-calorie palatable foods, makes palatable food not as salient as it was to our ancestors. It needs to be mentioned that the current study only included females and most of them were students, so participants were fairly homogeneous. Future studies are needed to confirm the effect of perceptual load on attentional capture by food distractors in diverse populations.

\section{Conclusion}

The current study tested if food distractors could escape a high perceptual load and interfere more in a high perceptual load task as compared to non-food neutral distractors. Contrary to our hypothesis, the results showed that high-calorie palatable foods did not lead to more interference in a high load condition as compared to non-food neutral distractors. Instead, both foods and non-food neutral stimuli equally interfered with the target task, both under high and low load conditions. The current study implies that food distractors may do not produce significantly more attentional capture as compared to non-food neutral stimuli in general and both food and non-food neutral distractors interfere with task performance irrespective of load conditions. 
Chapter 7

General Discussion 
The overarching aim of the current dissertation was to investigate the dynamic nature of attentional bias $(\mathrm{AB})$ for food. A series of studies was conducted to investigate four research questions: (1) Do people with a high BMI or dietary restraint consistently bias their attention towards food? (2) Do people with a high BMI or dietary restraint show more variability of $\mathrm{AB}$ for food? (3) Is variability of $\mathrm{AB}$ for food a reflection of conflicting food-related motivations (hedonic vs health), or can it be explained by executive control? (4) whether food is special in the sense that it can still capture attention when people perform a concurrent high perceptual load task? First, the main findings from the current dissertation will be summarized and discussed for each of these research questions. Then, a conclusion and recommendations for future directions are provided.

\section{Mean attentional bias for food}

As extensively explained in the introduction, there is no consistent empirical support for an increased $\mathrm{AB}$ for food in people with a high $\mathrm{BMI}$ or dietary restraint, as compared to people with a lower BMI or dietary restraint (e.g., Doolan, Breslin, Hanna, \& Gallagher, 2015; Hagan, Alasmar, Exum, Chinn, \& Forbush, 2020; Werthmann, Jansen, \& Roefs, 2015). To follow-up on these empirical findings, the studies described in Chapters 2-5 tested the relationship between BMI and mean $\mathrm{AB}$ for food among Dutch children, Chinese children, and female adults, and studies described in Chapters 2, 3, and 5 tested the relationship between dietary restraint and mean $\mathrm{AB}$ for food among female adults. Results consistently showed that there was no significant correlation between the mean $\mathrm{AB}$ for food and $\mathrm{BMI}$ or dietary restraint. Although these relationships were previously suggested (Hendrikse et al., 2015; Meule, Vögele, \& Kübler, 2012; Nijs \& Franken, 2012), all conducted studies in this dissertation clearly and consistently add evidence to oppose this previously theorized suggestion.

The findings from this dissertation are in line with several reviews and metaanalyses on $\mathrm{AB}$ for food, which similarly conclude that people with overweight or obesity are not characterized by more biased attention to food (e.g., Doolan et al., 2015; Hagan et al., 2020; Hardman et al., 2020; Roefs, Houben, \& Werthmann, 2015; Werthmann, Jansen, \& Roefs, 2015). This lack of a consistent AB for food can have several causes. As palatable foods can be both a source of reward and of weight and health concerns (Roefs, Fransen, \& 
Jansen, 2018), people high in BMI and dietary restraint may frequently struggle with conflicting food-related motivations, which may cause both attention towards and away from palatable foods over time. In addition, executive control, perceptual capacity, or other variables might influence the saliency of food and the extent of fluctuations in $\mathrm{AB}$ for food at that particular moment. Therefore, it seems unreasonable to suggest that $\mathrm{AB}$ for food is a stable trait-like feature in people with a high BMI or dietary restraint. As a consequence, it is relevant to investigate the dynamic nature of $\mathrm{AB}$ for food.

\section{Variability of attentional bias for food}

\section{The relationship between variability of $A B$ for food and $B M I$}

The current dissertation adopted a relatively new computing method, trial-level bias scores (TL-BS) (Zvielli, Bernstein, \& Koster, 2015), to capture the dynamic nature of AB for food in a food dot-probe task. Specifically, it examined the relation between TL-BS variability for food, the index used to quantify the extent of fluctuations in AB for food stimuli, and BMI in seven datasets which included both adults (four datasets) and children (three datasets). Results from these datasets are not entirely consistent. More specifically, it was found that overweight/obese women and obese children were characterized by significantly larger variability of $\mathrm{AB}$ for food (Chapter 2) than healthy control participants, and a significant and positive association between TL-BS variability for food and BMI appeared among Dutch children (Chapter 4) and females varying in dietary restraint (Chapter 3). In addition, to increase power, all three datasets in Chapter 2 were aggregated and a significant correlation between BMI and TL-BS variability was observed in this aggregated dataset. However, no significant correlation between TL-BS variability for food and BMI was observed in another study that included females varying in dietary restraint (Chapter 2), a study that included Chinese children (Chapter 4), and an online study that included females with normal weight, overweight, or obesity (Chapter 5). Restrained/unrestrained eaters included in Chapter 2 and participants included in Chapters 3 and 4 were predominantly healthy weight (see Table 1 for BMI range of each dataset). In short, a significant and positive association between TLBS variability for food and BMI was revealed in four of seven datasets. Although studies did not consistently show a significant association between TL-BS variability for food and BMI, some considerations suggest that there is a significant relationship between them. 
To find possible reasons for the inconsistent results, I compared the datasets of these studies. An interesting pattern was identified: the average reaction times (RTs) were faster and TL-BS variability for food was smaller for the studies that did not find a significant relationship between BMI and TL-BS variability for food, compared to the studies that did (see Table 1 for details). As it was discussed in Chapter 5, faster RTs could be due to participants paying less attention to the paired pictorial stimuli and only responding to the probe during the food dot-probe task. If participants did not attend paired pictures, this could decrease fluctuations in $\mathrm{AB}$ for food. In this case, the task might not provide an accurate measure of $\mathrm{AB}$ for food, which in turn could explain why no relation between BMI and TLBS variability for food was found.

These faster responses may have been caused by a less controlled experimental setting. More specifically, Chinese children in Chapter 4 were tested in groups (approximately 30 children per group) and the study in Chapter 5 was conducted online, whereas participants in all others were tested individually or in pairs and on-site. Although we also did not observe such a significant relationship between TL-BS variability for food and BMI in a lab-based study in Chapter 2, it should be noted that the sample size of this study was small and the BMIs of participants were in a narrow range, which possibly led to a restriction of range problem for finding a relationship between TL-BS variability and BMI. In addition, in the aggregated dataset of Chapter 2, a significant correlation between BMI and TL-BS variability was observed.

Another methodological detail, that is, the stimulus presentation duration in the food dot-probe task, might have contributed to the inconsistent results as well. More specifically, larger fluctuations in $\mathrm{AB}$ for food seem to occur predominantly in datasets with longer stimulus presentation duration (2000 ms vs $500 \mathrm{~ms}$ ) and concurrently measuring eye movements. A longer stimulus presentation duration and the presence of the eye-tracker as a reminder of the current task, might have increased attention to the paired picture stimuli and left more time for attention fluctuations. In addition, longer presentation time might have increased conflicts in food-related goals, leading to larger fluctuations.

In summary, most studies found a significant correlation between TL-BS variability for food and BMI, and significant effects were found in studies with a more controlled 
experiment setting and longer stimuli presentation duration. These findings suggest that in general, there is a positive correlation between TL-BS variability for food and BMI. It means that people with a higher BMI may have more attention fluctuations between approach and avoidance of palatable foods. In general, people with overweight or obesity have weaker executive control (e.g., Mamrot \& Hanć, 2019; Yang, Shields, Guo, \& Liu, 2018) and frequently experience conflicting motivations between food approach and weight/health concerns in the presence of food-related stimuli (Doolan et al., 2015; Field et al., 2016; Nijs $\&$ Franken, 2012). It was suggested that attentional avoidance of food-related stimuli may be a cognitive strategy to downregulate food cravings (Doolan et al., 2015). So, attention to food may shift between approach and avoidance of food-related stimuli over time. In addition, these findings also demonstrate that it is necessary to consider the role of time when assessing RT-based AB for food. Moreover, future studies investigating variability of $\mathrm{AB}$ for food should consider stimulus presentation duration carefully and conduct the study in a controlled laboratory environment. 


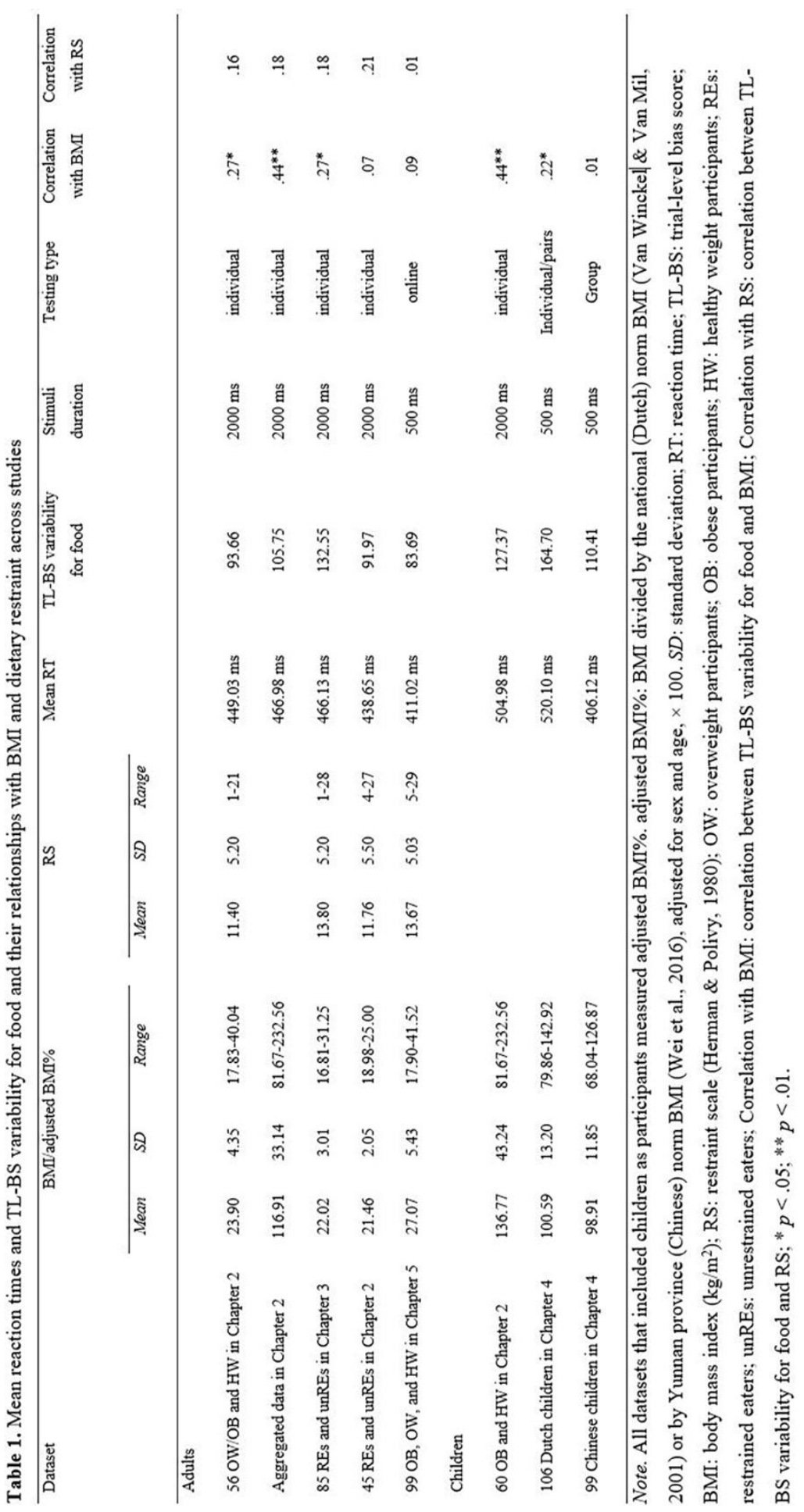




\section{The relationship between variability of $A B$ for food and dietary restraint}

It is suggested that selective attention depends on both the saliency of stimuli (bottom-up process) and momentary motivations of observers (top-down process) (Katsuki \& Constantinidis, 2014). Restrained eaters (REs) who are deliberately restricting their food intake (Herman \& Polivy, 1980) frequently experience conflict between food enjoyment and weight concerns (Stroebe, van Koningsbruggen, Papies, \& Aarts, 2013). Therefore, it was hypothesized that people high in dietary restraint should be characterized by more fluctuations between attention towards and away from food. However, studies in this dissertation consistently did not find a significant correlation between TL-BS variability for food and dietary restraint in female adults (Chapters 2, 3, and 5). Except for the study in Chapter 5, all studies that investigated the relationship between TL-BS variability for food and dietary restraint were conducted individually in a lab and the stimulus presentation time in the food dot-probe task was $2000 \mathrm{~ms}$. So, the methodological concerns mentioned before cannot explain these insignificant results. All in all, this suggests that specifically BMI, but not dietary restraint is associated with variability of $\mathrm{AB}$ for food.

This gives reasons to doubt whether TL-BS variability for food is a reflection of food-related conflicting motivations. After all, both people high in BMI and people high in dietary restraint are thought to be characterized by conflicting motivations, of wanting to eat palatable food, but also wanting to restrict food intake and have a healthy weight. It is therefore important to understand the cognitive processes that can explain the dynamic nature of $\mathrm{AB}$ for food. The studies included in Chapters 3-5 were conducted to investigate the underlying mechanism behind TL-BS variability for food. Relevant results and discussions are presented below.

\section{The underlying mechanism behind variability of attentional bias for food}

\section{The validity of TL-BS variability for food}

The biggest concern about TL-BS variability is whether it might be a byproduct of variability in general responding or biased attention in general, because a previous study using randomly generated datasets found that TL-BS variability was sensitive to fluctuations in both AB and RT (Kruijt, Field, \& Fox, 2016). To answer this question, Chapters 2 and 4 examined if the 
relationship between TL-BS variability and BMI was specific for food-related stimuli or (1) would be observed for other attractive stimuli as well, or (2) would also be observed in general responding (the standard deviation on filler trials divided by the mean RT on filler trials). Findings in Chapter 4 showed that BMI was only significantly related to the variability in food-related bias, but not to variability in nonfood-related bias. Chapter 2 did find that variability in general responding was a significant predictor of BMI in both children and adults. However, when controlling for this variability in general responding statistically, TLBS variability for food still significantly explained additional variance in BMI. Therefore, it is concluded that the significant relationship between TL-BS variability for food and BMI revealed by the conducted studies in the current dissertation is not a byproduct of variability in general response time or biased attention in general.

Studies in Chapters 2 and 3 linked indices of variability of AB for food based on reaction time (RT) to eye-movements (EMs), and tested whether TL-BS variability for food reflects overt attention towards and away from food stimuli. Results showed that TL-BS variability for food was not related to any measures of EMs-based variability of AB for food. It seems that TL-BS did not capture fluctuations in eye movements. This means that TL-BS is not a valid method to quantify overt attention. Of course, it is still possible that a different component of attention, that is, covert attention, is captured with the TL-BS method. There is still debate on the connection between covert and overt attention (Casteau \& Smith, 2020; Hunt \& Kingstone, 2003), and many previous studies did not observe a significant correlation between overt and covert AB for emotion-related stimuli (Mogg, Millar, \& Bradley, 2000; Stevens, Rist, \& Gerlach, 2011). Indeed, it is possible to shift attention without moving the eyes, and there might therefore be a distinction between overt and covert variability of $\mathrm{AB}$ for food. To learn more about this covert attention to food, future studies could investigate to which stimuli participants consciously pay more attention. Altogether, it is unclear what TL-BS measures so far. However, considering the unique variance in BMI explained by TLBS variability for food, it is worthwhile to understand what TL-BS variability reflects.

\section{Conflicting food-related motivations: hedonic vs health}

Compared to mean AB, TL-BS variability for critical stimuli (Zvielli et al., 2015) demonstrated higher split-half reliability and was more closely associated with BMI in this 
dissertation, and with some emotion-related symptoms, like depressive disorder, in several studies (Bardeen, Daniel, Hinnant, \& Orcutt, 2017; Zvielli, Vrijsen, Koster, \& Bernstein, 2016; Zvielli et al., 2015). However, the underlying mechanism behind it is still unclear. To answer this research question, the study in Chapter 3 tested the effect of context priming (hedonic, health, and neutral) on TL-BS variability for food, and whether this effect would be moderated by dietary restraint. It was assumed that hedonic priming would make the hedonic motivation more dominant, and health priming would make the health motivation more dominant. Therefore, it was hypothesized that in these two priming conditions, there would be fewer fluctuations in attention, compared to the neutral condition. However, results showed that both priming contexts and interactions between priming contexts and dietary restraint did not significantly affect TL-BS variability for food. It should be noted that the priming manipulation in Chapter 3 was not entirely successful and we only successfully induced a hedonic motivation, but not a health motivation. So, we can only conclude that participants did not consistently attend more to palatable food stimuli as compared to nonfood stimuli, and did not reduce variability in $\mathrm{AB}$, when the hedonic motivation was dominant.

In addition, results from Chapter 4 also cast doubt if conflicts in food-related motivations lead to variability of $\mathrm{AB}$ for food. Specifically, in Chapter 4, a significant correlation between TL-BS variability for food and BMI was found in Dutch children and most of Dutch children (8-12 years old) were normal weight. At least, it seems less likely Dutch children included in Chapter 4 have large conflicting food-related motivations. However, it is also possible that TL-BS variability for food reflects a more spontaneous instead of a more strategic fluctuations in food-related motivations. Specifically, weightrelated stigmatization has already widespread among children (Puhl \& Heuer, 2009) and it not only appeared in children with overweight or obesity (Pont, Puhl, Cook, \& Slusser, 2017), but also started to appear in children with normal weight (Jendrzyca \& Warschburger, 2016). It was found that experience of weight stigma in girls led to restrained eating one year later (Jendrzyca \& Warschburger, 2016). So children with (slight) overweight may also experience conflicting food-related motivations and their food-related motivations may fluctuate more spontaneously than those of adult restrained eaters. All in all, findings from 
the current dissertation raise doubt on whether TL-BS variability indeed reflects (a more strategic) food-related conflicting motivations.

\section{Executive control}

Chapters 4 and 5 tested whether executive control could explain the significant and positive association between TL-BS variability for food and BMI in Dutch and Chinese children (Chapter 4), and Dutch females (Chapter 5). Chapter 4 measured the ability of attentional control with the attention network task (ANT), and Chapter 5 measured response inhibition and sustained attention with an online stop-signal task. However, contrary to our hypothesis, we did not find that attentional control, response inhibition, or sustained attention could explain the association between TL-BS variability for food and BMI in all studies. In addition, in all studies, TL-BS variability for food was not related to the indices of executive functioning. In Chapter 5, however, the relation between TL-BS variability for food and BMI was also not replicated and doubts were raised about the validity of the use of the dot-probe task under less controlled, online circumstances. This makes the conclusion on the role of executive control less decisive.

It has been proposed that variability of $\mathrm{AB}$ for condition-relevant stimuli could be explained by executive control ever since the computing method was first proposed (Zvielli et al., 2015). However, so far, there was only one study from the emotion-related domain that tested whether attentional control could explain the relationship between TL-BS variability for critical stimuli and anxiety. Different from our findings, they found that attentional control was significantly correlated with TLBS-variability for negative words and could explain the relationship between TL-BS variability for negative words and anxiety (Clarke et al., 2020). Of course, the possibly inaccurate measurement of $\mathrm{AB}$ for food in the online dot-probe task might lead to the insignificant results of Chapter 5. However, it should be noted that food stimuli are quite different from emotion-related stimuli. As compared to emotion-related stimuli, food-related stimuli are more common, so the fluctuations in $A B$ for food stimuli might be smaller than the fluctuations in AB for emotion-related stimuli. The narrow range of variability of $\mathrm{AB}$ for food might reduce the correlation between executive control and TL-BS variability for food. More research is therefore needed before a grounded 
conclusion can be drawn whether TLBS-variability for food might reflect executive control or not.

In summary, regarding the third research question, results from this dissertation did not find clear evidence to support that either conflicting food-related motivations or executive control (attentional control, response inhibition, and sustained attention) can explain the relation between TL-BS variability for food and BMI. Although it was not the main focus of these studies, Chapter 4 found that the ability of attentional control was significantly and negatively related to adjusted BMI\% in Chinese children, and Chapter 5 found that the ability of response inhibition was significantly and negatively related to BMI in Dutch females, which is in line with previous research (e.g., Mamrot \& Hanć, 2019; Yang, Shields, Guo, \& Liu, 2018). So, executive control could be a valuable target to understand the cognitive processes of overweight and obesity.

\section{The effect of perceptual capacity on attentional capture by food}

It was revealed by previous studies and studies included in this dissertation (Chapters 2, 3, 4, and 5) that people with a high BMI and dietary restraint did not consistently show an AB for food stimuli, compared to people with a low BMI and dietary restraint (e.g., Doolan et al., 2015; Hagan et al., 2020; Werthmann et al., 2015). Therefore, it is questioned whether food is overall more salient than nonfood stimuli in general population, as food is essential for survival. The salient nature of food cues might however only be revealed in specific circumstances. The perceptual load theory (Lavie, Hirst, De Fockert, \& Viding, 2004) proposed that because of the limited capacity of perception, increasing perceptual demand of task-relevant information results in filtering of task-irrelevant information. An exception is found when the task-irrelevant distractors are salient (e.g., Lavie, Ro, \& Russell, 2003; Ro, Friggel, \& Lavie, 2009; He \& Chen, 2010). The difference between salient and less salient distractors might therefore become only apparent when there is a high perceptual load. So, if food cues are more salient, as compared to nonfood neutral distractors, food cues would still interfere with the current task performance even under high perceptual demand. Chapter 6 tested whether food distractors were more salient than nonfood neutral distractors when the task required a high or low perceptual demand via an online perceptual-load-plus-distractor task. 
Inconsistent with our hypotheses, this study found that food distractors did not distract more than nonfood neutral distractors in both the high and low load condition, and instead, both neutral and food distractors equally interfered with task performance, in both the high and low perceptual load condition. Another study (Morris, Yeomans, \& Forster, 2020), which used the same task, also found that palatable food distractors did not interfere more during the task than neutral distractors, irrespective of the load condition. In addition, previous studies (e.g., Nathan et al., 2012; Fang et al., 2019) and some of the studies included in the current dissertation (studies included in Chapter 4 and 5) found that participants did not consistently and significantly bias their attention towards high-calorie foods in a food dot-probe task, not even for people with obesity or restrained eaters. These results suggest that food stimuli do not capture more attention as compared to neutral nonfood stimuli. In the so-called western food-rich environment (e.g., Hall, 2018; Lake, 2018), people are exposed to high-calorie palatable foods almost every day. So, in a neutral or even a mild hedonic state (as suggested in Chapter 3), high-calorie palatable foods did not significantly draw increased attention at all. In conclusion, it is speculated that food is not salient as compared to other neutral nonfood stimuli. In addition, results from this study and the insignificant relationship between mean AB for food and BMI /dietary restraint observed in this dissertation challenge the idea that attention to food plays a crucial role in overweight/obesity.

\section{Conclusions}

It is concluded that (1) People with a high BMI or dietary restraint are not characterized by an enhanced AB for food. (2) Instead, people with a high BMI are characterized by increased variability of $\mathrm{AB}$ for food. (3) However, it is unclear what cognitive process(es) contribute to this increased variability of $\mathrm{AB}$ for food. We found no support that TL-BS variability for food reflects food-related conflicting motivations or that it reflects less executive control. (4) In general, food is not such a salient stimulus that it grabs more attention than neutral stimuli under a high perceptual load, and also does not grab more attention for people with a high BMI or dietary restraint. Taken together, the findings in this dissertation cast doubt on the idea that attention to food plays a crucial role in overweight and obesity. However, the significant and positive correlation between $\mathrm{BMI}$ and variability in $\mathrm{AB}$ for food may suggest a useful direction in the research of healthy food choices. Specifically, it might be 
problematic/tiring for people with overweight/obesity to experience this variability all the time, which possibly compromises healthy food choices.

\section{Future research}

Our research suggests that people with a high BMI are characterized by more variability of $\mathrm{AB}$ for food, but the underlying mechanism is still unknown. On the one hand, future research could investigate the underlying mechanism behind the relation between TL-BS variability for food and BMI, and on the other hand, researchers should also consider the limitations of measuring individuals' $\mathrm{AB}$ in a lab-based computer task. In addition, our findings suggest that in general, food stimuli do not seem particularly salient, and this implies that future studies on other explanatory factors of BMI might be more fruitful.

\section{The underlying mechanism behind TL-BS variability for food}

As it was suggested before, executive control may be a candidate mechanism to explain the significant correlation between TL-BS variability for food and BMI. Based on suggestions from the current dissertation, future studies could test this in a lab-based experiment and adopt a longer stimulus presentation duration in the food dot-probe task. Findings from the current dissertation suggest that a longer stimulus presentation duration (i.e., $2000 \mathrm{~ms}$ ) and a well-controlled experimental setting might be important to find meaningful fluctuations in attention for food and to make the relation between TL-BS variability for food and BMI apparent. In addition, it would be interesting to see the relationships between the early versus late attention components and TL-BS variability for food. A way to investigate the different attention components might be to look at Event-Related Potentials (ERP). For example, the P2 ERP (occurs approximately $200 \mathrm{~ms}$ after the stimulus presentation) is associated with early attention, the P3 ERP (occurs approximately $300 \mathrm{~ms}$ after the stimulus presentation) is associated with attentional engagement, and the late positive potential (LPP) is associated with difficulties with attention disengagement (occurs approximately $800 \mathrm{~ms}$ after the stimulus presentation) (Carretié, Martín-Loeches, Hinojosa, \& Mercado, 2001; Hajcak, MacNamara, \& Olvet, 2010). Future research could therefore link TL-BS variability for food to different stages of electrophysiological brain activities during the task.

\section{Limitations of measuring individuals' AB in a lab-based computer task}


The food dot-probe task is widely used to measure people's AB for food. This dissertation revealed that people with a high BMI are characterized by more variability of $\mathrm{AB}$ for food, and people's $\mathrm{AB}$ for food fluctuates over time. However, in a lab setting, we can only measure the fluctuations of $\mathrm{AB}$ for food over a short period, in a specific setting. It would be informative to assess individuals' AB for food at periodic intervals of a day by using an app that works on mobile phones. Especially, recent studies have shown promise for using smartphone-based eye tracking without any additional hardware (using front-facing cameras to monitor eye movements) (Huang, Li, Ngai, \& Leong, 2017; Valliappan et al., 2020). This would also benefit the ecological validity of the study. For example, it was found that social context influences eating behaviors (Higgs \& Ruddock, 2020). To investigate the influence of real-world contexts on our daily eating behavior, future studies could track both $\mathrm{AB}$ for food and the pre-testing or after-testing context, such as pre/post-testing eating episodes, with such ecological momentary assessments. In addition, we speculated before that the larger fluctuations in $\mathrm{AB}$ for food in people with a higher BMI may compromise healthy food choices. To test this hypothesis, daily food menu and smartphone-based eye tracking software can be loaded in the phone which allows us to monitor participants' food choice and fluctuations in $\mathrm{AB}$ for food. Then, we can study the relations between fluctuations in $\mathrm{AB}$ for food and food choices in a way that simulates real life food choices.

\section{Beyond building relations between $A B$ for food and trait-like features of individuals}

We live in an "obesogenic environment", in which high-calorie palatable foods are everywhere (e.g., Hall, 2018; Lake, 2018). The widely accepted idea is that people are therefore frequently exposed to high-calorie palatable foods or food-related stimuli, which might cause increased attention to food and subsequent food intake. However, it is also possible that palatable foods became less salient because of repeated exposure to food-related information. This suggestion is in line with results from previous studies (e.g., Fang et al., 2019; Nummenmaa, Hietanen, Calvo, \& Hyönä, 2011) and the results in this dissertation (Chapters 3, 5, and 6). Although the findings in this dissertation cast doubt on the idea that attention to food plays a crucial role in overweight and obesity, previous studies found that participants' total fixation duration was positively related to the amount of food eaten (Pedersen et al., 2021) and children with a longer gaze duration on food cues ate more snacks (Folkvord, Anschütz, Wiers, \& Buijzen, 2015). So, AB for food may not be a characteristic 
of obesity or dietary restraint, but instead may reflect current motivational state, as it is related to consumption. Future studies can investigate whether $A B$ for food indeed reflect momentary motivational state and which factors influence such possible relation. In addition, the current dissertation also suggests that mild health-related cues in real life might not be enough to increase health/weight concerns and influence $A B$ for food. Future studies should consider using stronger health-related cues, for example, weight or dieting-related information.

Moreover, nowadays, many people share and view appetizing pictures and culinary experiences via social media on a daily basis (Hall et al., 2018). Food is more than just a source of energy supply and except for eating enjoyment and health/weight concerns, it also conveys social meaning and connects to wellbeing (Ares et al., 2015). Future studies should therefore also focus on the social meaning of food and investigate how this aspect of food affects attention and subsequent food intake, in combination with eating enjoyment or (and) weight/health concerns. This might help unravel how and when our food environment attracts our attention and influences our eating behavior. 
Summary 
The current dissertation investigated the dynamic nature of attentional bias $(\mathrm{AB})$ for food in the background of the global obesity epidemic. Theories (Berridge, 2009; Nijs \& Franken, 2012) proposed that palatable foods automatically grab attention, and this $\mathrm{AB}$ for palatable foods was proposed to be a trait-like characteristic of both restrained eaters and people with overweight/obesity (e.g., Hendrikse et al., 2015; Meule, Vögele, \& Kübler, 2012). However, empirical evidence in this field does not consistently support this hypothesis (e.g., Hagan, Alasmar, Exum, Chinn, \& Forbush, 2020; Roefs, Houben, \& Werthmann, 2015; Werthmann, Jansen, \& Roefs, 2015). Palatable foods are both a source of reward and of weight/health concerns, which, therefore, could cause people to alternate between food approach and food avoidance when confronted with palatable foods. Conflicting motivations might therefore lead to fluctuations in attention towards and away from the food over time. This dissertation aimed to quantify and understand the fluctuations in AB for food across different groups. In addition, whether food stimuli capture attention more than neutral nonfood stimuli was also studied.

Chapter 1 discussed the inconsistent findings about the stable trait-like view of $A B$ for food and the reliabilities about the dynamic view of $\mathrm{AB}$ for food. This Chapter firstly discussed and proposed that people's AB for food is not a stable process and it might be affected by conflicting food-related motivations (eating enjoyment vs weight/health concerns), the ability of executive control, and attentional capacity. After that, methods to capture the extent of fluctuations in attention to critical stimuli were discussed. Finally, the research goals of the current dissertation were elaborated. More specifically, the first two goals of this dissertation were to investigate the mean and intra-individual fluctuations in $\mathrm{AB}$ for food, which was quantified by TL-BS variability, across different groups. The third goal was to reveal the underlying mechanism behind TL-BS variability for food. The final goal was to test whether attentional capacity affected attentional capture by task-irrelevant food stimuli.

Chapter 2 investigated the relations between TL-BS variability for food and $\mathrm{BMI} /$ dietary restraint. The TL-BS computing method was adopted to reanalyze AB data on the food dot-probe task from three published earlier studies (Werthmann et al., 2015; Werthmann et al., 2011, 2013). The target groups in these studies were overweight/obese adults, obese children, and restrained eaters, which were all compared to their respective 
control group. In addition, this Chapter also tested whether TL-BS variability could still significantly explain variance in BMI or dietary restraint after controlling for the variability in general responding (reaction times (RTs)) and in mean AB score. Moreover, we also linked the RT-based AB for food, TL-BS variability, to the eye-movements (EMs)-based AB for food, dwell time variability. The results showed that both TL-BS variability for food and the variability in general responding could significantly predict variance in BMI, but could not significantly predict variance in dietary restraint. When controlling for the variability in general responding and in mean AB score, TL-BS variability for food still predicted BMI in obese children and the aggregated dataset of the three studies. However, TL-BS variability for food was not significantly correlated with dwell time variability. Therefore, it was concluded that individuals with a higher BMI are characterized by more variability in $\mathrm{AB}$ for food and in general responding, which might be caused by weak executive control ability.

Chapter 3 tested whether TL-BS variability for food was a reflection of conflicting food-related goals (hedonic vs health). More specifically, because high-calorie foods are both a source of reward and of weight/health concerns, people might fluctuate between approach and avoidance of palatable foods, which in turn might lead to fluctuations in AB for food. This conflict between food enjoyment and weight/health concerns might be larger in people who are high in dietary restraint (Stroebe, van Koningsbruggen, Papies, \& Aarts, 2013). However, if people focus exclusively on either a hedonic or a healthy facet of food, they might show less variability in $\mathrm{AB}$ to food as compared to a neutral focus. To examine this hypothesis, this study primed female participants with a hedonic, health, and neutral context successively, and examined whether the primed context influenced AB for food. In addition, it was tested if such effects were moderated by dietary restraint. Both the mean $A B$ for food and TL-BS variability for food were measured in a food dot-probe task with an eye-tracker. However, contrary to our hypotheses, neither context nor the interaction between context and dietary restraint significantly affected measures of $A B$ for food. Instead, in line with prior studies, BMI was correlated with TL-BS variability for food. In conclusion, this study does not find any support that either mean AB for food or TL-BS variability for food depends on a primed context. However, again, this study reveals a significant relationship between TLBS variability for food instead of mean AB for food and BMI. 
Chapter 4 studied the relation between TL-BS variability for food and adjusted $\mathrm{BMI} \%$ among Dutch and Chinese children. In addition, it examined whether attentional control moderated the relationship between two of them. Moreover, whether the correlation between adjusted BMI\% and TL-BS variability was specific for food stimuli or existed for other attractive nonfood stimuli as well was also investigated. The results showed that adjusted BMI\% was only positively associated with TL-BS variability for food, and not for attractive nonfood stimuli, in Dutch children. However, this significant association did not hold in Chinese children, and attentional control did not significantly moderate the relation between TL-BS variability for food and adjusted BMI\% in both Dutch and Chinese children. Unexpectedly, Chinese children had stronger attentional control ability as compared to Dutch children, and the attentional control ability was negatively related to the adjusted BMI\% in Chinese children. The results provide some evidence that both TL-BS variability for food and attentional control are related to adjusted $\mathrm{BMI} \%$.

Chapter 5 also aimed to reveal the underlying mechanism behind TL-BS variability for food. it was hypothesized that weak executive control could cause both increased attention variability and weight gain, which might, at least partly, lead to the significant association between TL-BS variability for food and BMI. Results showed that inhibitory control, measured by a stop-signal task, indeed was significantly related to BMI. However, contrary to the result of our prior research, TL-BS variability for food was not significantly related to BMI, and executive control was not significantly related to TL-BS variability for food. In conclusion, this study does not provide any evidence that executive control can explain the positive correlation between TL-BS variability for food and BMI. However, the result indicates a relationship between poor executive control and overweight/obesity.

Chapter 6 tested whether food stimuli were special in the sense that they can still grab attention under a high perceptual load. According to the perceptual load theory (Lavie, Hirst, De Fockert, \& Viding, 2004), high perceptual demand of the current task can reduce interference from task-irrelevant distractors, however, salient cues can still capture attention even under high demand. From an evolutionary perceptive, palatable foods are assumed as salient stimuli, so we speculated that palatable food distractors, but not neutral nonfood stimuli, could still attract attention even under a high perceptual load. Under a low load, we expected that both food and neutral stimuli equally distracted participants. To test our 
hypotheses, an online perceptual-load-plus-distractor task was conducted. However, we did not find that palatable foods could capture more attention than neutral nonfood distractors in both the low and high perceptual load conditions. Instead, in both perceptual load conditions, food and nonfood neutral distractors induced more interference than a no-distractor condition. The results imply that both food and non-food neutral distractors equally interfere with the current task irrespective of the load condition.

Chapter 7 summarized and discussed the main findings from this dissertation. In addition, methodological considerations and suggestions for future research were presented. Finally, a conclusion based on the main findings from this dissertation was drawn. In brief, there was no evidence to support that an increased $\mathrm{AB}$ for food is a trait-like feature in people with a higher BMI or scoring higher on dietary restraint, whereas TL-BS variability for food was positively correlated to BMI but not to dietary restraint. As for the mechanism behind the TL-BS variability for food, we found that neither conflicting food-related motivations nor executive control (attentional control, response inhibition, and sustained attention) could explain the significant relationship between TL-BS variability for food and BMI. In addition, palatable foods did not capture attention more than neutral nonfood distractors in general. Future research could continue to investigate the underlying mechanism behind the relation between TL-BS variability for food and BMI. In addition, future research should be aimed at unraveling if and how our food environment attracts our attention and influences our eating behavior. 


\section{Valorization Addendum}




\section{Main findings}

The current dissertation aimed to investigate biased attention for food, and fluctuations in this attentional bias (AB) for food, and test if this is related to BMI or dietary restraint. In summary, the findings of this dissertation show that people with overweight/obesity and restrained eaters do not significantly bias their attention more to palatable foods as compared to people with normal weight. Higher BMI is however related to more fluctuations in attention, towards and away from food. The results of this project are relevant for understanding eating behavior and helping to establish healthy diets.

\section{Relevance}

Worldwide, over 1.9 billion adults aged 18 years and older (in 2016), over 340 million children and adolescents aged 5-19 (in 2016), and over 38 million children under the age of 5 (in 2019) are overweight or obese (World Health Organization, 2020). The prevalence of obesity nearly tripled between 1975 and 2016 and continues to rise rapidly (World Health Organization, 2020; Finkelstein et al., 2012), which has caused adverse health and economic consequences (e.g., Kyrgiou et al., 2017). A correct and clear understanding of the cognitive mechanisms driving eating behavior will benefit the development of more effective interventions targeting obesity.

In general, people know that healthy eating habits and exercising benefit a healthy weight. However, it is still difficult for many to adhere to a healthy lifestyle. We live in an obesogenic environment (e.g., Hall, 2018; Lake, 2018) in which we are exposed to aggressively marketed, advertised, and affordable palatable food stimuli. Many researchers in the food domain assumed that people with overweight/obesity have an $\mathrm{AB}$ for food-related cues, which possibly promotes overeating (e.g., Berridge, 2009; Nijs \& Franken, 2012). However, the empirical evidence about it is disappointingly inconsistent (e.g., Hagan et al., 2020; Werthmann, Jansen, \& Roefs, 2015). Our results confirm that the relation between attention for food and overweight is more complex. Attention for food fluctuates over time and these fluctuations increase with higher BMI. Building on these results, future research can focus on factors that influence the relation between food cues, attention, and overweight/obesity, with the goals to determine how our food environment influences overweight/obesity and how this can be prevented. 
In addition, our findings cast doubt on whether it is appropriate to implement research findings in applied settings too quickly. The previous studies on $\mathrm{AB}$ for addictive and emotion-related stimuli have fostered the translation from scientific research to practical application, in the form of attentional bias modification training (ABT) (Heeren, Mogoașe, Philippot, \& McNally, 2015; MacLeod \& Clarke, 2015). The food-related ABT is based on the assumptions that people with overweight/obesity have an enhanced $\mathrm{AB}$ for food stimuli and a reduction of such $\mathrm{AB}$ for food stimuli should reduce food intake (Kakoschke, Kemps, \& Tiggemann, 2014). However, our research suggests that people with a high BMI and dietary restraint do not necessarily have more biased attention to food stimuli as compared to healthy control participants, and therefore reducing $\mathrm{AB}$ for food might not be a useful clinical goal. This suggests that applying ABT to reduce food intake might be an invalid training method. So, we suggest it is important to establish consistent, reliable causal factors of obesity first and to avoid applying research findings in practice too quickly.

The current findings are not only relevant for research on eating behavior, but also for related fields of research, like addiction research. Specifically, it is assumed that AB for addictive drugs is most significant in people with a substance use disorder (Robinson \& Berridge, 1993). Addictive drugs and palatable foods are both a source of reward and of concern. These parallels suggest that our findings might be translated to addiction research and that studying the dynamic nature of $\mathrm{AB}$ for addictive drugs might be relevant and important. As far as we know, no study has investigated RT-based intra-individual fluctuations in $\mathrm{AB}$ for addictive drugs at the moment.

\section{Target groups}

The current dissertation investigated the dynamic nature of $\mathrm{AB}$ for food among females with overweight or obesity, females who are struggling with dietary restraint, Chinese and Dutch children, and females with normal weight. Therefore, the current dissertation is relevant for researchers who study individuals' eating behaviors and researchers who are interested in cultural differences in mechanisms of weight gain. This dissertation is also of interest for researchers and clinicians who study how to prevent overeating and transfer scientific research to practice. Furthermore, food is related to everyone's daily life, so people from the 
general population can also gain knowledge about the cognitive mechanisms underlying eating behavior from our research.

\section{Activities}

The findings of this dissertation contribute to the current state of knowledge. Studies included in this dissertation have been presented and discussed at several scientific conferences. Scientific articles based on the research included in this dissertation were published or will be published in peer-reviewed journals. 


\section{References}


Abarca-Gómez, L., Abdeen, Z. A., Hamid, Z. A., Abu-Rmeileh, N. M., Acosta-Cazares, B., Acuin, C., ... \& Agyemang, C. (2017). Worldwide trends in body-mass index, underweight, overweight, and obesity from 1975 to 2016: a pooled analysis of 2416 population-based measurement studies in 128. 9 million children, adolescents, and adults. The Lancet, 390(10113), 2627-2642. https://doi.org/10.1016/S0140-6736(17)32129-3

Ahern, A. L., Field, M., Yokum, S., Bohon, C., \& Stice, E. (2010). Relation of dietary restraint scores to cognitive biases and reward sensitivity. Appetite, 55(1), 61-68. https://doi.org/10.1016/j.appet.2010.04.001

Amir, I., Zvielli, A., \& Bernstein, A. (2016). (De)coupling of our eyes and our mind's eye: A dynamic process perspective on attentional bias. Emotion, 16, 978986. https://doi.org/10.1037/emo0000172

Ares, G., de Saldamando, L., Giménez, A., Claret, A., Cunha, L. M., Guerrero, L., ... \& Deliza, R. (2015). Consumers' associations with wellbeing in a food-related context: A cross-cultural study. Food Quality and preference, 40, 304-315. https://doi.org/10.1016/j.foodqual.2014.06.001

Ataya, A. F., Adams, S., Mullings, E., Cooper, R. M., Attwood, A. S., \& Munafò, M. R. (2012). Internal reliability of measures of substance-related cognitive bias. Drug and alcohol dependence, 121(1-2), 148-151. https://doi.org/10.1016/j.drugalcdep.2011.08.023

Bantin, T., Stevens, S., Gerlach, A. L., \& Hermann, C. (2016). What does the facial dotprobe task tell us about attentional processes in social anxiety? A systematic review. Journal of behavior therapy and experimental psychiatry, 50, 40-51. https://doi.org/10.1016/j.jbtep.2015.04.009

Bardeen, J. R., Daniel, T. A., Hinnant, J. B., \& Orcutt, H. K. (2017). Emotion dysregulation and threat-related attention bias variability. Motivation and Emotion, 41(3), 402409. https://doi.org/10.1007/s11031-017-9604-Z

Batterink, L., Yokum, S., \& Stice, E. (2010). Body mass correlates inversely with inhibitory control in response to food among adolescent girls: an fMRI study. Neuroimage, 52(4), 1696-1703. https://doi.org/10.1016/j.neuroimage.2010.05.059 
Bellgrove, M. A., Hester, R., \& Garavan, H. (2004). The functional neuroanatomical correlates of response variability: evidence from a response inhibition task. Neuropsychologia, 42(14), 1910-1916. https://doi.org/10.1016/j.neuropsychologia.2004.05.007

Berridge, K. C. (2009). 'Liking'and 'wanting'food rewards: brain substrates and roles in eating disorders. Physiology \& behavior, 97(5), 537-550. https://doi.org/10.1016/j.physbeh.2009.02.044

Berridge, K. C., Ho, C. Y., Richard, J. M., \& DiFeliceantonio, A. G. (2010). The tempted brain eats: pleasure and desire circuits in obesity and eating disorders. Brain research, 1350, 43-64. https://doi.org/10.1016/j.brainres.2010.04.003

Boggiano, M. M., Dorsey, J. R., Thomas, J. M., and Murdaugh, D. L. (2009). The Pavlovian power of palatable food: lessons for weight-loss adherence from a new rodent model of cue-induced overeating. Int. J. Obes. 33, 693-701. https://doi.org/10.1038/ijo.2009.57

Boswell, R. G., \& Kober, H. (2016). Food cue reactivity and craving predict eating and weight gain: a meta-analytic review. Obesity Reviews, 17, 159-177. https://doi.org/10.1111/obr.12354

Bourassa, K., \& Sbarra, D. A. (2017). Body mass and cognitive decline are indirectly associated via inflammation among aging adults. Brain, behavior, and immunity, 60, 63-70. https://doi.org/10.1016/j.bbi.2016.09.023

Bradley, B. P., Mogg, K., \& Millar, N. H. (2000). Covert and overt orienting of attention to emotional faces in anxiety. Cognition \& Emotion, 14, 789-808. https://doi.org/10.1080/02699930050156636

Braet, C., \& Crombez, G. (2003). Cognitive interference due to food cues in childhood obesity. Journal of clinical child and adolescent psychology, 32(1), 32-39. https://doi.org/10.1207/S15374424JCCP3201_04

Brownell, K. D. (2002). The environment and obesity. Eating disorders and obesity: A comprehensive handbook, 2, 433-438.

Carretié, L. (2014). Exogenous (automatic) attention to emotional stimuli: a review. Cognitive, Affective, \& Behavioral Neuroscience, 14(4), 1228-1258. https://doi.org/10.3758/s13415-014-0270-2 
Casteau, S., \& Smith, D. T. (2020). Covert attention beyond the range of eye-movements: Evidence for a dissociation between exogenous and endogenous orienting. Cortex, 122, 170-186. https://doi.org/10.1016/j.cortex.2018.11.007

Castellanos, E. H., Charboneau, E., Dietrich, M. S., Park, S., Bradley, B. P., Mogg, K., \& Cowan, R. L. (2009). Obese adults have visual attention bias for food cue images: evidence for altered reward system function. International journal of obesity, 33(9), 1063-1073. https://doi.org/10.1038/ijo.2009.138

Caudek, C., Ceccarini, F., \& Sica, C. (2017). Facial expression movement enhances the measurement of temporal dynamics of attentional bias in the dot-probe task. Behaviour Research and Therapy, 95, 58-70. https://doi.org/10.1016/j.brat.2017.05.003

Chen, S., Jia, Y., \& Woltering, S. (2018). Neural differences of inhibitory control between adolescents with obesity and their peers. International Journal of Obesity, 42(10), 1753-1761. https://doi.org/10.1038/s41366-018-0142-X

Clarke, P. J. F., Marinovic, W., Todd, J., Basanovic, J., Chen, N. T. M., \& Notebaert, L. (2020). What is attention bias variability? Examining the potential roles of attention control and response time variability in its relationship with anxiety. Behaviour Research and Therapy, 135, 103751. https://doi.org/10.1016/j.brat.2020.103751

Cohen, J. (1988). Statistical power analysis for the behavioral sciences. Hillsdale, New Jersey: Lawrence Erlbaum Associates.

Connor, C. E., Egeth, H. E., \& Yantis, S. (2004). Visual attention: bottom-up versus topdown. Current biology, 14(19), R850-R852. https://doi.org/10.1016/j.cub.2004.09.041

Cunningham, C. A., \& Egeth, H. E. (2018). The capture of attention by entirely irrelevant pictures of calorie-dense foods. Psychonomic bulletin \& review, 25(2), 586-595. https://doi.org/10.3758/s13423-017-1375-8

Damiano, S. R., Paxton, S. J., Wertheim, E. H., McLean, S. A., \& Gregg, K. J. (2015). Dietary restraint of 5 year old girls: Associations with internalization of the thin ideal and maternal, media, and peer influences. International Journal of Eating Disorders, 48(8), 1166-1169. https://doi.org/10.1002/eat.22432 
Davids, S., Lauffer, H., Thoms, K., Jagdhuhn, M., Hirschfeld, H., Domin, M., ... \& Lotze, M. (2010). Increased dorsolateral prefrontal cortex activation in obese children during observation of food stimuli. International journal of obesity, 34(1), 94-104. https://doi.org/10.1038/ijo.2009.193

Deluchi, M., Costa, F. S., Friedman, R., Goncalves, R., \& Bizarro, L. (2017). Attentional bias to unhealthy food in individuals with severe obesity and binge eating. Appetite, 108, 471-476. https://doi.org/10.1016/j.appet.2016.11.012

Diamond, A. (2013). Executive functions. Annual review of psychology, 64, 135-168. https://doi.org/10.1146/annurev-psych-113011-143750

Driver, J. (2001). A selective review of selective attention research from the past century. British Journal of Psychology, 92(1), 53-78. https://doi.org/10.1348/000712601162103

Doolan, K. J., Breslin, G., Hanna, D., \& Gallagher, A. M. (2015). Attentional bias to foodrelated visual cues: is there a role in obesity?. Proceedings of the Nutrition Society, 74(1), 37-45. https://doi.org/10.1017/S002966511400144X

Doolan, K. J., Breslin, G., Hanna, D., Murphy, K., \& Gallagher, A. M. (2014). Visual attention to food cues in obesity: An eye-tracking study. Obesity, 22(12), 25012507. https://doi.org/10.1002/oby.20884

Drewnowski, A. (1997a). Taste preferences and food intake. Annual Review of Nutrition, 17, 237-253. https://doi.org/10.1146/annurev.nutr.17.1.237

Drewnowski, A. (1997b). Why do we like fat? Journal of the American Dietetic Association, 97(7), S58-S62. https://doi.org/10.1016/S0002-8223(97)00732-3

Drobes, D. J., Oliver, J. A., Correa, J. B., \& Evans, D. E. (2019). Attentional bias and smoking. Neuroscience of Nicotine, 145-150. https://doi.org/10.1016/B978-0-12$\underline{813035-3.00018-6}$

Engle, R. W. (2018). Working memory and executive attention: A revisit. Perspectives on Psychological Science, 13(2), 190-193. https://doi.org/10.1177/1745691617720478

Eriksen, B. A., \& Eriksen, C. W. (1974). Effects of noise letters upon the identification of a target letter in a nonsearch task. Perception \& Psychophysics, 16(1), 143-149. https://doi.org/10.3758/bf03203267 
Fang, C. T., Chen, V. C. H., Ma, H. T., Chao, H. H., Ho, M. C., \& Gossop, M. (2019). Attentional Bias, “Cool” and "Hot” Executive Functions in Obese Patients: Roles of Body Mass Index, Binge Eating, and Eating Style. Journal of clinical psychopharmacology, 39(2), 145-152. https://doi.org/10.1097/JCP.0000000000001016

Favieri, F., Forte, G., \& Casagrande, M. (2019). The executive functions in overweight and obesity: A systematic review of neuropsychological cross-sectional and longitudinal studies. Frontiers in psychology, 10, 2126. https://doi.org/10.3389/fpsyg.2019.02126

Field, M., \& Cox, W. M. (2008). Attentional bias in addictive behaviors: a review of its development, causes, and consequences. Drug and alcohol dependence, 97(1-2), 1-20. https://doi.org/10.1016/j.drugalcdep.2008.03.030

Field, M., Werthmann, J., Franken, I., Hofmann, W., Hogarth, L., \& Roefs, A. (2016). The role of attentional bias in obesity and addiction. Health Psychology, 35(8), 767780. https://doi.org/10.1037/hea0000405.

Finkelstein, E. A., Khavjou, O. A., Thompson, H., Trogdon, J. G., Pan, L., Sherry, B., et al. (2012). Obesity and severe obesity forecasts through 2030. American Journal of Preventive Medicine, 42(6), 563-570.

https://doi.org/10.1016/j.amepre.2011.10.026

Folkvord, F., Anschütz, D. J., Wiers, R. W., \& Buijzen, M. (2015). The role of attentional bias in the effect of food advertising on actual food intake among children. Appetite, 84, 251-258. https://doi.org/10.1016/j.appet.2014.10.016

Forster, S., \& Lavie, N. (2008a). Attentional capture by entirely irrelevant distractors. Visual cognition, 16(2-3), 200-214. https://doi.org/10.1080/13506280701465049

Forster, S., \& Lavie, N. (2008b). Failures to ignore entirely irrelevant distractors: the role of load. Journal of Experimental Psychology: Applied, 14(1), 73-83. http://dx.doi.org/10.1037/1076-898X.14.1.73

Forster, S., \& Lavie, N. (2016). Establishing the attention-distractibility trait. Psychological science, 27(2), 203-212. https://doi.org/10.1177/0956797615617761 
Foroni, F., Rumiati, R. I., Coricelli, C., \& Ambron, E. (2016). A bait we cannot avoid: Food-induced motor distractibility. Brain and cognition, 110, 74-84. https://doi.org/10.1016/j.bandc.2015.12.003

Frankort, A., Roefs, A., Siep, N., Roebroeck, A., Havermans, R., \& Jansen, A. (2012). Reward activity in satiated overweight women is decreased during unbiased viewing but increased when imagining taste: an event-related fMRI study. International Journal of Obesity, 36(5), 627-637. http://dx.doi.org/10.1038/ijo.2011.213

Gearhardt, A. N., Treat, T. A., Hollingworth, A., \& Corbin, W. R. (2012). The relationship between eating-related individual differences and visual attention to foods high in added fat and sugar. Eating behaviors, 13(4), 371-374. https://doi.org/10.1016/j.eatbeh.2012.07.004

Graham, R., Hoover, A., Ceballos, N. A., \& Komogortsev, O. (2011). Body mass index moderates gaze orienting biases and pupil diameter to high and low calorie food images. Appetite, 56(3), 577-586. https://doi.org/10.1016/j.appet.2011.01.029

Gruijters, S. L. (2016). Baseline comparisons and covariate fishing: Bad statistical habits we should have broken yesterday. The European Health Psychologist, 18(5), 205209.

Gupta, R., Hur, Y. J., \& Lavie, N. (2016). Distracted by pleasure: Effects of positive versus negative valence on emotional capture under load. Emotion, 16(3), 328-337. http://dx.doi.org/10.1037/emo0000112

Hagan, K. E., Alasmar, A., Exum, A., Chinn, B., \& Forbush, K. T. (2020). A systematic review and meta-analysis of attentional bias toward food in individuals with overweight and obesity. Appetite, 104710. https://doi.org/10.1016/j.appet.2020.104710

Hall, K. D. (2018). Did the food environment cause the obesity epidemic?. Obesity, 26(1), 11-13. https://doi.org/10.1002/oby.22073

Hallam, J., Boswell, R. G., DeVito, E. E., \& Kober, H. (2016). Focus: sex and gender health: gender-related differences in food craving and obesity. The Yale journal of biology and medicine, 89(2), 161-173.

Hagan, K. E., Alasmar, A., Exum, A., Chinn, B., \& Forbush, K. T. (2020). A systematic review and meta-analysis of attentional bias toward food in individuals with 
overweight and obesity. Appetite, 104710.

https://doi.org/10.1016/j.appet.2020.104710

Hajcak, G., MacNamara, A., \& Olvet, D. M. (2010). Event-related potentials, emotion, and emotion regulation: an integrative review. Developmental neuropsychology, 35(2), 129-155. https://doi.org/10.1080/87565640903526504

Hardman, C. A., Jones, A., Burton, S., Duckworth, J. J., McGale, L. S., Mead, B. R., ... \& Werthmann, J. (2020). Food-related attentional bias and its associations with appetitive motivation and body weight: A systematic review and metaanalysis. Appetite, 104986. https://doi.org/10.1016/j.appet.2020.104986

He, C., Chen, A. Interference from familiar natural distractors is not eliminated by high perceptual load. Psychological Research 74, 268-276 (2010). https://doi.org/10.1007/s00426-009-0252-0

Heeren, A., Mogoașe, C., Philippot, P., \& McNally, R. J. (2015). Attention bias modification for social anxiety: a systematic review and meta-analysis. Clinical psychology review, 40, 76-90. https://doi.org/10.1016/j.cpr.2015.06.001

Hendrikse, J. J., Cachia, R. L., Kothe, E. J., McPhie, S., Skouteris, H., \& Hayden, M. J. (2015). Attentional biases for food cues in overweight and individuals with obesity: a systematic review of the literature. Obesity reviews, 16(5), 424-432. https://doi.org/10.1111/obr.12265

Herman, C. P., \& Polivy, J. (1980). Restrained eating. In A. J. Stunkard (Ed.), Obesity (pp. 209-224). Philadelphia: Saunders.

Higgs, S., \& Ruddock, H. (2020). Social influences on eating. Handbook of eating and drinking: Interdisciplinary perspectives, 277-291. https://doi.org/10.1007/978-3030-14504-0_27

Higgs, S., Spetter, M. S., Thomas, J. M., Rotshtein, P., Lee, M., Hallschmid, M., et al. (2017). Interactions between metabolic, reward and cognitive processes in appetite control: Implications for novel weight management therapies. Journal of Psychopharmacology, 31, 1460-1474. https://doi.org/10.1177/0269881117736917

Hill, J. O., \& Peters, J. C. (1998). Environmental contributions to the obesity epidemic. Science, 280, 1371-1374. https://doi.org/10.1126/science.280.5368.1371 
Hodge, B., Han, S. \& McBride, D.M. No evidence of attentional bias to food words among non-clinical female restrained eaters. Curr Psychol (2020). https://doi.org/10.1007/s12144-020-00933-8

Hollitt, S., Kemps, E., Tiggemann, M., Smeets, E., \& Mills, J. S. (2010). Components of attentional bias for food cues among restrained eaters. Appetite, 54(2), 309-313. https://doi.org/10.1016/j.appet.2009.12.005

Houben, K. (2011). Overcoming the urge to splurge: Influencing eating behavior by manipulating inhibitory control. Journal of behavior therapy and experimental psychiatry, 42(3), 384-388. https://doi.org/10.1016/j.jbtep.2011.02.008

Houben, K., Nederkoorn, C., \& Jansen, A. (2014). Eating on impulse: The relation between overweight and food-specific inhibitory control. Obesity, 22(5), E6-E8. https://doi.org/10.1002/oby.20670

Huang, M. X., Li, J., Ngai, G., \& Leong, H. V. (2017, May). Screenglint: Practical, in-situ gaze estimation on smartphones. In Proceedings of the 2017 CHI Conference on Human Factors in Computing Systems (pp. 2546-2557). https://doi.org/10.1145/3025453.3025794

Hultsch, D. F., MacDonald, S. W., \& Dixon, R. A. (2002). Variability in reaction time performance of younger and older adults. The Journals of Gerontology Series B: Psychological Sciences and Social Sciences, 57(2), P101-P115. https://doi.org/10.1093/geronb/57.2.P101

Hume, D. J., Howells, F. M., Rauch, H. L., Kroff, J., \& Lambert, E. V. (2015).

Electrophysiological indices of visual food cue-reactivity. Differences in obese, overweight and normal weight women. Appetite, 85, 126-137. https://doi.org/10.1016/j.appet.2014.11.012

Hunt, A. R., \& Kingstone, A. (2003). Covert and overt voluntary attention: linked or independent?. Cognitive Brain Research, 18(1), 102-105. https://doi.org/10.1016/j.cogbrainres.2003.08.006

Iacoviello, B. M., Wu, G., Abend, R., Murrough, J. W., Feder, A., Fruchter, E., ... \& Neumeister, A. (2014). Attention bias variability and symptoms of posttraumatic stress disorder. Journal of Traumatic Stress, 27(2), 232-239. https://doi.org/10.1002/jts.21899

Inquisit 6. (2020). Retrieved from https://www.millisecond.com. 
Jendrzyca, A., \& Warschburger, P. (2016). Weight stigma and eating behaviours in elementary school children: A prospective population-based study. Appetite, 102, 51-59. https://doi.org/10.1016/j.appet.2016.02.005

Jensen, C. D., Duraccio, K. M., Carbine, K. A., Barnett, K. A., \& Kirwan, C. B. (2017). Motivational impact of palatable food correlates with functional brain responses to food images in adolescents. Journal of pediatric psychology, 42(5), 578-587. https://doi.org/10.1093/jpepsy/jsw091

Johnson, C. L., Tobin, D. L. and Steinberg, S. L. 1989. Etiological, developmental and treatment considerations for bulimia. Journal of College Student Psychotherapy, 3(2-4): 57-73. https://doi.org/10.1300/J035v03n02_06

Kakoschke, N., Kemps, E., \& Tiggemann, M. (2014). Attentional bias modification encourages healthy eating. Eating behaviors, 15(1), 120-124. https://doi.org/10.1016/j.eatbeh.2013.11.001

Katsuki, F., \& Constantinidis, C. (2014). Bottom-Up and Top-Down Attention. The Neuroscientist, 20(5), 509-521. https://doi.org/10.1177/1073858413514136

Kelly, T., Yang, W., Chen, C. S., Reynolds, K., \& He, J. (2008). Global burden of obesity in 2005 and projections to 2030. International journal of obesity, 32(9), 14311437. https://doi.org/10.1038/ijo.2008.102

Kemps, E., \& Tiggemann, M. (2009). Attentional bias for craving-related (chocolate) food cues. Experimental and clinical psychopharmacology, 17, 425-433. https://doi.org/10.1037/a0017796

Kemps, E., Tiggemann, M., \& Hollitt, S. (2014). Biased attentional processing of food cues and modification in obese individuals. Health Psychology, 33(11), 13911401. https://doi.org/10.1037/hea0000069

Kruijt, A. W., Field, A. P., \& Fox, E. (2016). Capturing Dynamics of Biased Attention: Are New Attention Variability Measures the Way Forward? PLoS One, 11(11), e0166600. https://doi.org/10.1371/journal.pone.0166600

Kutner, M. H., Nachtsheim, C. J., Neter, J., \& Li, W. (2005). Applied linear statistical models (Vol. 5). Boston: McGraw-Hill Irwin.

Kyrgiou, M., Kalliala, I., Markozannes, G., Gunter, M.J., Paraskevaidis, E., Gabra, H., et al., 2017. Adiposity and cancer at major anatomical sites: umbrella review of the literature. Br. Med. J. 356, j477. https://doi.org/10.1136/bmj.j477 
Lake, A. A. (2018). Neighbourhood food environments: food choice, foodscapes and planning for health. Proceedings of the Nutrition Society, 77(3), 239-246. https://doi.org/10.1017/S0029665118000022

Lake, A., \& Townshend, T. (2006). Obesogenic environments: exploring the built and food environments. The Journal of the Royal society for the Promotion of Health, 126(6), 262-267. https://doi.org/10.1177/1466424006070487

Lan, X., Ponitz, C. C., Miller, K. F., Li, S., Cortina, K., Perry, M., et al (2009). Keeping their attention: Classroom practices associated with behavioral engagement in first grade mathematics classes in China and the United States. Early Childhood Research Quarterly, 24, 198-211. https://doi.org/10.1016/j.ecresq.2009.03.002

Lavagnino, L., Arnone, D., Cao, B., Soares, J. C., \& Selvaraj, S. (2016). Inhibitory control in obesity and binge eating disorder: A systematic review and meta-analysis of neurocognitive and neuroimaging studies. Neuroscience \& Biobehavioral Reviews, 68, 714-726. https://doi.org/10.1016/j.neubiorev.2016.06.041

Lavie, N., Hirst, A., De Fockert, J. W., \& Viding, E. (2004). Load theory of selective attention and cognitive control. Journal of experimental psychology: General, 133(3), 339-354. https://doi.org/10.1037/0096-3445.133.3.339

Lavie, N., Lin, Z., Zokaei, N., \& Thoma, V. (2009). The role of perceptual load in object recognition. Journal of Experimental Psychology: Human Perception and Performance, 35(5), 1346-1358. https://doi.org/10.1037/a0016454

Lavie, N., Ro, T., \& Russell, C. (2003). The role of perceptual load in processing distractor faces. Psychological science, 14(5), 510-515. https://doi.org/10.1111/1467$\underline{9280.03453}$

Lapenta, O. M., Di Sierve, K., de Macedo, E. C., Fregni, F., \& Boggio, P. S. (2014). Transcranial direct current stimulation modulates ERP-indexed inhibitory control and reduces food consumption. Appetite, 83, 42-48. https://doi.org/10.1016/j.appet.2014.08.005

Liu, Y., Nederkoorn, C., \& Roefs, A. (2019). Dynamics of attentional bias for food in Dutch and Chinese children and the role of executive control. Appetite, 143, 104421. https://doi.org/10.1016/j.appet.2019.104421 
Liu, Y., Roefs, A., \& Nederkoorn, C. (2021). Food Palatability Directs Our Eyes Across Contexts. Frontiers in psychology, 12, 1655. https://doi.org/10.3389/fpsyg.2021.664893

Liu, Y., Roefs, A., Werthmann, J., \& Nederkoorn, C. (2019). Dynamics of attentional bias for food in adults, children, and restrained eaters. Appetite, 135, 86-92. https://doi.org/10.1016/j.appet.2019.01.004

Lleras, A., Chu, H., \& Buetti, S. (2017). Can we "apply" the findings of Forster and Lavie (2008)? On the generalizability of attentional capture effects under varying levels of perceptual load. Journal of Experimental Psychology: Applied, 23(2), 158179. https://doi.org/10.1037/xap0000116

Lopez, R. B., Hofmann, W., Wagner, D. D., Kelley, W. M., \& Heatherton, T. F. (2014). Neural predictors of giving in to temptation in daily life. Psychological Science, 25, 1337-1344. https://doi.org/10.1177/0956797614531492

Lowe, C. J., Hall, P. A., \& Staines, W. R. (2014). The effects of continuous theta burst stimulation to the left dorsolateral prefrontal cortex on executive function, food cravings, and snack food consumption. Psychosomatic medicine, 76(7), 503-511. https://doi.org/10.1097/PSY.0000000000000090

MacLeod, C., Mathews, A., \& Tata, P. (1986). Attentional bias in emotional disorders. Journal of Abnormal Psychology, 95(1), 15-20. http://dx.doi.org/ 10.1037/0021$\underline{843 \times .95 .1 .15}$

Mamrot, P., \& Hanć, T. (2019). The Association of the Executive Functions with overweight and obesity indicators in children and adolescents: a literature review. Neuroscience \& Biobehavioral Reviews, 107, 59-68. https://doi.org/10.1016/j.neubiorev.2019.08.021

Martin, A. A., Davidson, T. L., \& McCrory, M. A. (2018). Deficits in episodic memory are related to uncontrolled eating in a sample of healthy adults. Appetite, 124, 33-42. https://doi.org/10.1016/j.appet.2017.05.011

Mazidi, M., Vig, K., Ranjbar, S., Ebrahimi, M.-R., \& Khatibi, A. (2019). Attentional bias and its temporal dynamics among war veterans suffering from chronic pain: Investigating the contribution of post-traumatic stress symptoms. Journal of Anxiety Disorders, 66, 102115. https://doi.org/10.1016/j.janxdis.2019.102115 
McNally, R. J. (2019). Attentional bias for threat: Crisis or opportunity?. Clinical psychology review, 69, 4-13. https://doi.org/10.1016/j.cpr.2018.05.005

McSorley, E., Morriss, J., \& van Reekum, C. M. (2017). Eye spy with my little eye: motivational relevance of visual stimuli guide eye-movements at different processing stages. Biological psychology, 123, 8-14.

https://doi.org/10.1016/j.biopsycho.2016.11.008

Medic, N., Ziauddeen, H., Ersche, K. D., Farooqi, I. S., Bullmore, E. T., Nathan, P. J., ... \& Fletcher, P. C. (2016). Increased body mass index is associated with specific regional alterations in brain structure. International journal of obesity, 40(7), 1177-1182. https://doi.org/10.1038/ijo.2016.42

Meule, A., Vögele, C., \& Kübler, A. (2012). Restrained eating is related to accelerated reaction to high caloric foods and cardiac autonomic dysregulation. Appetite, 58, 638-644. https://doi.org/10.1016/j.appet.2011.11.023

Miguet, M., Fillon, A., Khammassi, M., Masurier, J., Julian, V., Pereira, B., ... \& Thivel, D. (2018). Appetite, energy intake and food reward responses to an acute High Intensity Interval Exercise in adolescents with obesity. Physiology \& behavior, 195, 90-97. https://doi.org/10.1016/j.physbeh.2018.07.018

Mogg, K., Field, M., \& Bradley, B. P. (2005). Attentional and approach biases for smoking cues in smokers: an investigation of competing theoretical views of addiction. Psychopharmacology, 180(2), 333-341. https://doi.org/10.1007/s00213-005-2158$\underline{\mathrm{X}}$

Mogg, K., Millar, N., \& Bradley, B. P. (2000). Biases in eye movements to threatening facial expressions in generalized anxiety disorder and depressive disorder. Journal of Abnormal Psychology, 109(4), 695-704. https://doi.org/10.1037/0021$\underline{843 X .109 .4 .695}$

Morris, J., Yeomans, M. R., \& Forster, S. (2020). Testing a Load Theory Framework for Food-Related Cognition. Journal of Experimental Psychology: General. Advance online publication. http://dx.doi.org/10.1037/xge0000786

Motoki, K., Saito, T., Nouchi, R., Kawashima, R., \& Sugiura, M. (2018). Tastiness but not healthfulness captures automatic visual attention: Preliminary evidence from an eye-tracking study. Food Quality and Preference, 64, 148-153. https://doi.org/10.1016/j.foodqual.2017.09.014 
Naim, R., Abend, R., Wald, I., Eldar, S., Levi, O., Fruchter, E., . . Bar-Haim, Y. (2015). Threat-Related Attention Bias Variability and Posttraumatic Stress. American Journal of Psychiatry, 172(12), 1242-1250.

https://doi.org/10.1176/appi.ajp.2015.14121579

Nathan, P. J., O'Neill, B. V., Mogg, K., Bradley, B. P., Beaver, J., Bani, M., ... \& Dodds, C. M. (2012). The effects of the dopamine D3 receptor antagonist GSK598809 on attentional bias to palatable food cues in overweight and obese subjects. International Journal of Neuropsychopharmacology, 15(2), 149-161. https://doi.org/10.1017/S1461145711001052

Nederkoorn, C., Coelho, J. S., Guerrieri, R., Houben, K., \& Jansen, A. (2012). Specificity of the failure to inhibit responses in overweight children. Appetite, 59(2), 409-413. https://doi.org/10.1016/j.appet.2012.05.028

Nederkoorn, C., Smulders, F. T., Havermans, R. C., Roefs, A., \& Jansen, A. (2006). Impulsivity in obese women. Appetite, 47(2), 253-256. https://doi.org/10.1016/j.appet.2006.05.008

Nederkoorn, C., Van Eijs, Y., \& Jansen, A. (2004). Restrained eaters act on impulse. Personality and Individual Differences, 37(8), 1651-1658. https://doi.org/10.1016/j.paid.2004.02.020

Nijs, I. M., \& Franken, I. H. (2012). Attentional processing of food cues in overweight and obese individuals. Current obesity reports, 1(2), 106-113. https://doi.org/10.1007/s13679-012-0011-1

Nijs, I. M., Franken, I. H., \& Muris, P. (2008). Food cue-elicited brain potentials in obese and healthy-weight individuals. Eating behaviors, 9(4), 462-470. https://doi.org/10.1016/j.eatbeh.2008.07.009

Nijs, I. M., Franken, I. H., \& Muris, P. (2010). Food-related Stroop interference in obese and normal-weight individuals: Behavioral and electrophysiological indices. Eating behaviors, 11(4), 258-265. https://doi.org/10.1016/j.eatbeh.2010.07.002

Nijs, I. M., Muris, P., Euser, A. S., \& Franken, I. H. (2010). Differences in attention to food and food intake between overweight/obese and normal-weight females under conditions of hunger and satiety. Appetite, 54(2), 243-254. https://doi.org/10.1016/j.appet.2009.11.004 
Nummenmaa, L., Hietanen, J. K., Calvo, M. G., \& Hyona, J. (2011). Food catches the eye but not for everyone: a BMI-contingent attentional bias in rapid detection of nutriments. PLoS One, 6(5), e19215. https://doi.org/10.1371/journal.pone.0019215

Olsen, C. M. (2011). Natural rewards, neuroplasticity, and non-drug addictions. Neuropharmacology, 61(7), 1109-1122. https://doi.org/10.1016/j.neuropharm.2011.03.010

Overduin J, Jansen A \& Louwerse E (1995) Stroop interference and food intake. Int J Eat Disord 18, 277-285. https://doi.org/10.1002/1098-108X(199511)18:3<277::AIDEAT2260180310>3.0.CO;2-3

Papies, E. K., \& Hamstra, P. (2010). Goal priming and eating behavior: Enhancing selfregulation by environmental cues. Health Psychology, 29, 384-388. https://doi.org/10.1037/a0019877

Pedersen, H., Quist, J. S., Jensen, M. M., Clemmensen, K. K. B., Vistisen, D., Jørgensen, M. E., ... \& Finlayson, G. (2021). Investigation of eye tracking, electrodermal activity and facial expressions as biometric signatures of food reward and intake in normal weight adults. Food Quality and Preference, 93, 104248.

https://doi.org/10.1016/j.foodqual.2021.104248

Phaf, R. H., \& Kan, K. J. (2007). The automaticity of emotional Stroop: A metaanalysis. Journal of behavior therapy and experimental psychiatry, 38(2), 184199. https://doi.org/10.1016/j.jbtep.2006.10.008

Pont, S. J., Puhl, R., Cook, S. R., \& Slusser, W. (2017). Stigma experienced by children and adolescents with obesity. Pediatrics, 140(6), e20173034. https://doi.org/10.1542/peds.2017-3034

Posner, M. I., \& Petersen, S. E. (1990). The attention system of the human brain. Annual Review of Neuroscience, 13(1), 25-42. https://doi.org/10.1146/annurev.ne.13.030190.000325

Puhl, R. M., \& Heuer, C. A. (2009). The stigma of obesity: a review and update. Obesity, 17(5), 941-964. https://doi.org/10.1038/oby.2008.636

Riggs, N., Chou, C. P., Spruijt-Metz, D., \& Pentz, M. A. (2010). Executive cognitive function as a correlate and predictor of child food intake and physical 
activity. Child Neuropsychology, 16(3), 279-292.

https://doi.org/10.1080/09297041003601488

Riggs, N. R., Spruijt-Metz, D., Sakuma, K. L., Chou, C. P., \& Pentz, M. A. (2010). Executive cognitive function and food intake in children. Journal of nutrition education and behavior, 42(6), 398-403. https://doi.org/10.1016/j.jneb.2009.11.003

Ro, T., Friggel, A., \& Lavie, N. (2009). Musical expertise modulates the effects of visual perceptual load. Attention, Perception, \& Psychophysics, 71(4), 671-674. https://doi.org/10.3758/APP.71.4.671

Robinson, T. E., \& Berridge, K. C. (1993). The neural basis of drug craving: an incentivesensitization theory of addiction. Brain research reviews, 18(3), 247-291. https://doi.org/10.1016/0165-0173(93)90013-P

Roefs, A., Franssen, S., \& Jansen, A. (2018). The dynamic nature of food reward processing in the brain. Current Opinion in Clinical Nutrition \& Metabolic Care, 21(6), 444-448. https://doi.org/10.1097/MCO.0000000000000504

Roefs, A., Houben, K., \& Werthmann, J. (2015). Desire for food and the power of mind. In W. Hofmann \& L. F. Nordgren (Eds.), The psychology of desire (pp. 323-346). New York, NY: Guilford Press.

Rueda, M. R., Fan, J., McCandliss, B. D., Halparin, J. D., Gruber, D. B., Lercari, L. P., \& Posner, M. I. (2004). Development of attentional networks in childhood. Neuropsychologia, 42(8), 1029-1040. https://doi.org/10.1016/j.neuropsychologia.2003.12.012

Schmukle, S. C. (2005). Unreliability of the dot probe task. European Journal of Personality: Published for the European Association of Personality Psychology, 19(7), 595-605. https://doi.org/10.1002/per.554

Smith, E., Hay, P., Campbell, L., \& Trollor, J. N. (2011). A review of the association between obesity and cognitive function across the lifespan: implications for novel approaches to prevention and treatment. Obesity reviews, 12(9), 740-755. https://doi.org/10.1111/j.1467-789X.2011.00920.X

Stevenson, R. J., \& Francis, H. M. (2017). The hippocampus and the regulation of human food intake. Psychological Bulletin, 143(10), 1011-1032.

https://doi.org/10.1037/bul0000109 
Stice, E., Fisher, M., \& Lowe, M. R. (2004). Are dietary restraint scales valid measures of acute dietary restriction? Unobtrusive observational data suggest not. Psychological Assessment, 16, 51-59. https://doi.org/10.1037/1040-3590.16.1.51

Stice, E., Spoor, S., Ng, J., \& Zald, D. H. (2009). Relation of obesity to consummatory and anticipatory food reward. Physiology \& behavior, 97(5), 551-560. https://doi.org/10.1016/j.physbeh.2009.03.020

Stroebe, W., van Koningsbruggen, G. M., Papies, E. K., \& Aarts, H. (2013). Why most dieters fail but some succeed: A goal conflict model of eating behavior. Psychological Review, 120(1), 110-138. https://doi.org/10.1037/a0030849

Svaldi, J., Naumann, E., Trentowska, M., \& Schmitz, F. (2014). General and food-specific inhibitory deficits in binge eating disorder. International Journal of Eating Disorders, 47(5), 534-542. https://doi.org/10.1002/eat.22260

Swick, D., \& Ashley, V. (2017). Enhanced Attentional Bias Variability in Post-Traumatic Stress Disorder and its Relationship to More General Impairments in Cognitive Control. Scientific Reports, 7(1), 14559. https://doi.org/10.1038/s41598-017$\underline{15226-7}$

Tapper, K., Pothos, E. M., \& Lawrence, A. D. (2010). Feast your eyes: hunger and trait reward drive predict attentional bias for food cues. Emotion, 10, 949-954. https://doi.org/10.1037/a0020305

Tatangelo, G., McCabe, M., Mellor, D., \& Mealey, A. (2016). A systematic review of body dissatisfaction and sociocultural messages related to the body among preschool children. Body image, 18, 86-95. https://doi.org/10.1016/j.bodyim.2016.06.003

Thoma, V., \& Lavie, N. (2013). Perceptual load effects on processing distractor faces indicate face-specific capacity limits. Visual Cognition, 21(8), 1053-1076. https://doi.org/10.1080/13506285.2013.853717

Tremmel, M., Gerdtham, U. G., Nilsson, P. M., \& Saha, S. (2017). Economic burden of obesity: a systematic literature review. International journal of environmental research and public health, 14(4), 435. https://doi.org/10.3390/ijerph14040435

Valliappan, N., Dai, N., Steinberg, E., He, J., Rogers, K., Ramachandran, V., ... \& Navalpakkam, V. (2020). Accelerating eye movement research via accurate and 
affordable smartphone eye tracking. Nature communications, 11(1), 1-12. https://doi.org/10.1038/s41467-020-18360-5

van der Laan, L. N., Papies, E. K., Hooge, I. T., \& Smeets, P. A. (2017). Goal-directed visual attention drives health goal priming: An eye-tracking experiment. Health Psychology, 36(1), 82-90. https://doi.org/10.1037/hea0000410

van Hemel-Ruiter, M. E., de Jong, P. J., Ostafin, B. D., \& Wiers, R. W. (2015). Reward sensitivity, attentional bias, and executive control in early adolescent alcohol use. Addict Behav, 40, 84-90. https://doi.org/10.1016/j.addbeh.2014.09.004

Van Koningsbruggen, G. M., Stroebe, W., \& Aarts, H. (2013). Successful restrained eating and trait impulsiveness. Appetite, 60, 81-84.

https://doi.org/10.1016/j.appet.2012.09.016

Van Winckel, M., \& Van Mil, E. (2001). Wanneer is dik te dik? In C. Braet \& M. Van Winckel (Eds.), Behandelingsstrategieen Bij Kinderen Met Overgewicht (pp. 1126). Houten, the Netherlands: Bohn Stafleu Van Loghum.

Verbruggen, F., Aron, A. R., Band, G. P., Beste, C., Bissett, P. G., Brockett, A. T., ... \& Colzato, L. S. (2019). A consensus guide to capturing the ability to inhibit actions and impulsive behaviors in the stop-signal task. Elife, 8, e46323. https://doi.org/10.7554/eLife.46323.001

Verbruggen, F., Logan, G. D., \& Stevens, M. A. (2008). STOP-IT: Windows executable software for the stop-signal paradigm. Behavior research methods, 40(2), 479483. https://doi.org/10.3758/BRM.40.2.479

Wei, D., An, W., Huang, D., Chang, Li., Tan, M., Deng, S., Wei, X., \& Lv, H. (2016). Percentile reference values for anthropometric indices in multi ethnic students aged 7-18 in Yunnan. Chinese Journal of School Health, 37(3), 395-397. http://dx.doi.org/10.16835/j.cnki.1000-9817.2016.03.022

Werthmann, J., Jansen, A., \& Roefs, A. (2016). Make up your mind about food: A healthy mindset attenuates attention for high-calorie food in restrained eaters. Appetite, 105, 53-59. https://doi.org/10.1016/j.appet.2016.05.005

Werthmann, J., Jansen, A., \& Roefs, A. (2015). Worry or craving? A selective review of evidence for food-related attention biases in obese individuals, eating-disorder patients, restrained eaters and healthy samples. Proceedings of the Nutrition Society, 74(2), 99-114. https://doi.org/10.1017/S0029665114001451 
Werthmann, J., Jansen, A., Vreugdenhil, A. C. E., Nederkoorn, C., Schyns, G., \& Roefs, A. (2015). Food through the child's eye: An eye-tracking study on attentional bias for food in healthy-weight children and children with obesity. Health Psychology, 34(12), 1123-1132. http://dx.doi.org/10.1037/hea0000225

Werthmann, J., Roefs, A., Nederkoorn, C., \& Jansen, A. (2013). Desire lies in the eyes: attention bias for chocolate is related to craving and self-endorsed eating permission. Appetite, 70, 81-89. https://doi.org/10.1016/j.appet.2013.06.087

Werthmann, J., Roefs, A., Nederkoorn, C., Mogg, K., Bradley, B. P., \& Jansen, A. (2011). Can(not) take my eyes off it: Attention bias for food in overweight participants. Health Psychology, 30(5), 561-569. https://doi.org/10.1037/a0024291

Werthmann, J., Roefs, A., Nederkoorn, C., Mogg, K., Bradley, B. P., \& Jansen, A. (2013). Attention bias for food is independent of restraint in healthy weight individualsAn eye tracking study. Eating behaviors, 14(3), 397-400.

https://doi.org/10.1016/j.eatbeh.2013.06.005

Wilcox, R.R., 2001. Fundamentals of Modern Statistical Methods: Substantially Improving Power and Accuracy. Springer, New York.

Williams, J. M. G., Mathews, A., \& MacLeod, C. (1996). The emotional Stroop task and psychopathology. Psychological Bulletin, 120, 3-24. https://doi.org/10.1037/00332909.120.1.3

Wirt, T., Schreiber, A., Kesztyüs, D., \& Steinacker, J. M. (2015). Early life cognitive abilities and body weight: cross-sectional study of the association of inhibitory control, cognitive flexibility, and sustained attention with BMI percentiles in primary school children. Journal of obesity, 2015.

https://doi.org/10.1155/2015/534651

Wood, P. A. (2006). How Fat Works. London, Harvard University Press.

Wolfe, J. M. (1994). Guided search 2.0 a revised model of visual search. Psychonomic bulletin \& review, 1(2), 202-238. https://doi.org/10.3758/BF03200774

World Health Organization. (2016). Obesity and overweight. Fact Sheet 311. Retrieved 02.07.2013, 2013 from http://www.who.int/mediacentre/factsheets/fs311/en/

World Health Organization, 2020. WHO Obesity and Overweight. https://www.who.int/news-room/fact-sheets/detail/obesity-and-overweight 
Wright, S. M., \& Aronne, L. J. (2012). Causes of obesity. Abdominal Radiology, 37(5), 730-732. https://doi.org/10.1007/s00261-012-9862-X

Yang, Y., Shields, G. S., Guo, C., \& Liu, Y. (2018). Executive function performance in obesity and overweight individuals: A meta-analysis and review. Neuroscience \& Biobehavioral Reviews, 84, 225-244.

https://doi.org/10.1016/j.neubiorev.2017.11.020

Yokum, S., Ng, J., \& Stice, E. (2011). Attentional bias to food images associated with elevated weight and future weight gain: an fMRI study. Obesity, 19(9), 17751783. https://doi.org/10.1038/oby.2011.168

Zvielli, A., Bernstein, A., \& Koster, E. H. (2015). Temporal dynamics of attentional bias. Clinical Psychological Science, 3(5), 772-788. https://doi.org/10.1177/2167702614551572

Zvielli, A., Vrijsen, J. N., Koster, E. H. W., \& Bernstein, A. (2016). Attentional bias temporal dynamics in remitted depression. Journal of Abnormal Psychology, 125(6), 768-776. https://doi.org/10.1037/abn0000190 
Acknowledge 
Time flies quickly. It feels like just yesterday that I was landing in Amsterdam full of curiousness and started my wonderful days in Maastricht. Time flies over us, but leaves lots of treasures and good memories for me. During four years of my Ph.D. period, of course, there were some hard times, but the most vivid and deep memories of my Ph.D. period are fun and positive. These beautiful memories were made by a lot of great people, whom I would like to thanks in this way.

First of all, I would like to thank my promoters. Sjaan, my super-supervisor! You helped shape my professional and personal life. I really want to let you know how much I appreciate you for being my promoter. I still remember the day we met in the Randwyck train station and your smile cleared up my stress, worries, and tiredness. A good start! You are always so positive, enthusiastic, kind, smart, and inspiring. You were deeply involved in all phases of my Ph.D. project and gave me support all the time. To be honest, I didn't feel confident when I just start my Ph.D., but I was gaining more and more confidence with your encouragement. Right now, I am not 100 percent confident, but I know that I am improving and with less fear to continue. Thanks for all these improvements that you brought to me. For me, on one hand, you are like my family to make me feel safe and comfortable, and on the other hand, you are like my super-hero that I learned a lot from you. Your excellent characters will continue to inspire and encourage me.

Anne, my second promoter, many thanks for your supervision and all the support that you gave to me during my Ph.D. Four years ago, I read an article written by you, and your idea was interested me, and then we got contacted by email. With the help from you, Sjaan, and Anita, we successfully got the funding and I had the chance to work with all of these great people. That was kind of just fate right there and can't imagine what my life would lose if I had not read your article. You are so efficient and well-organized in work. Your creative and critical thinking always inspired me, which provided lots of new insight into my research. I learned a lot from you, like scientific thinking, academic writing, and stay positive in research. I also know how to be a successful research and a good mother from you. Many thanks for everything and I am really lucky and proud to have you and Sjaan to be my promoters. 
Then a big thanks to all members and ex-members of the eat group: Anita, Anne, Carolien, Ghislaine, Jessica, Katrijn, Lotte, Sandra, Sjaan, Alberto, Dãrta, Elien, Eric, Hanna, Kamilah, Laurens, Michelle, Pimpini, Sarah, Sieske, Stephanie, Yi, Anouk, Bart, Fania, Karolien, Iris, Remco, and Vanessa. Thank you for all the feedback and fun during our Eat meets, Brain days, drinks, or just in between. I'm proud and glad to be part of such a group of passionate researchers. You are all very nice people.

Jessie, you are always nice and warm. Whenever I was in trouble, you were always trying to help. Thanks for your care and help during my Ph.D. period. Daniela, Pula,

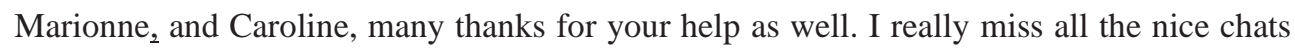
we had before.

To the Corona and assessment committee: Prof. dr. Madelon Peters, Prof. dr. Peter Muris, Dr. Nienke Jonker, Prof. dr. Ingmar Franken, Prof. dr. Henry Otgaar, Dr. Katrijn Houben, and Dr. Pauline Dibbets. Thank you for reading and assessing my dissertation and for participating in my corona.

To my roommates and friends. I'm so glad you guys were there! Martijn, Leo, Michelle, and Laurens, thanks for all the fun and help you brought to me. I already miss our days in the office. Martijn, my first roommate, thanks for letting me know lots of Dutch customs. Leo, you are so warm-hearted and you always provided your hands when I need help. Many thanks for your help and for sharing your authentic pasta recipes with us. I was so happy to get Michelle to be my roommate since I had wished that I can have a girl roommate. Thank you for all the nice conversations with you and thank you for bringing more fun to our office! Unfortunately, due to the Covid-19 regulation, I cannot meet and chat with my new roommate, Laurens, in the office, but we had several nice chats in the eat group lunch and via emails. Hope you like our clean and beautiful office.

Except for gaining knowledge in the Netherlands, I also gained a lot of friendships. I am very happy to meet you guys in Maastricht and I will cherish our friendship forever.

Sarah, my dear friend, we knew each other in the retreat of our department. We shared the room and talked a lot during these two days. Then you invited me to your home and cooked me a nice dinner... You are always so kind and warm-hearted. You helped me a lot with my eye-tracking study. During my Ph.D., we had lots of beautiful memories, and 
smiles are always there when I look back to the times together with you. I am so lucky to have you in Maastricht.

Kamila, I am so glad to meet you in our eat group. You are really kind and warm. I enjoyed a lot chatting with you in the office and our social activities. Many thanks to the good memories that you brought to me during my $\mathrm{Ph}$.D. I will definitely miss you so much in China.

My dear dance crew friends, Daniela, Angelina, Nhan, Amir, Arjun, Egle, Gintare, and Mueid, miss a lot the joyful dance party nights we had. It is really happy to dance and chat with you guys.

Riki, I am so lucky to know you in my dark times. Thanks for all the joyfulness and help that you brought to me. I am more committed to myself because of you. Hope you can get what you want.

俭勤师姐, 十分感谢你对我的帮助和鼓励, 从你身上我学到了很多, 你的优 秀一直激励着我, 祝福你事业生活双丰收!

我最好的同事和朋友, 怡, 你就像是我的定心针, 每次我有问题找聪明的吴 怡商量准没错。真的很开心和你探索马城的美食, 也很开心从你那里收获了各种知 识。有的时候看着你就像是看着我, 真的是神奇的相遇啊, 祝福我的怡可以找到她 的幸福! 期待和你在东北的再次相遇, 你还要给我买云腿月饼, 锅包肉, 大拌 菜.......

胨潼, 我在荷兰的第一个好朋友, 同时也是第一个室友。是你的温柔和帮助让 我在抵达马城, 面对空无一物的房间时没有恐惧和不安。也是你的鼓励和陪伴让我 在初来荷兰的几个月感到无比的温暖。你的善良, 勤奋, 和勇敢一直鼓励着我。很 开心你在荷兰收获了自己的幸福, 祝福你和 Mattias 幸福美满!

我亲爱的倩, 你真的是我在荷兰的意外惊喜, 没有想到在荷兰可以遇到和自 已如此心灵契合的好朋友。和你一起笑过, 疯过, 哭过, 未来我们可能不会经常见 面, 但是我知道我们依旧会是最好的朋友! 祝福我的倩心想事成, 幸福快乐! 记得 以后要约我出去玩奥。 
善良温柔的伶伶, 每次和你仰天, 心里都会变的十分平静美好。你就像有魔 力一样，总会让人内心温暖明亮。祝福你和礼川生活和事业都甜如蜜。

曼莉, 真的很开心认识你。虽然我们都不是话多的人，但是我们都知道，只 需要一向话, 彼此都会出现。谢谢你一直以来带给我的小确幸，祝福你可以依旧自 由畅快的邀游在这天地中。

最后还有盛华师兄，徐青师姐，龚英师兄，李莉，雅文，曹建花，感谢你们 给我带来的所有美好回忆，祝福你们都能心想事成，开心快乐！

My sweet and strong family, thank you for always being there with all your love and thanks for all the life thoughts that you shared to me.

我亲爱的外公, 外婆, 妈妈, 感谢你们给我创造的积极, 单纯, 自由和充满 爱的家庭环境。虽然你们的话不多, 但是你们总是在默默的付出。是你们让我拥有 了积极坚韧的人生态度, 让我学会了乐观, 无畏, 珍惜, 和尊重。而这些是我人生 中最大的财富, 它们带着你们的爱陪伴我走过了无数的岁月, 让我收获了无数的幸 福, 我知道它们还会陪伴我继续走下去。还有我的弟弟, 感谢你的陪伴, 正是因为 有你在我身边, 我的过去和未来才会不这么孤单, 希望你可以一直简单, 开心, 和 幸福! 
Curriculum Vitae 
Yu Liu (刘豫) was born on 04 September 1991 in Shangqiu City, China. After finishing high school in Shangqiu City, she received her Bachelor's education in Xinyang Normal University with a major in Applied Psychology in 2010. Subsequently, she completed a research master in Developmental and Educational Psychology at Southwest University (2014-2017). During her master, she worked on two online intervention projects targeting to reduce Chinese females' negative body image (Chinese version of the Ebody) and unhealthy eating behaviors, under the supervision of Prof. Hong Chen. From 2017 to 2021, she started her Ph.D. program in the research group: Eating Disorders and Obesity at the Department of Clinical Psychological Science at Maastricht University, supported by the Chinese Scholarship Council (CSC). During her Ph.D., she was supervised by Prof. Chantal Nederkoorn and Prof. Anne Roefs working on a project regarding the dynamic nature of attentional bias for food. 


\section{Publications}

Liu, Y., Roefs, A., \& Nederkoorn, C. (2021). Food Palatability Directs Our Eyes Across Contexts. Frontiers in Psychology, 12, 1655. https://doi.org/10.3389/fpsyg.2021.664893

Liu, Y., Nederkoorn, C., \& Roefs, A. (2019). Dynamics of attentional bias for food in Dutch and Chinese children and the role of executive control. Appetite, 143, 104421. https://doi.org/10.1016/j.appet.2019.104421

Liu, Y., Roefs, A., Werthmann, J., \& Nederkoorn, C. (2019). Dynamics of attentional bias for food in adults, children, and restrained eaters. Appetite, 135, 86-92. https://doi.org/10.1016/j.appet.2019.01.004

\section{Manuscripts under Review}

Liu, Y., Roefs, A., \& Nederkoorn, C. (under review). Attentional capture from food distractors: the role of perceptual load.

Liu, Y., Roefs, A., \& Nederkoorn, C. (under review). Fluctuations in attentional bias for food and the role of executive control.

\section{Presentations at (inter)national conferences}

Liu, Y., Roefs, A., \& Nederkoorn, C. (2021, April). Food Palatability Directs Our Eyes Across Contexts. Poster presentation at the British Feeding and Drinking Group (BFDG) 45th Annual Meeting, Online Event.

Liu, Y., Nederkoorn, C., \& Roefs, A. (2019, April). Dynamics of attentional bias for food in Dutch and Chinese children and the role of executive control. Oral presentation at the British Feeding and Drinking Group (BFDG) 43th Annual Meeting, Swansea, United Kingdom.

Liu, Y., Roefs, A., Werthmann, J., \& Nederkoorn, C. (2018, April), Dynamics of attentional bias for food in adults, children, and restrained eaters. Poster presentation at the 
Research Day of the faculty of Psychology and Neuroscience (FPN), Maastricht, the Netherlands. 